\title{
THE COMPARISON OF RAT AND HUMAN INTESTINAL AND HEPATIC GLUCURONIDATION OF ENTEROLACTONE DERIVED FROM FLAXSEED LIGNANS
}

\author{
A Thesis \\ Submitted to the College of Graduate Studies and Research \\ In partial Fulfillment of the Requirements \\ For the Degree of Master of Science \\ In the College of Pharmacy and Nutrition \\ University of Saskatchewan \\ Saskatoon
}

\section{By}

Chaojie Lin

(C) Copyright Chaojie Lin, November 2012. All rights reserved. 


\section{PERMISSION TO USE}

In presenting this thesis in partial fulfillment of the requirements for a Postgraduate degree from the University of Saskatchewan, I agree that the Libraries of this University may make it freely available for inspection. I further agree that permission for copying of this thesis in any manner, in whole or in part, for scholarly purposes may be granted by the professor or professors who supervised my thesis work or, in their absence, by the Head of the Department or the Dean of the College in which my thesis work was done. It is understood that any copying or publication or use of this thesis or parts thereof for financial gain shall not be allowed without my written permission. It is also understood that due recognition shall be given to me and to the University of Saskatchewan in any scholarly use which may be made of any material in my thesis.

Requests for permission to copy or to make other uses of materials in this thesis in whole or part should be addressed to:

Dean of the College of Pharmacy and Nutrition

110 Science Place

University of Saskatchewan

Saskatoon, Saskatchewan, S7N 5C9

Canada 


\begin{abstract}
The mammalian lignan, enterolactone (EL), is a gut microbe metabolite of plant lignan secoisolariciresinol diglucoside (SDG), which is most abundant in flaxseed. Numerous epidemiological, experimental and clinical studies suggest the protective effects of EL against various chronic diseases such as cancer, cardiovascular disease, and inflammation. However, EL's oral bioavailability is low and highly variable due to extensive first-pass metabolism, especially glucuronidation, which results in the large amount of glucuronide metabolites but low levels of free EL in human plasma. Hepatocytes and enterocytes express UDP-glucuronosyltransferases (UGT), the enzymes responsible for the conjugation of glucuronic acid to EL. To better understand the contribution of liver and intestine to the first-pass glucuronidation, I conducted an in vitro enzyme kinetic analysis of EL glucuronidation using hepatic and intestinal microsomal fractions from both human and rat. An intrinsic clearance $\left(\mathrm{CL}_{\text {int }}\right)$ value was calculated using the substrate depletion approach. In addition to monitoring substrate depletion, high-pressure liquid chromatography (HPLC) analysis allowed detection of EL glucuronides, which were further substantiated by LC-MS. EL monoglucuronide was identified in rat and human intestinal and liver microsomes. Enzyme kinetic studies indicated the extent of hepatic microsomal glucuronidation exceeded intestinal glucuronidation in both human and rat, while the human liver $\mathrm{CL}_{\text {int }}$ value was slightly higher than that of rat liver. The $\mathrm{CL}_{\text {int }}$ value generated in human intestinal microsomes was only one third of the value of human liver, whereas, the $\mathrm{CL}_{\text {int }}$ of rat jejunum or colon was one-twentieth of rat liver, suggesting the human intestine makes a greater contribution to EL glucuronidation than rat intestine. These results suggest that both liver and intestine contribute to EL glucuronidation and the human intestine may exert a greater influence on the first-pass glucuronidation of EL than rat intestine, thereby significantly decreasing EL's oral bioavailability. The rat might underestimate the extent of intestinal metabolism of EL relative to human.
\end{abstract}




\section{ACKNOWLEDGEMENT}

During my two and half year's study in Canada, I benefit so much from my supervisors, committee members, my lab mates and friends here. I would sincerely thank my supervisors Dr. Jane Alcorn and Dr. Ed Krol for their academic guidance, generous support, kind understanding and trust. I'm really grateful of Dr. Alcorn's guidance during my writing process; her comments, patient support and encouragement made me become an effective writer and researcher. I would like to thank my committee members, Dr. Andrew Olkowski and Dr. Fred Remillard for their helpful suggestions for my research work. The difficult questions asked by Dr. Andrew Olkowski before I submit my thesis challenged me, but he helped me to obtain a more clear idea of my research work and to improve my skills of answering questions. I also want to thank my external reader Dr. Lynn Weber from Department of Veterinary Biomedical Sciences for your valuable time and comments for my defense and thesis.

Sincerely thank Deborah Michel, Jennifer Billinsky, and Joshua Buse for their help on mass spectrometry during my research study. I also want to thank my lab mates Sabia Maini, Isaac Asiamah, Yunyun Di, Uma Manthena and Erica Ling for their assistance on my experimental work.

Special thanks are given to my dear friend Xue Yao and my parents for their generous support, encouragement, love and care during my master program.

I also greatly appreciate the technical help from Dorota Rogowski and Erling Madsen.

Lastly, I'm grateful of all my friends for their kindness and help during my stay in Canada.

Thanks the scholarships from University of Saskatchewan and Beijing Institute of Technology, and also the research funding from Natural Sciences and Engineering Research Council (NSERC). 


\section{TABLE OF CONTENTS}

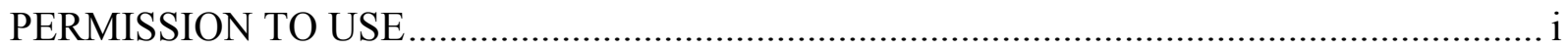

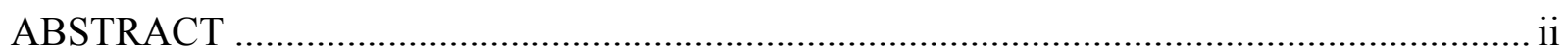

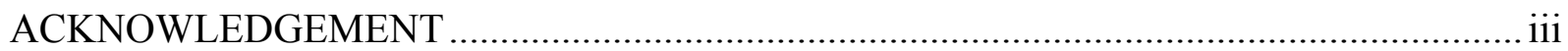

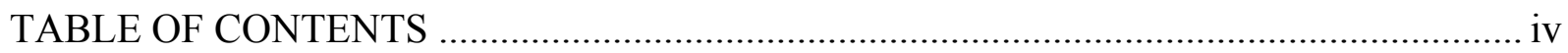

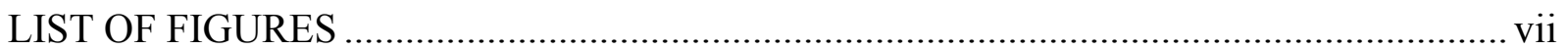

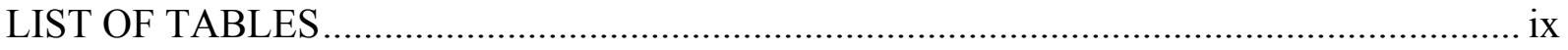

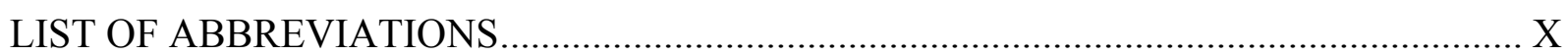

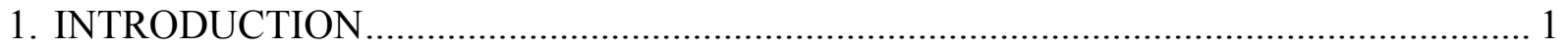

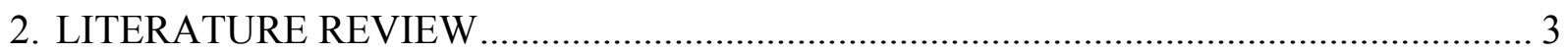

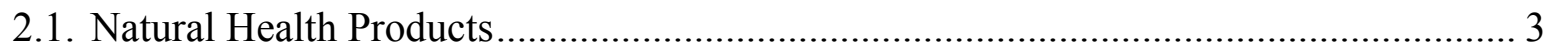

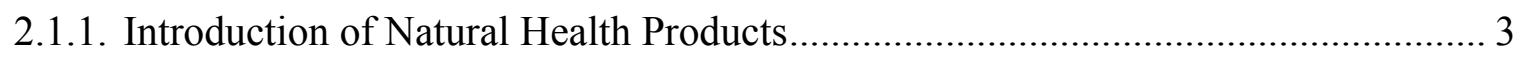

2.1.1.1. Regulation of Natural Health Products ............................................................ 3

2.1.2. Benefits and Risks of Natural Health Products ................................................... 4

2.1.2.1. Natural Health Product-drug interactions .................................................... 4

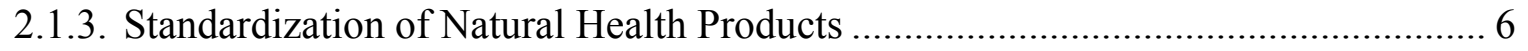

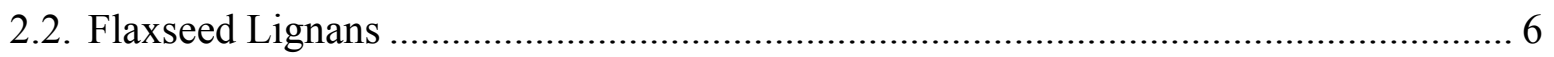

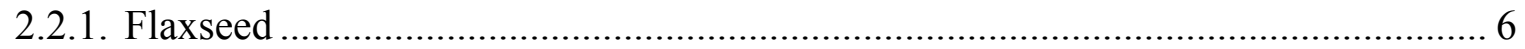

2.2.1.1. The status of flaxseed and lignans as Natural Health Product in Canada........ 7

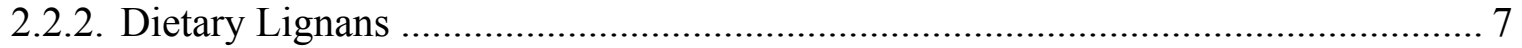

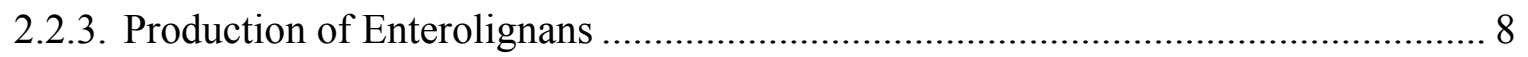

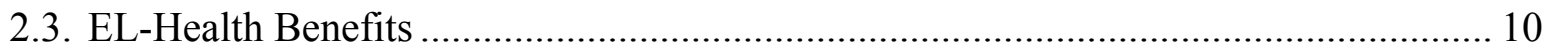

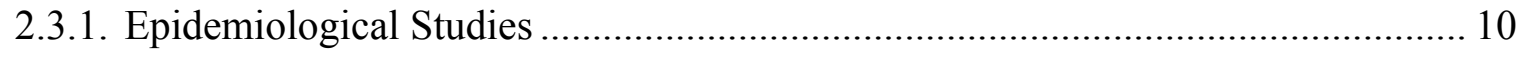

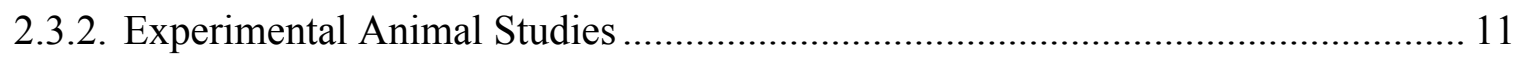

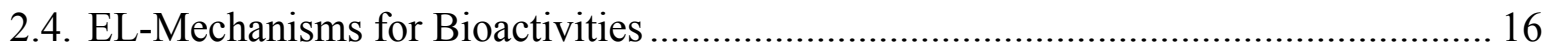

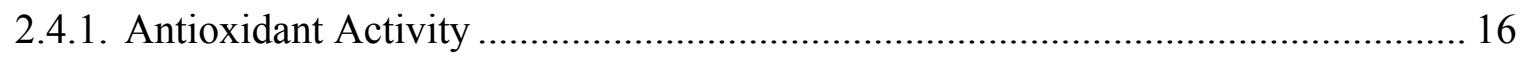

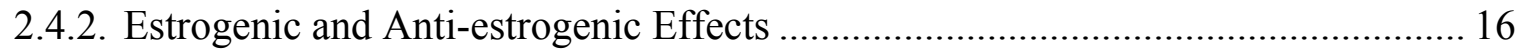

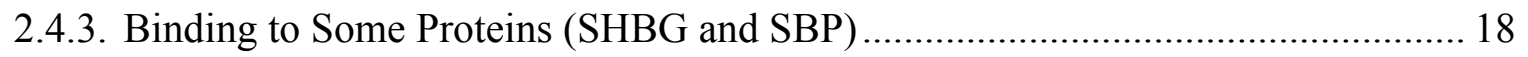

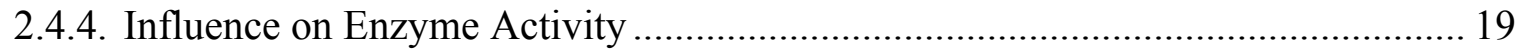


2.5. Pharmacokinetics of Plant and Mammalian lignans

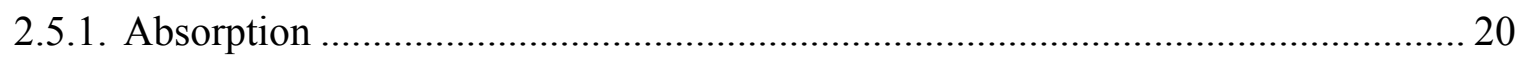

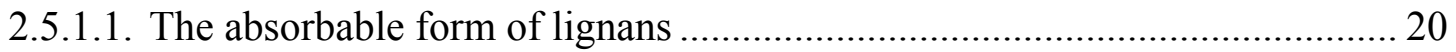

2.5.1.2. Pharmacokinetic parameters of lignans ..................................................... 21

2.5.1.3. Conversion of lignans in human gastrointestinal tract................................ 22

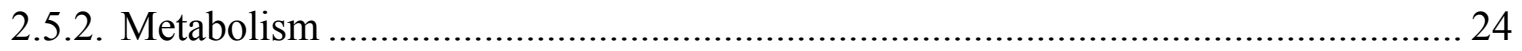

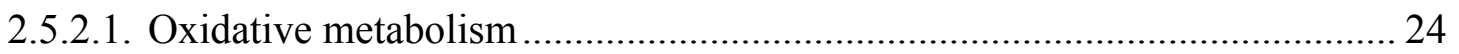

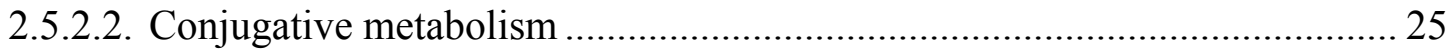

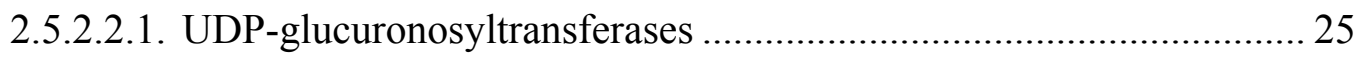

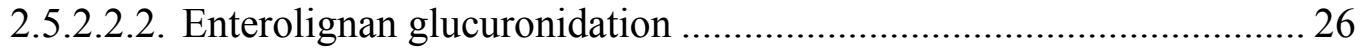

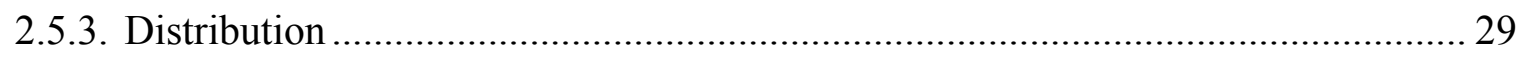

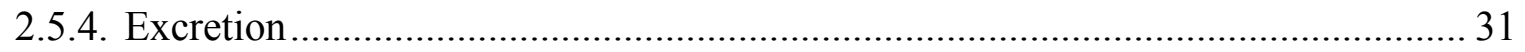

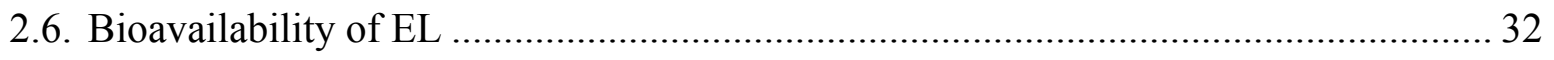

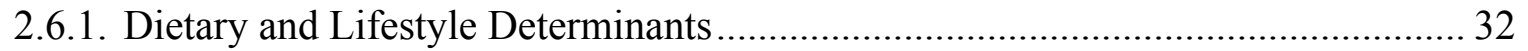

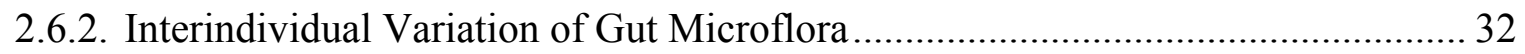

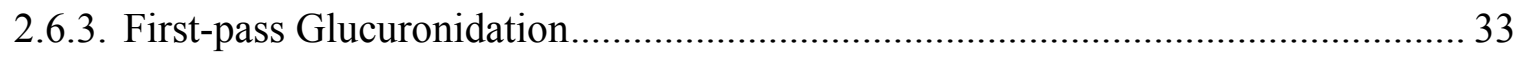

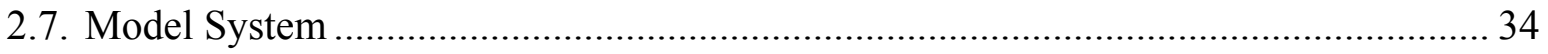

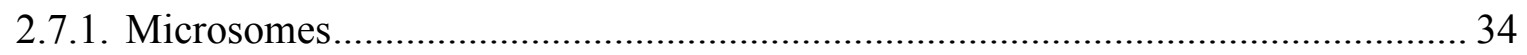

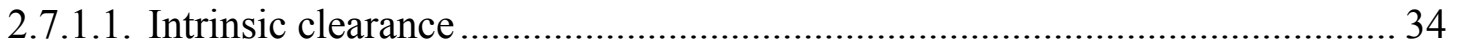

2.7.2. Human versus Rat Models .................................................................................. 35

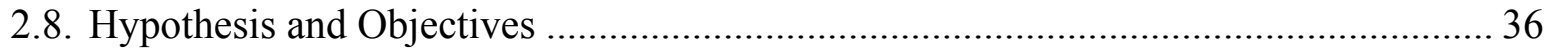

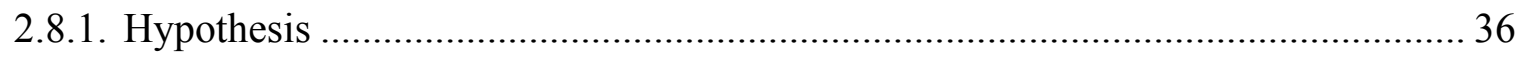

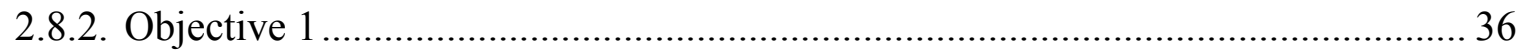

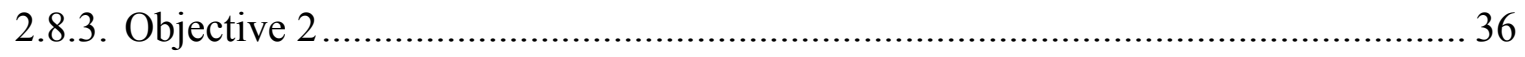

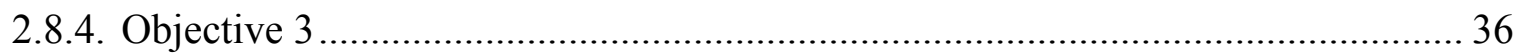

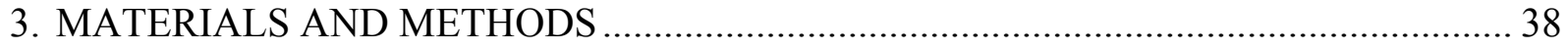

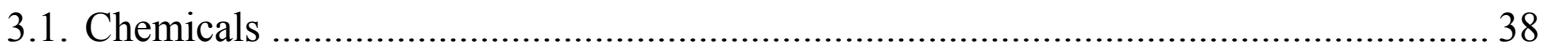

3.2. Experiment one: EL Glucuronide Synthesis and Characterization …........................ 38

3.2.1. Preparation of Rat Liver and Intestinal Microsomes ........................................... 38

3.2.1.1. Preparation of rat liver microsomes........................................................... 38 
3.2.1.2. Preparation of rat intestinal microsomes

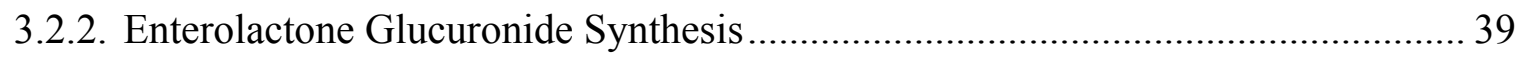

3.2.3. Purification of EL Glucuronide Conjugates ........................................................ 40

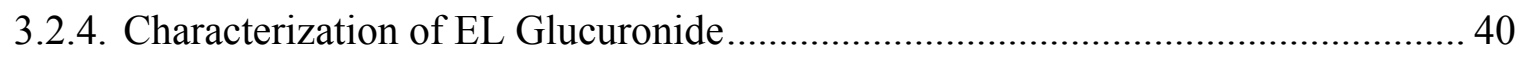

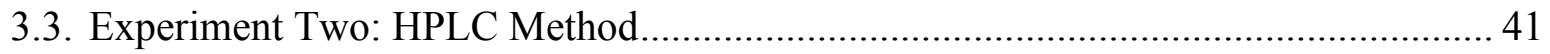

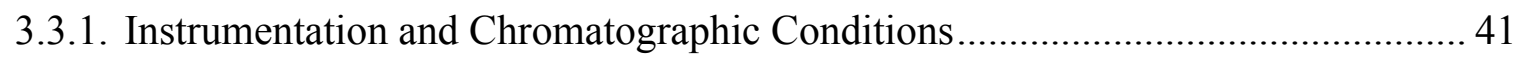

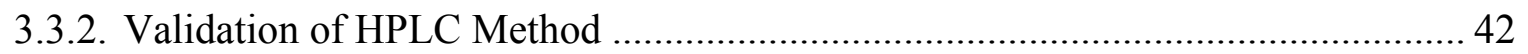

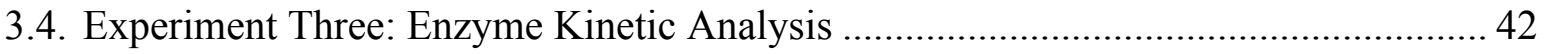

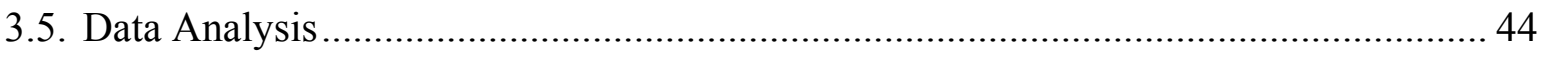

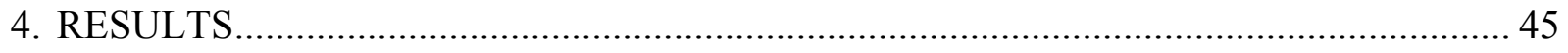

4.1. EL Glucuronide Synthesis with Rat and Human Microsomes ................................... 45

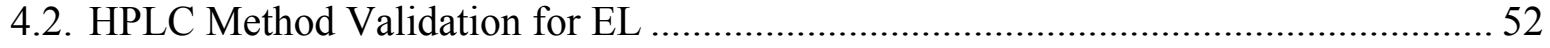

4.3. In Vitro Hepatic and Intestinal Microsomal Intrinsic Clearance of EL ....................... 55

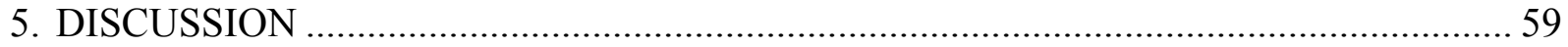

5.1. EL Glucuronide Synthesis and Characterization ..................................................... 59

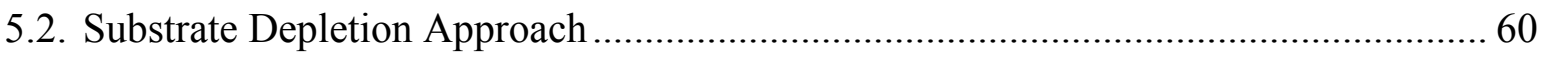

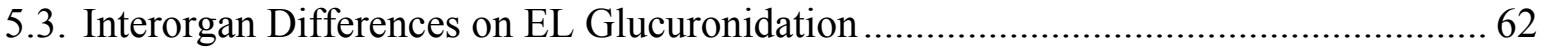

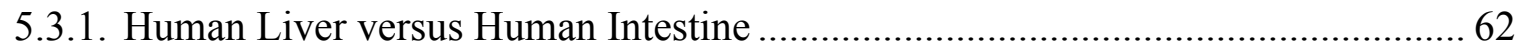

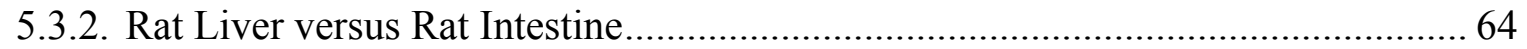

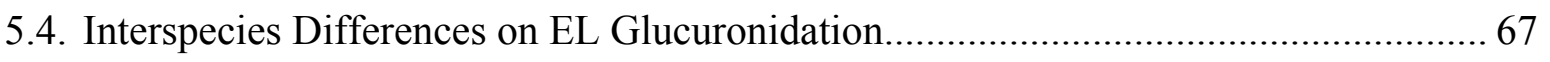

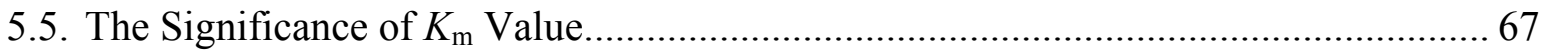

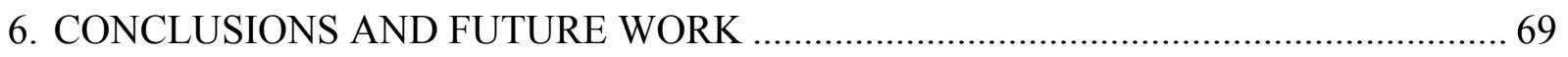

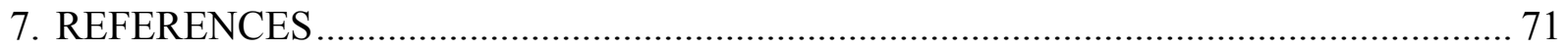

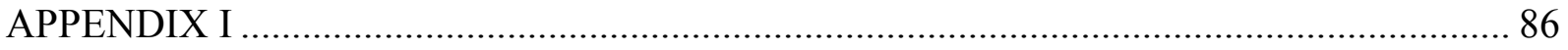




\section{LIST OF FIGURES}

Figure 2.1 The structural makeup of lignans............................................................... 8

Figure 2.2 Pathway of the conversion from plant lignans to mammalian lignans ED and EL9

Figure 2.3 Diagram of the intestinal microflora mediated conversion of lignans in human gastrointestinal tract. 23

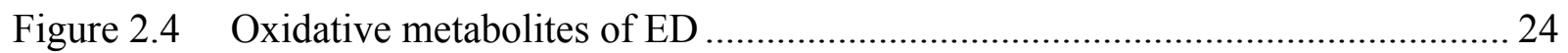

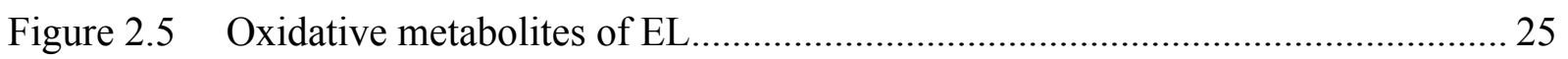

Figure 2.6 Conjugative glucuronidation metabolites of ED ............................................ 28

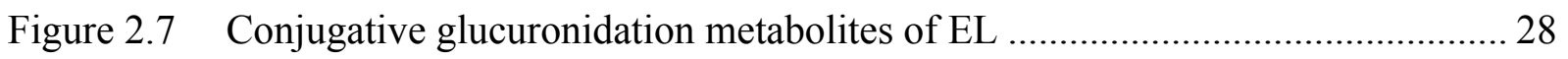

Figure 2.8 The enterohepatic circulation of plant lignans, their colonic and liver metabolites

Figure 4.1 Representative HPLC chromatograms of unpurified EL glucuronide (A) and purified EL glucuronide (B) generated from rat liver microsomes. Conditions: Waters 600 HPLC system; reversed-phase semi-preparative column $(300 \times 10$ mm I.D., $5 \mu \mathrm{m}$ particle size); component A: water with $0.1 \%$ formic acid, component B: acetonitrile with $0.1 \%$ formic acid; detection wavelength at 280 $\mathrm{nm}$. 46

Figure 4.2 LC-MS analysis of proposed EL glucuronide ( $\mathrm{m} / \mathrm{z} 473)$ formed from rat liver microsomes obtained in negative electrospray ionization (ESI) mode 47

Figure 4.3 Multiple reaction monitoring chromatograms of unpurified EL glucuronide (A) and purified EL glucuronide (B) generated from rat liver microsomes (RLM).. 48

Figure 4.4 Multiple reaction monitoring analysis of EL glucuronide incubated with rat duodenum microsomes (RDM) (A), rat jejunum microsomes (RJM) (B), rat colon microsomes (RCM) (C), human liver microsomes (HLM) (D) and human intestinal microsomes (HIM) (E) 51 
Figure 4.5 Representative HPLC chromatograms of blank rat liver microsomes (A) and rat liver microsomes spiked with internal standard umbelliferone $(100 \mu \mathrm{g} / \mathrm{mL}, 6.8$ $\min )$ and EL (4 $\mu \mathrm{g} / \mathrm{mL}, 12.0 \mathrm{~min})(\mathrm{B})$. Conditions: Agilent $1200 \mathrm{HPLC}$ system; Waters Symmetry reversed-phase C18 analytical column $(150 \times 4.6 \mathrm{~mm}$ I.D., 5 $\mu \mathrm{m}$ particle size); component $\mathrm{A}$ : water with $0.1 \%$ formic acid, component $\mathrm{B}$ : acetonitrile with $0.1 \%$ formic acid; excitation wavelength at $277 \mathrm{~nm}$ and emission wavelength at $617 \mathrm{~nm}$. 53

Figure 4.6 Plots of in vitro depletion rate constants $\left(k_{\mathrm{dep}}\right)\left(\mathrm{min}^{-1}\right)$ versus EL concentrations $(\mu \mathrm{M})$ for EL glucuronidation by rat liver microsomes (A), rat jejunum microsomes (B), rat colon microsomes (C), human liver microsomes (D), and human intestinal microsomes $(\mathrm{E})$. Substrate depletion rate constant $\left(k_{\mathrm{dep}}\right)$ for each substrate concentration was equal to the slope of the linear line from a plot of $\ln$ analyte peak area percentage remaining (normalized to the initial peak area obtained at $\mathrm{t}=0$.) versus time. Refer to Appendix I for all $k_{\text {dep }}$ values for various EL concentrations for different organs from different species.

Figure 4.7 The $\mathrm{CL}_{\text {int }}(\mathrm{A})$, and Michaelis-Menten constants, $\mathrm{V}_{\max }(\mathrm{B})$ and $K_{\mathrm{m}}(\mathrm{C})$, for $\mathrm{EL}$ glucuronidation by rat liver microsomes (RLM, n=3), rat jejunum microsomes (RJM, $\mathrm{n}=2$ ), rat colon microsomes ( $\mathrm{RCM}, \mathrm{n}=2)$, commercial human liver microsomes (HLM, $\mathrm{n}=50$ ) and commercial intestinal microsomes (HIM, $\mathrm{n}=13$ )...

Figure A.1 A plot of natural log percent remaining versus time. 86

Figure A.2 A plot of natural log percent remaining versus time. 87 


\section{LIST OF TABLES}

Table 2.1 Study of dietary lignans and enterolignans on breast cancer risk ......................... 12

Table 4.1 Intraday assay precision and accuracy for EL in rat liver microsomes $(\mathrm{N}=6) \ldots \ldots . .54$

Table 4.2 Interday assay precision and accuracy for EL in rat liver microsomes $(\mathrm{N}=6) \ldots \ldots . .54$

Table 4.3 $\mathrm{CL}_{\text {int }}$ and Michaelis-Menten constants of EL glucuronidation by rat and human

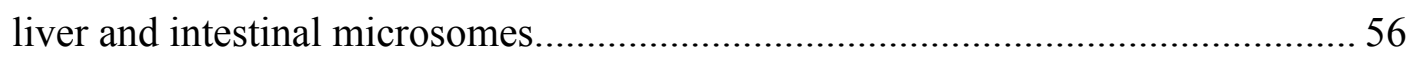

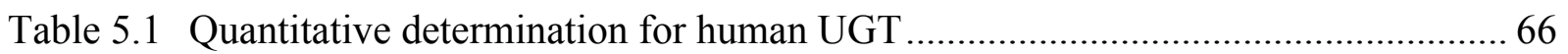

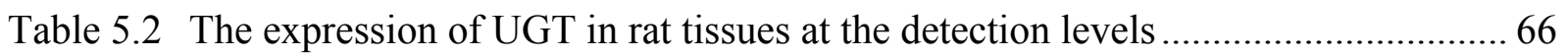

Table A.1 The natural log percent remaining for EL at multiple time points when a low EL concentration was incubated with rat liver microsomes .................................... 86

Table A.2 The natural log percent remaining for EL at multiple time points when a high EL concentration was incubated with rat liver microsomes 87

Table A.3 Various EL concentrations $(10-500 \mu \mathrm{M})$ versus percent remaining for rat liver microsomal kinetic study 88

Table A.4 Various EL concentrations $(1.5-100 \mu \mathrm{M})$ versus percent remaining for rat jejunum microsomal kinetic study 88

Table A.5 Various EL concentrations $(1.5-100 \mu \mathrm{M})$ versus percent remaining for rat colon microsomal kinetic study 88

Table A.6 Various EL concentrations $(10-500 \mu \mathrm{M})$ versus percent remaining for human liver microsomal kinetic study 89

Table A.7 Various EL concentrations $(10-500 \mu \mathrm{M})$ versus percent remaining for human intestinal microsomal kinetic study 89 


\section{LIST OF ABBREVIATIONS}

\begin{tabular}{|c|c|}
\hline $17 \beta$-HSD & 17ß-hydroxysteroid dehydrogenase \\
\hline 4-MU & 4-methylumbelliferone \\
\hline ALA & $\alpha$-linolenic acid \\
\hline AUC & Area under curve \\
\hline BMI & Body mass index \\
\hline BSA & Bovine serum albumin \\
\hline $\mathrm{CaCl}_{2}$ & Calcium chloride \\
\hline Caco-2 & Colon adenocarcinoma cell line \\
\hline CAD & Collision activated dissociation \\
\hline $\mathrm{CE}$ & Collision energy \\
\hline $\mathrm{CL}_{\text {int }}$ & Intrinsic clearance \\
\hline $\mathrm{Cl}_{\mathrm{s}}$ & Systemic clearance \\
\hline $\mathrm{C}_{\max }$ & Maximum drug concentration in the blood \\
\hline CRU & Curtain gas \\
\hline $\mathrm{CV}$ & Coefficient of variation \\
\hline CVD & Cardiovascular disease \\
\hline CXP & Collision cell exit potential \\
\hline $\mathrm{dd}_{2} \mathrm{O}$ & Double distilled water \\
\hline DIN-HM & Homeopathic Medicine Number \\
\hline DHT & ${ }^{3} \mathrm{H}$-dihydrotestosterone \\
\hline DMBA & 9,10-dimethyl-1, 2-benzanthracene \\
\hline DP & Declustering potential \\
\hline E2 & Estradiol \\
\hline ED & Enterodiol \\
\hline EDTA & Ethylenediamine-tetracetic acid \\
\hline EL & Enterolactone \\
\hline EL-Glu & Enterolactone glucuronide \\
\hline
\end{tabular}




\begin{tabular}{|c|c|}
\hline EGCG & Epigallocatechin gallate \\
\hline EN & Exemption Number \\
\hline EP & Entrance potential \\
\hline ER & Estrogen receptor \\
\hline ESI & Electrospray ionization \\
\hline GIT & Gastrointestinal tract \\
\hline GMP & Good Manufacturing Practice \\
\hline GS & Ion source gas \\
\hline $\mathrm{H}_{2} \mathrm{O}_{2}$ & Hydrogen peroxide \\
\hline $\mathrm{HCl}$ & Hydrogen chloride \\
\hline HIM & Human intestinal microsomes \\
\hline HLM & Human liver microsomes \\
\hline HMGA & 3-hydroxy-3-methyl glutaric acid \\
\hline HPLC & High pressure liquid chromatography \\
\hline HQC & High quality control \\
\hline $\mathrm{IC}_{50}$ & Half maximal inhibitory concentration \\
\hline ISV & Ion spray voltage \\
\hline IUPAC & International Union of Pure and Applied Chemistry \\
\hline $\mathrm{KCl}$ & Potassium chloride \\
\hline $\mathrm{k}$ & Elimination rate constant \\
\hline$k_{\mathrm{dep}}$ & Substrate depletion rate constant \\
\hline$k_{\mathrm{dep}([\mathrm{S}] \rightarrow 0)}$ & Theoretical maximum consumption rate constant \\
\hline $\mathrm{KH}_{2} \mathrm{PO}_{4}$ & Monopotassium phosphate \\
\hline $\mathrm{K}_{\mathrm{i}}$ & Inhibition kinetic parameter \\
\hline$K_{\mathrm{m}}$ & Michaelis-Menten constant \\
\hline LARI & Lariciresinol \\
\hline LC-MS & Liquid chromatography-mass spectrometry \\
\hline LLOQ & Lowest limit of quantification \\
\hline LOD & Limit of detection \\
\hline
\end{tabular}




\begin{tabular}{|c|c|}
\hline LQC & Low quality control \\
\hline $\mathrm{m} / \mathrm{z}$ & Mass to charge ratio \\
\hline MAT & Matairesinol \\
\hline $\mathrm{MgCl}_{2}$ & Magnesium chloride \\
\hline MNU & N-methyl-N-nitrosourea \\
\hline MQC & Middle quality control \\
\hline MRM & Multiple reaction monitoring \\
\hline $\mathrm{Na}_{2} \mathrm{HPO}_{4}$ & Disodium phosphate \\
\hline $\mathrm{NaCl}$ & Sodium chloride \\
\hline NDGA & Nordihydroguaiaretic \\
\hline NHP & Natural Health Product \\
\hline NHPD & Natural Health Products Directorate \\
\hline NHPR & Natural Health Products Regulation \\
\hline NPN & Natural Product Number \\
\hline $\mathrm{O}_{2}^{-}$ & Superoxide \\
\hline$\cdot \mathrm{OH}$ & Hydroxyl radical \\
\hline PINO & Pinoresinol \\
\hline PMSF & Phenylmethylsulfonylflouride \\
\hline QC & Quality control \\
\hline $\mathrm{RCM}$ & Rat colon microsomes \\
\hline $\mathrm{RDM}$ & Rat duodenum microsomes \\
\hline RJM & Rat jejunum microsomes \\
\hline RLM & Rat liver microsomes \\
\hline ROS & Reactive oxygen species \\
\hline RSD & Relative standard deviation \\
\hline$[S]$ & Substrate concentration \\
\hline SBP & Sex steroid binding protein \\
\hline SDG & Secoisolariciresinol diglucoside \\
\hline SECO & Secoisolariciresinol \\
\hline
\end{tabular}


SHBG

$\mathrm{t}_{1 / 2}$

TEM

UDPGA

UGT

UV

$\mathrm{V}_{\mathrm{d}}$

$\mathrm{V}_{\max }$
Sex hormone binding globulin

Half life

Temperature

5'-diphosphoglucuronic acid

Uridine diphosphoglucuronosyl transferase

Ultraviolet

Volume of distribution

Maximum velocity 


\section{INTRODUCTION}

Flaxseed is the richest source of dietary intake of the plant lignan secoisolariciresinol diglucoside (SDG). A growing body of evidence suggests that SDG and its metabolites, in particular enterolactone (EL), may exert protective effects against cancer and cardiovascular disease through diverse mechanisms. Epidemiological studies suggest that the lower risk of aforementioned chronic diseases is greatly attributed to the high circulating concentrations of EL. However, the systemic concentration of EL is highly variable. The low and varying bioavailability of EL might be greatly attributed to extensive first-pass metabolism, which is responsible for the low bioavailability of many xenobiotics. In human plasma and urine, the principal conjugates of EL are mainly glucuronides, suggesting a significant first-pass effect by glucuronidation occurring both in hepatocytes and enterocytes. Nevertheless, the exact contributions from liver and intestine are poorly understood.

Hence, a fundamental understanding of EL glucuronidation in the liver and intestine is essential to provide critical information regarding the oral absorption properties of EL, and also possibly to infer the potential for drug-lignan interactions. Therefore, in my project, in vitro enzyme kinetic studies using rat and human liver and intestinal microsomes with determination of intrinsic clearance $\left(\mathrm{CL}_{\mathrm{int}}\right)$, which directly measures the efficacy of enzymes to metabolize substrates, allow the comparison of differences of EL glucuronidation between the two first-pass metabolism organs and among two species.

The literature review on flaxseed lignans contains several sections. The first two sections give an overview of the regulations as well as benefits and risks of Natural Health Products (NHPs) and the status of flaxseed and lignans as NHPs in Canada. It also provides a general framework of the production of EL. The third section demonstrates the health benefits of EL according to the evidence from both epidemiological and experimental animal studies, although the studies are not completely consistent. The next section discusses the various mechanisms responsible for the biological effects of EL. The fifth section essentially deals with the pharmacokinetics (absorption, metabolism, distribution and excretion) of plant and mammalian lignans with an emphasis on the first-pass glucuronidation of EL. The last two sections discuss the low systemic levels of EL and the factors possibly accounting for the low 
bioavailability. The model system is also discussed to explain the significance of including both human and rat models into the study. 


\section{LITERATURE REVIEW}

\subsection{Natural Health Products}

\subsubsection{Introduction of Natural Health Products}

In Canada, Natural Health Products (NHPs) are regulated under the Natural Health Products Regulations (NHPR), which is different from the regulations for drugs and food. The Natural Health Products Directorate (NHPD) in Canada defines NHPs as naturally occurring substances which include vitamins and minerals, herbal remedies, homeopathic medicines, traditional medicines like traditional Chinese and East Indian medicines, probiotics and other products like amino acids and essential fatty acids ${ }^{1}$. NHPs are widely used around the world, with increasing use in Western countries, to ensure health and wellness and/or for their putative medical benefits. A recent survey conducted in 2010 showed that $73 \%$ of Canadians were using NHPs with greater prevalence in older than younger populations ${ }^{2}$.

\subsubsection{Regulation of Natural Health Products}

Each NHP must receive market authorization by obtaining a product license based on evidence that the product is safe under the recommended conditions of use without a prescription, effective for the proposed claims, and of high quality ${ }^{3}$. NHPD requires evidence, which might vary depending on the product and type of health claim, to support the safety and efficacy of the NHP according to its recommended conditions of use prior to approval for sale in Canada. That evidence must come from human use; animal or in vitro experimental evidence may be considered as additional supporting information but cannot be the basis for approval $^{4}$. For health support claims implying treatment, cure, prevention, and risk reduction claims related to a major or serious condition, for example a health claim of a chemopreventive NHP for cancer prevention, the NHPD requires at least two Phase III trials (randomized, controlled, well-designed) and two prospective observational studies ${ }^{5}$. Other information packages that reflect the totality of evidence, including systematic literature review, various epidemiological studies, background information and safety data with respect to in vitro experiments and animal studies are demanded in support of a requested claim ${ }^{5}$. 
Once a product has been assessed the ingredients and claims to ensure safety, efficacy and a product of high quality, an eight-digit Natural Product Number (NPN) or Homeopathic Medicine Number (DIN-HM) will be granted, indicating that the product is reviewed and approved by Health Canada ${ }^{6}$. Consumers can identify and/or search for licensed products for sale in Canada by looking for the NPN or DIN-HM on the label and/or Health Canada's Licensed Natural Health Products Database. In addition to the product license, each NHP sold in Canada requires a site license in order to manufacture, package, label and import for sale, which should comply with the guidance of Good Manufacturing Practices (GMPs) specifically for NHPs ${ }^{3}$. Since Health Canada has not yet evaluated all NHPs currently on the market, products with exemption numbers $(\mathrm{EN})$ can also be sold in Canada. Except for the pre-market assessment, Health Canada also requires product license holders to report any serious adverse reactions and any serious unexpected adverse reaction to their products ${ }^{3}$.

\subsubsection{Benefits and Risks of Natural Health Products}

NHPs are prevalently employed with drug therapies by consumers to promote health and treat diseases such as cold, inflammation, pain, heart diseases, liver cirrhosis, diabetes and central nervous system diseases ${ }^{7}$. The principal reason for the progressive use of NHPs is mainly due to the belief that they are natural and, therefore, must be safe and nontoxic. While NHPs are generally safe and have few side effects, they are not free of risk. Life threatening adverse reactions associated with NHPs have been discovered and reported with increasing incidence. Adverse effects can arise from an inherent toxicity of NHPs, manufacturing problems like contamination, incorrect ingredients or dosage levels, adulteration with pharmacologically active synthetic compounds, and interactions with prescription drugs or other NHPs which may lead to the loss of therapeutic efficacy, toxicity or even mortality ${ }^{8}$.

\subsubsection{Natural Health Product-drug interactions}

An important risk of NHP usage is the potential for NHP-drug interactions. Numerous studies have documented the interactions of NHPs with Phase I enzymes, particularly the Cytochrome P450 (P450) enzymes. For instance, St. John's wort, used worldwide in the 
treatment of mild to moderate depression, induces CYP3A4, CYP2E1 as well as CYP2C19 and causes interactions with cyclosporine or antiretrovirals that are substrates of aforementioned P450 enzymes ${ }^{9}$. Kava is another commonly used NHP in Western countries as a result of its anxiolytic and sedative properties ${ }^{10}$. Recently, studies suggest that several kavalactones, the assumed active ingredients of kava extracts, are potent inhibitors of several enzymes of the P450 system (CYP1A2, 2C9, 2C19, 2D6, 3A4 and 4A9/11), which implies kava has a great potential to cause interactions with drugs that are mainly metabolized by the foregoing P450 enzymes ${ }^{11}$.

Apart from the large numbers of P450 enzyme-mediated NHP-drug interactions, emerging evidence has suggested the high potential of Phase II enzymes, principally uridine diphosphoglucuronosyl transferases (UGT), to cause NHP-drug interactions. Valerian and valerian/hops, popular over-the-counter products for the treatment of sleep disturbances or anxiety, have the potential to cause NHP-drug interactions by inhibiting UGT. Significant inhibitory effects on the glucuronidation of acetaminophen, oestradiol, and morphines with both microsomes and expressed UGT in the presence of valerenic acid were observed, and marked reductions were found in UGT1A1 and UGT2B7 activities ${ }^{12}$. Celastrol, a promising anti-tumor agent, exhibited strong inhibitory effects on UGT1A6 and UGT2B7-mediated 4-methylumbelliferone (4-MU) glucuronidation. The low inhibition kinetic parameters $\left(\mathrm{K}_{\mathrm{i}}\right.$ of 0.49 and $0.045 \mu \mathrm{M}$ for UGT1A6 and 2B7, respectively) indicate the possibility of celastrol-drug and/or celastrol-containing herb-drug interactions at the therapeutic concentration of celastrol for anti-tumor utilization ${ }^{13}$. Other significant inhibitors of UGT enzymes include Epigallocatechin gallate (EGCG) for UGT1A4, milk thistle for both UGT1A6 and UGT1A9, saw palmetto (the extracts of its fruits for the treatment of benign prostatic hyperplasia) for UGT1A6, and cranberry for UGT1A9 ${ }^{14}$.

A growing concern exists among both consumers and healthcare practitioners due to the risk of NHP-drug interactions. Hence, the safety assessment of NHPs is of great importance to ensure the safe use of NHPs by consumers. However, the safety assessments of NHPs are not simple due to the compositional diversity of NHPs, their product quality as well as lack of standardization, and limited information is available on the pharmacological data, underlying mechanisms of actions and toxicities ${ }^{15}$. More work is needed on clinical research and toxicity 
studies of NHPs to gain a greater understanding of their pharmacokinetics, efficacy and safety data. Moreover, it is the consumer's responsibility to consult and report the side effects to their healthcare professionals in order to minimize the risks of toxicity or NHP-drug interactions; meanwhile, healthcare professionals should be fully equipped with a thorough knowledge of NHPs to counsel their patients ${ }^{16}$.

\subsubsection{Standardization of Natural Health Products}

Since NHPs are exceptionally complicated and contain multiple active phytochemicals with distinct pharmacological efficacy and toxicological effects, the pharmacological studies and clinical trials involving the identification of the active ingredients, mechanisms of actions, NHP-drug interactions and toxicity are very challenging. A principal problem impeding this progress is the absence of standardization of NHPs (both on materials and analytical methods $)^{17}$. Recognition of the natural variation and proportions of the many active components in the raw material and correct identification of the main components responsible for the desired pharmacological effects are very critical in the standardization process, which ensures consistent quality and adequate levels of the key components for subsequent clinical investigation $^{15,17}$. Validated methods are vital for the evaluation of pharmacological, toxicological and clinical studies of major ingredients ${ }^{17}$. Therefore, the standardization of NHPs is crucial to guarantee the reliability, consistency and reproducibility of the ingredients of NHPs for further clinical research.

\subsection{Flaxseed Lignans}

\subsubsection{Flaxseed}

Flax (Linum usitatissimum) is abundant in fat, protein and dietary fiber. An analysis of brown Canadian flax averaged $41 \%$ fat, $20 \%$ protein, $28 \%$ total dietary fiber, $7.7 \%$ moisture and $3.4 \% \mathrm{ash}^{18}$. The composition of flax can vary with genetics, growing environment, seed processing and method of analysis ${ }^{19}$. 


\subsubsection{The status of flaxseed and lignans as Natural Health Product in Canada}

Flax products such as oil, whole seeds or milled seeds are one of the most popular functional foods used by consumers due to the putative health benefits against degenerative diseases such as cardiovascular disease $(\mathrm{CVD})^{20,21}$ and cancer $^{22,23}$. Various flaxseed oil softgels or capsules and a flax laxative granule have been granted an NPN by Health Canada, and are currently available on the market ${ }^{24}$. Other flax products, for instance, golden flax seed and high lignan flax oil, can be purchased but are not approved by Health Canada.

Flaxseed is a rich source of soluble fiber, $\alpha$-linolenic acid (ALA) and plant lignan secoisolariciresinol diglucoside (SDG), the components of which are proposed to be responsible for the biological effects of flaxseed ${ }^{25}$. However, researchers show a growing interest in lignans because of the significant health benefits associated with them. Though a large number of epidemiological studies and some in vitro models as well as animal studies have shown flaxseed lignans play an important role in the prevention of $\mathrm{CVD}^{26-28}$, diverse cancers $^{29-31}$, oxidative stress and inflammation ${ }^{32,33}$ and diabetes ${ }^{34,35}$, flaxseed lignans or the purified lignans such as SDG have not been given an NPN, for clinical trials suggesting safety and efficacy data are insufficient and pharmacological properties (ADME), toxicities as well as drug interactions are not adequately studied.

\subsubsection{Dietary Lignans}

According to the International Union of Pure and Applied Chemistry (IUPAC), lignans are a large group of natural products derived from cinnamic acid residues, which are characterized by the coupling of two $\mathrm{C}_{6} \mathrm{C}_{3}$ units as propylbenzene based on nomenclature purposes $^{36}$ (Figure 2.1). Dietary plant lignans exist substantially in foods such as oilseeds, cereal grains, legumes, fruits, vegetables and beverages, but ground flaxseed is the richest source of the plant lignan secoisolariciresinol diglucoside (SDG), with pinoresinol (PINO) and matairesinol (MAT) as minor lignan components ${ }^{37-40}$. In Canada, secoisolariciresinol (SECO), MAT, PINO, and lariciresinol (LARI) content in 121 foods were analyzed and data were presented on as is (wet) basis per $100 \mathrm{~g}$ and per serving ${ }^{40}$. Based on the study, the richest sources of lignans were flaxseed $(379 \mathrm{mg} / 100 \mathrm{~g})$ and sesame seed $(8 \mathrm{mg} / 100 \mathrm{~g})$, followed by 
cereals and breads (0.002-7.2 mg/100 g), legumes $(0.002-1 \mathrm{mg} / 100 \mathrm{~g})$, vegetables $(0.001-0.6$ $\mathrm{mg} / 100 \mathrm{~g})$, fruits $(0.002-0.4 \mathrm{mg} / 100 \mathrm{~g})$, and nonalcoholic and alcoholic beverages (0.9-37.3 $\mu \mathrm{g} / 100 \mathrm{~g})^{40}$.<smiles>O=C(O)/C=C/c1ccccc1</smiles>

Cinnamic acid<smiles>CCCc1ccccc1</smiles>

$\mathrm{C}_{6} \mathrm{C}_{3}$ unit, propylbenzene

Figure 2.1 The structural makeup of lignans

\subsubsection{Production of Enterolignans}

Following administration, dietary lignans undergo transformation by the intestinal bacteria before being absorbed into the systemic circulation. As the major plant precursor in flaxseed, the SDG complex and then SDG undergo hydrolysis in the gastrointestinal tract (GIT) to yield the aglycone plant lignan SECO. SECO then undergoes further metabolism to the mammalian lignans, ED and EL, and ED can be oxidized to form $\mathrm{EL}^{29,41}$. As the minor lignan components in flaxseed, MAT, PINO and LARI can also be converted to EL in the GIT through one and several additional steps, respectively ${ }^{42}$ (Figure 2.2). A growing body of evidence suggests that SDG and its metabolites, in particular EL, may exert protective effects against cancers such as prostate, breast and colon cancer ${ }^{22,31,43,44}, \mathrm{CVD}^{26,28}$, inflammation ${ }^{45}$, and oxidative damage through diverse mechanisms including phytoestrogenic ${ }^{46}$ and antioxidant effects ${ }^{47}$ from both in vitro and in vivo studies. Epidemiological studies also suggest that the lower risk of the aforementioned chronic diseases is greatly attributed to high circulating concentrations of $\mathrm{EL}^{48-51}$. Hence, as the promising bioactive form of lignan, the health benefits, mechanisms of the biological effects as well as the pharmacokinetic properties of EL will be discussed below. 


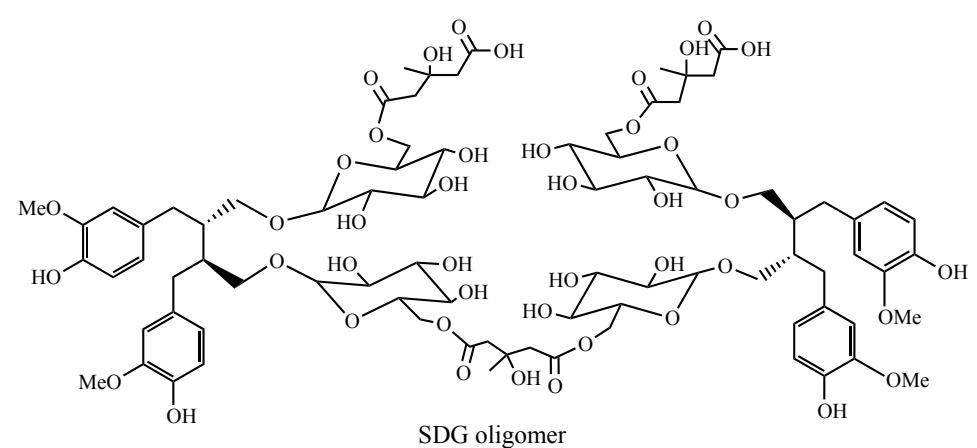

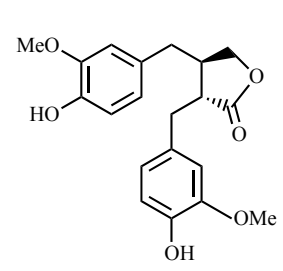

MAT

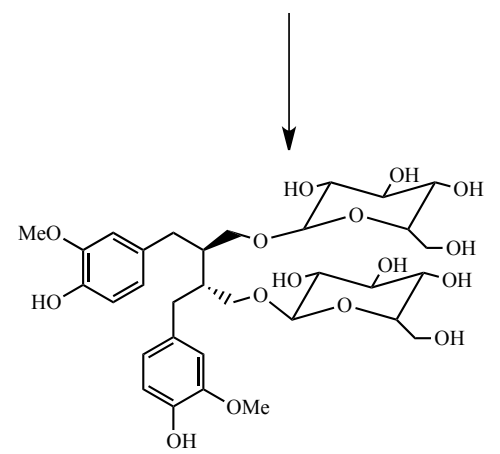

SDG

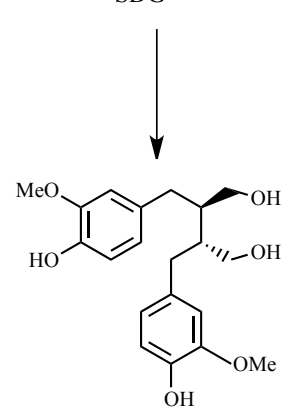

SECO<smiles>CCC(C)Cc1cccc(O)c1</smiles>

ED

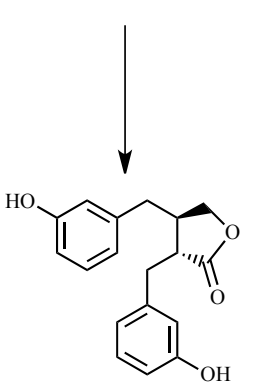

EL

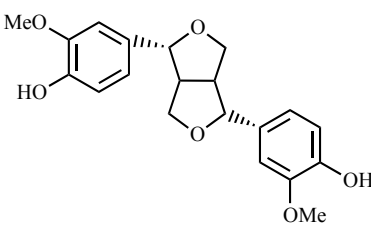

PINO

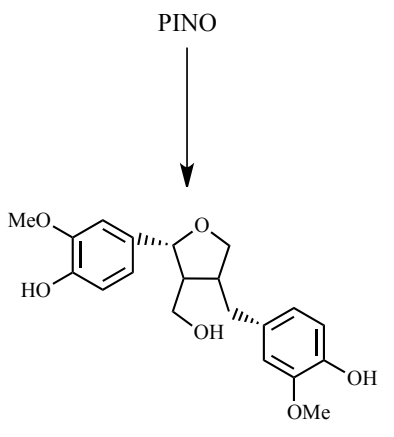

LARI

Figure 2.2 Pathway of the conversion from plant lignans to mammalian lignans ED and EL 


\subsection{EL-Health Benefits}

Compelling data from epidemiological, experimental and clinical studies, suggests that lignans, particularly EL, are promising in the prevention of chronic diseases, although the results concerning the health benefits of lignans, especially from epidemiological studies, are not completely accordant due to the distinct diet habits in populations, varying lignan product quality and intake levels used for the research, lack of flaxseed product standardization and valid analytical methods and different experimental designs ${ }^{52}$.

\subsubsection{Epidemiological Studies}

Epidemiological studies have suggested the promising anti-cancer effects by large lignan consumption and/or high circulating EL levels in the plasma, although some controversial studies exist (Table 2.1). Many studies suggest high intake of lignan-rich foods, which contain plant precursors such as SECO, MAT, LARI, is significantly associated with reduction of breast cancer $^{53-58}$, while some studies are in opposition to this viewpoint ${ }^{59-61}$. By measuring serum or urinary excretion of enterolignans, especially EL, numerous studies demonstrate high plasma concentrations $(54 \mathrm{nM}) /$ urinary excretion levels of EL are inversely correlated with breast $^{31,48,49}, \operatorname{colon}^{50}$ and prostate cancer ${ }^{51}$ risk. However, Kilkkinen and coworkers conducted a nested case-control study in Finland, and their findings were against the hypothesis that high serum EL concentrations were associated with reduced breast cancer risk ${ }^{62}$. Tonkelaar and coworkers also found that higher urinary EL excretion was weakly and nonsignificantly associated with an increased breast cancer risk, but this study included a small number of cases $(88)^{63}$. In another population-based case-referent study in Sweden, Hultén showed that very low plasma level of EL ( $2.9 \mathrm{nM})$ was associated with an increased breast cancer risk in all three cohorts, while very high EL plasma levels $(58.2 \mathrm{nM})$ were also associated with an increased breast cancer risk in two cohorts ${ }^{64}$. The conflicting epidemiological results are in part due to inadequate databases used in intake estimation, dietary habits, lifestyles and lignan-converting bacteria in different populations ${ }^{40}$. It is notable that a clinical trial by Thompson and coworkers showed the potential to reduce tumor growth in patients with breast cancer supplemented with flaxseed, the richest dietary source of lignans ${ }^{23}$. 


\subsubsection{Experimental Animal Studies}

Compared with epidemiological studies, the experimental results from animal models regarding health benefits of lignans are more consistent. In an early study, dietary supplementation with high intake of flaxseed $(5 \%)$ or SDG reduced N-methyl-N-nitrosourea $(\mathrm{MNU})$-induced mammary tumor size and number in rats ${ }^{65}$. Exposure to a diet with $10 \%$ flaxseed or SDG (equivalent to the amount in 10\% flaxseed) during suckling suppressed 9,10-dimethyl-1, 2-benzanthracene (DMBA)-induced rat mammary tumorigenesis and enhanced mammary gland differentiation, suggesting that exposure to lignans at early stage of mammary gland development reduces susceptibility to mammary carcinogenesis later in life $\mathrm{f3}^{43,66}$. Supplementation with 2.5 or $5 \%$ flaxseed or 2.5 or $5 \%$ defatted flaxseed increased cecal $\beta$-glucuronidase activity and significantly reduced the number of aberrant crypts per focus in the distal colon of rats, which implies the colon cancer protective effect of flaxseed may be associated with increased $\beta$-glucuronidase activity ${ }^{29}$. In addition, a series of studies by Prasad and coworkers have demonstrated both flax lignan complex and SDG have beneficial effects in protecting against atherosclerosis ${ }^{26,27,67}$. 
Table 2.1 Study of dietary lignans and enterolignans on breast cancer risk (Adapted from Buck ${ }^{68}$ )

\begin{tabular}{|c|c|c|c|}
\hline 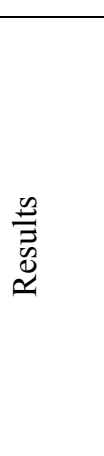 & 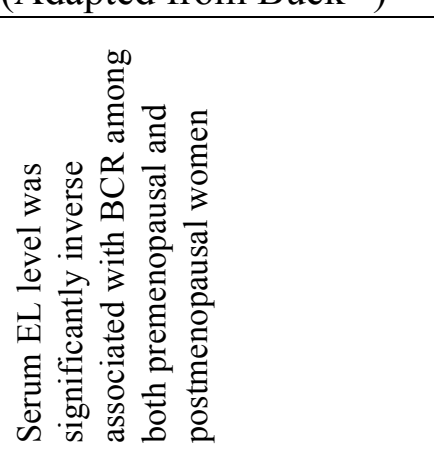 & 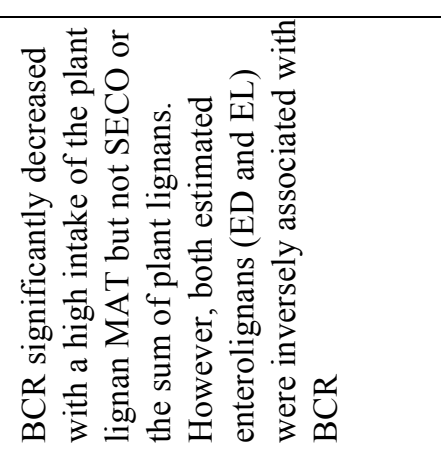 & 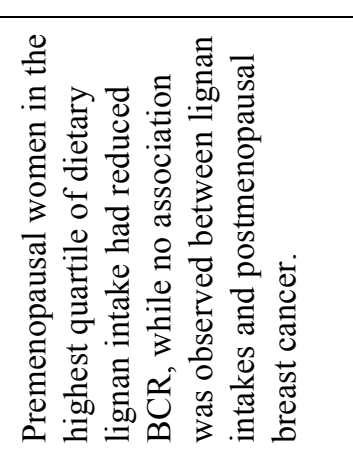 \\
\hline 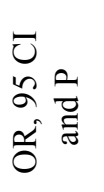 & 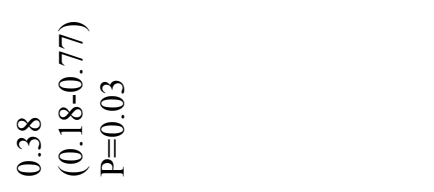 & 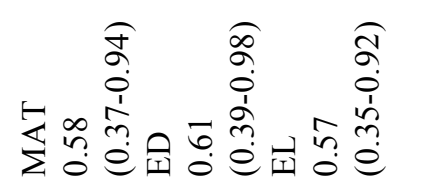 & 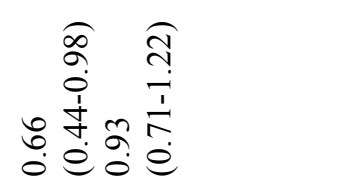 \\
\hline 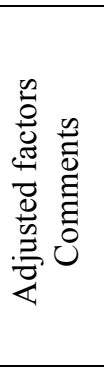 & 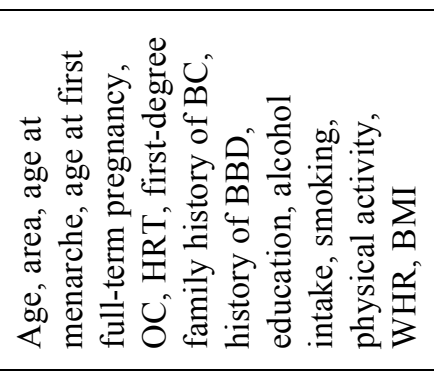 & 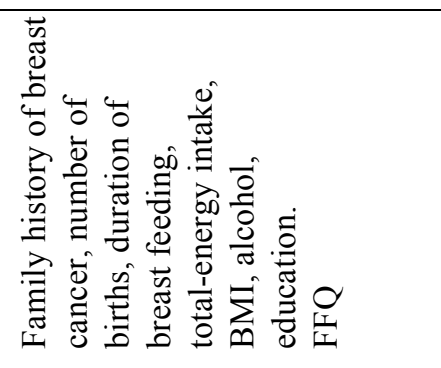 & 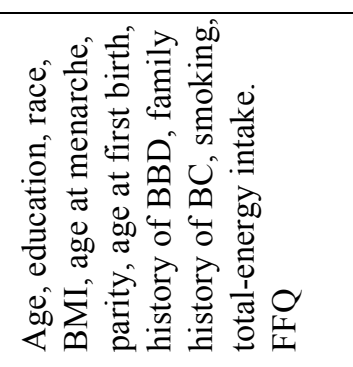 \\
\hline 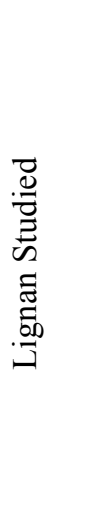 & 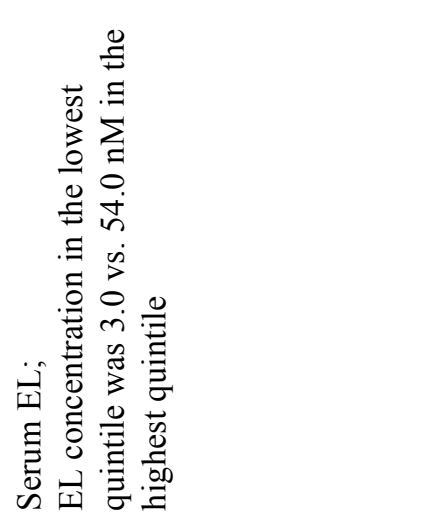 & 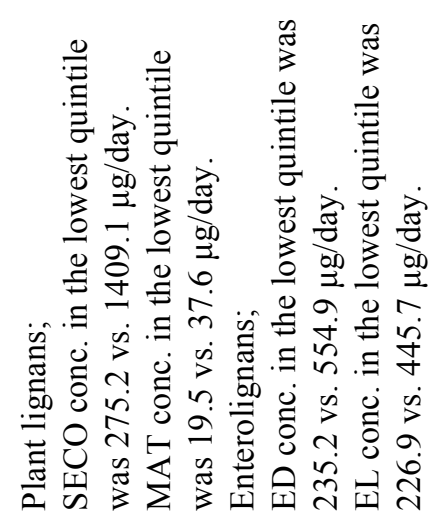 & 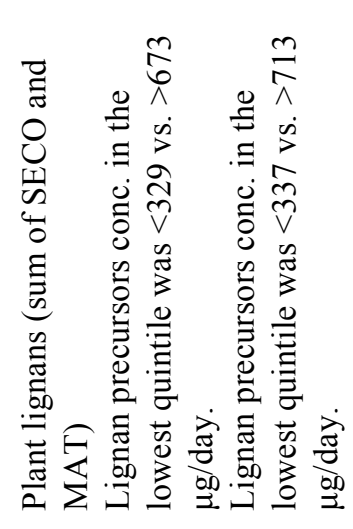 \\
\hline 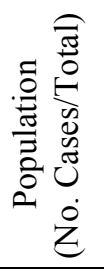 & 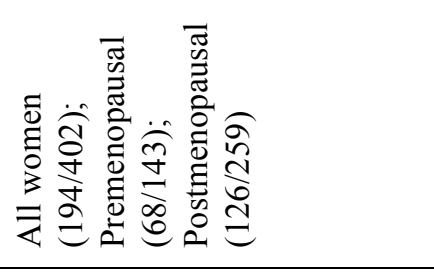 & 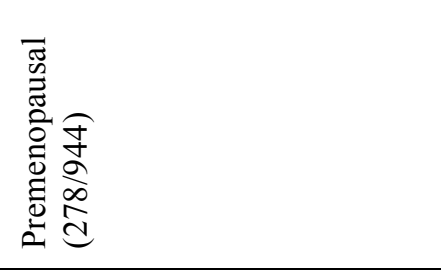 & 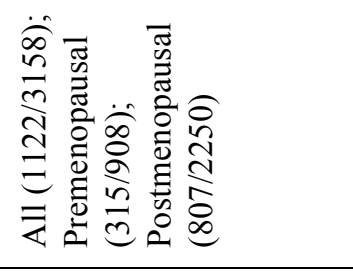 \\
\hline 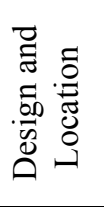 & 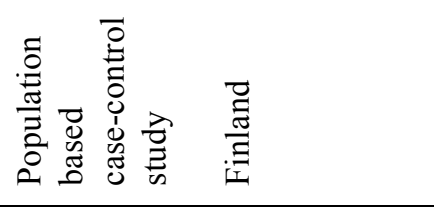 & 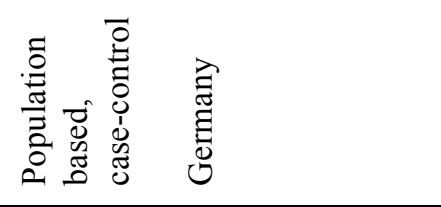 & 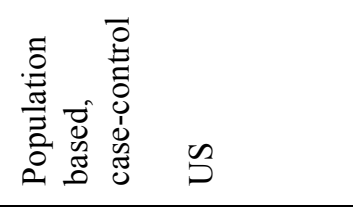 \\
\hline $\begin{array}{l}\stackrel{\vec{t}}{\vec{D}} \\
\stackrel{\vec{n}}{2}\end{array}$ & 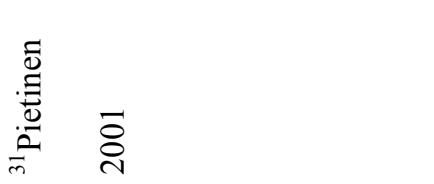 & 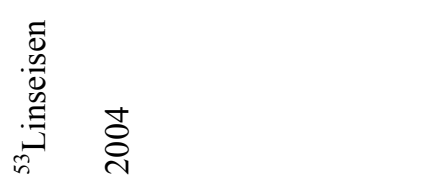 & 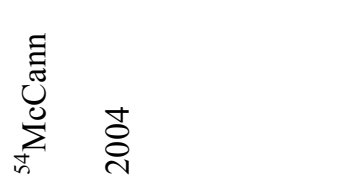 \\
\hline
\end{tabular}


Table 2.1 (Continued)

\begin{tabular}{|c|c|c|c|}
\hline 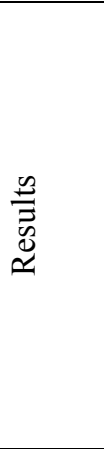 & 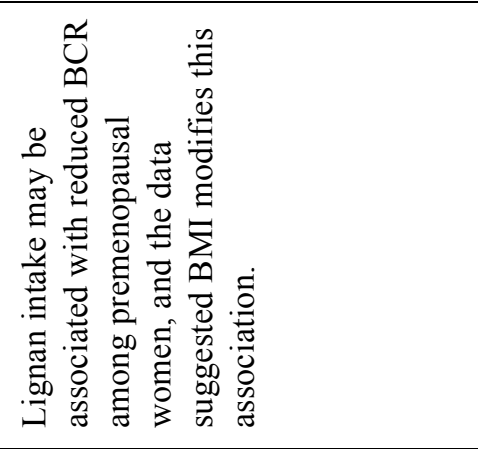 & 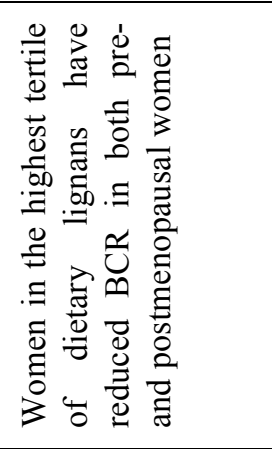 & 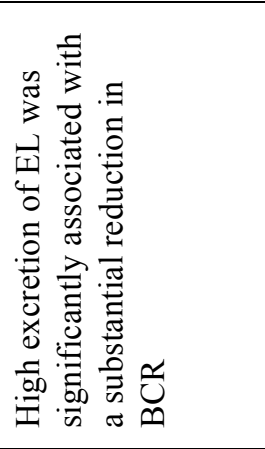 \\
\hline 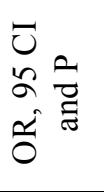 & 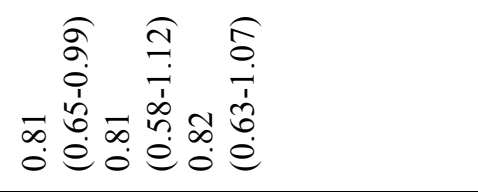 & 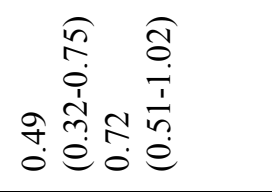 & 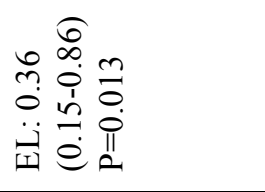 \\
\hline 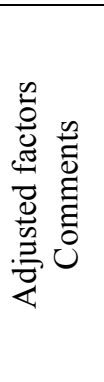 & 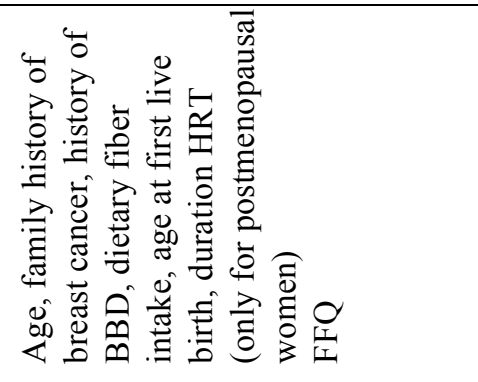 & 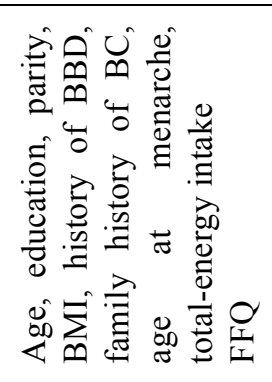 & 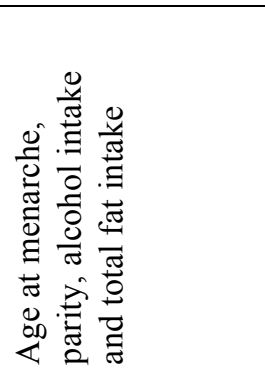 \\
\hline 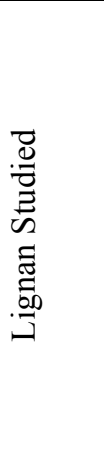 & 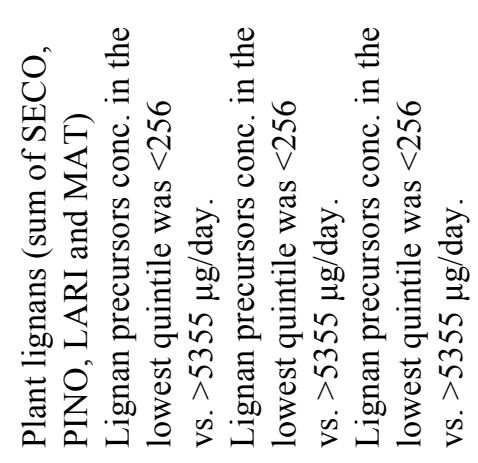 & 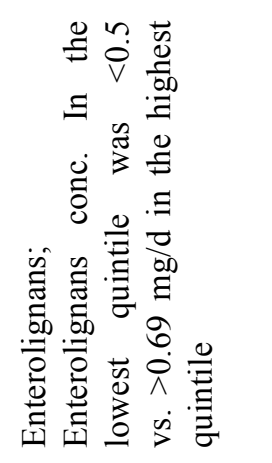 & 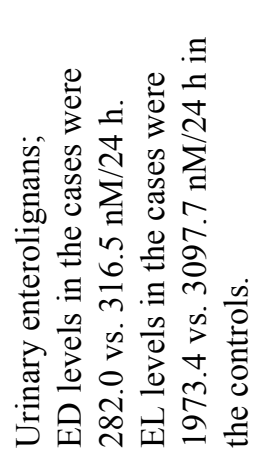 \\
\hline 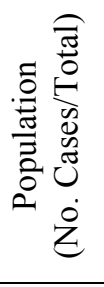 & 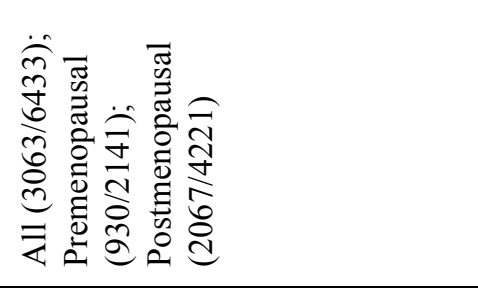 & 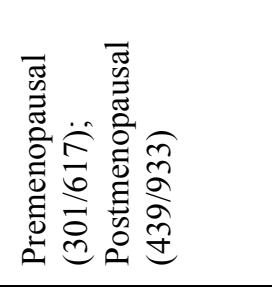 & 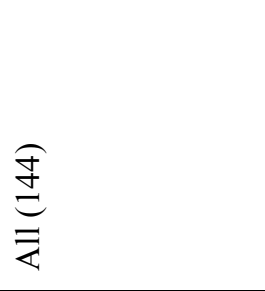 \\
\hline 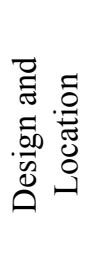 & 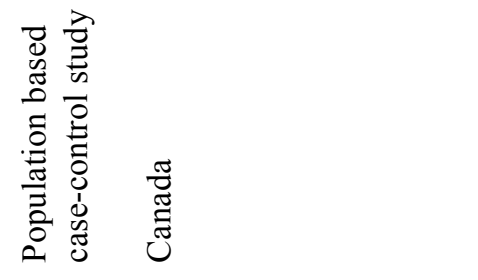 & 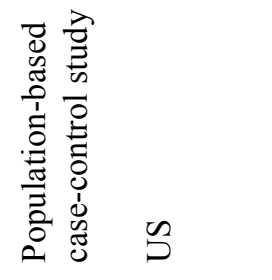 & 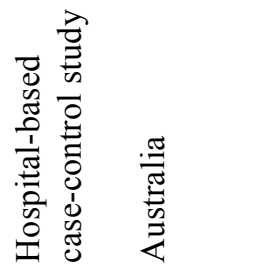 \\
\hline $\begin{array}{l}\stackrel{\vec{D}}{\vec{D}} \\
\stackrel{\vec{n}}{n}\end{array}$ & 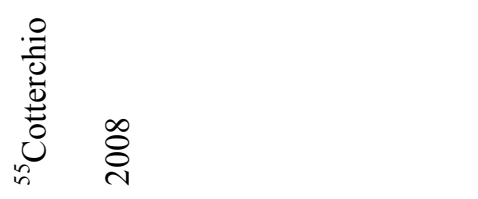 & 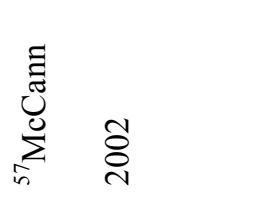 & 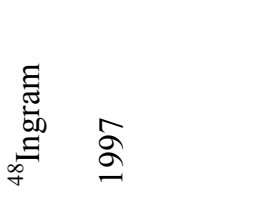 \\
\hline
\end{tabular}


Table 2.1 (Continued)

\begin{tabular}{|c|c|c|c|}
\hline 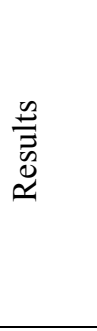 & 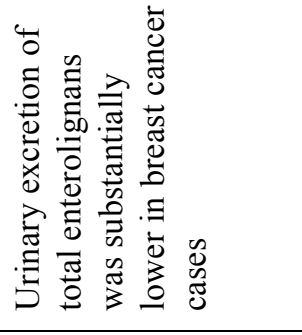 & 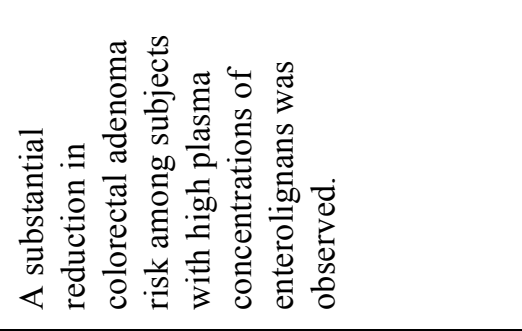 & 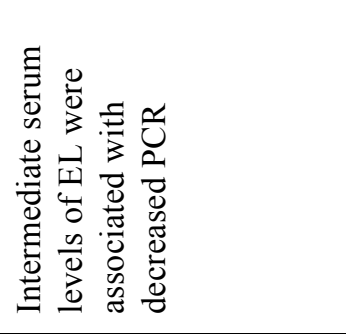 \\
\hline 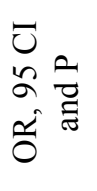 & 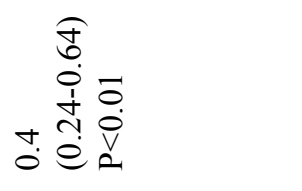 & 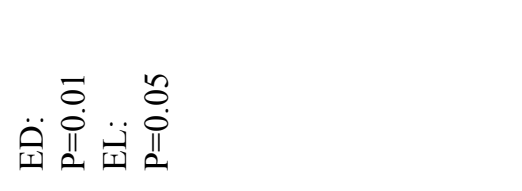 & 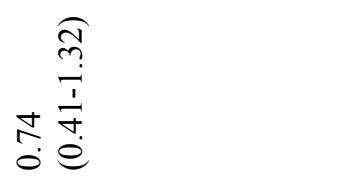 \\
\hline 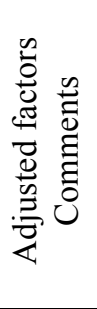 & 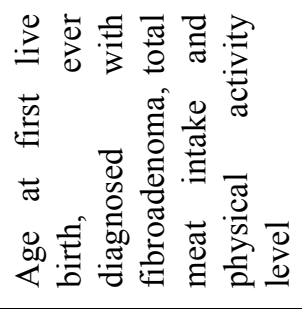 & 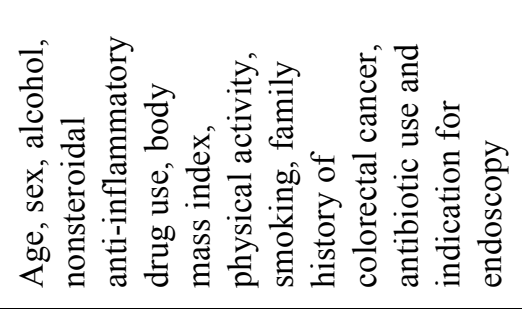 & 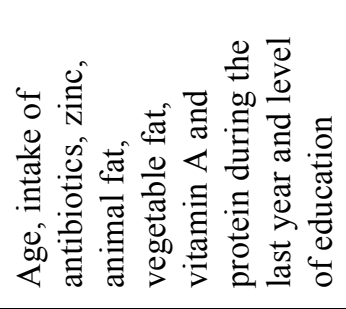 \\
\hline 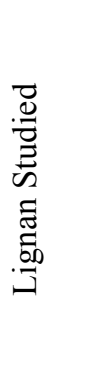 & 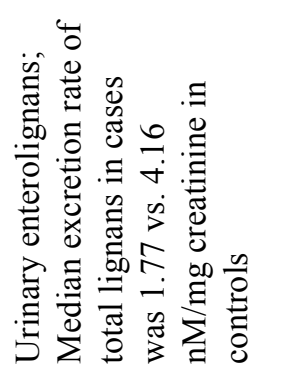 & 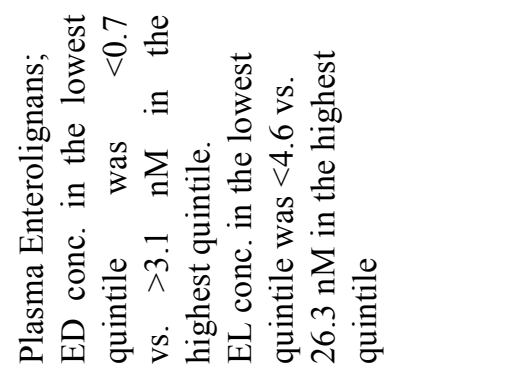 & 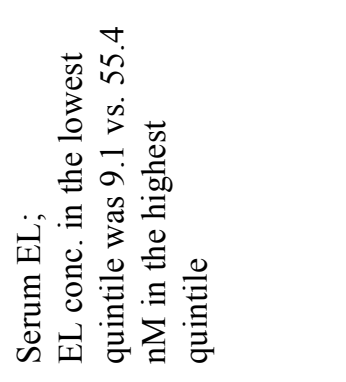 \\
\hline 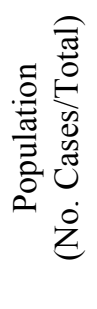 & 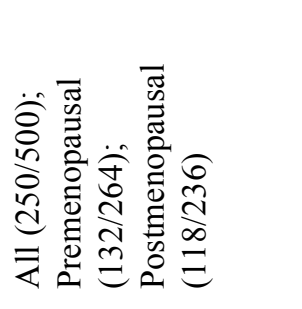 & 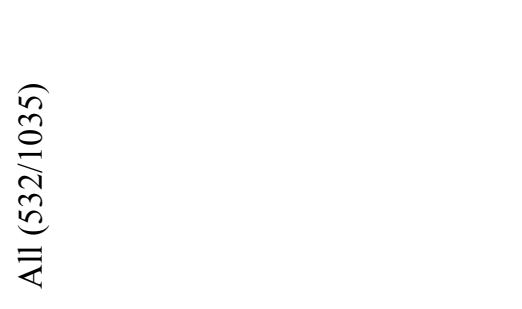 & 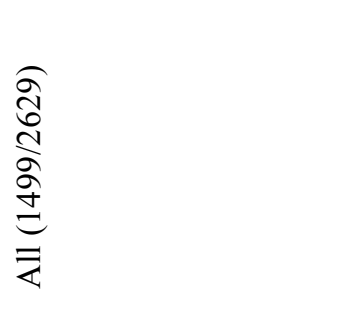 \\
\hline 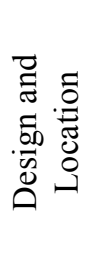 & 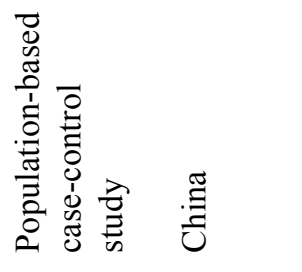 & 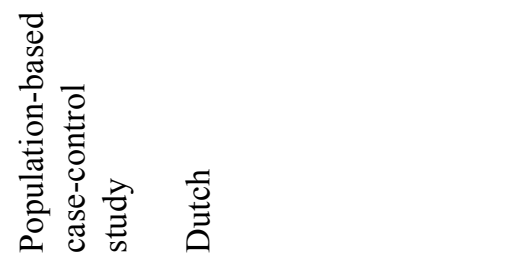 & 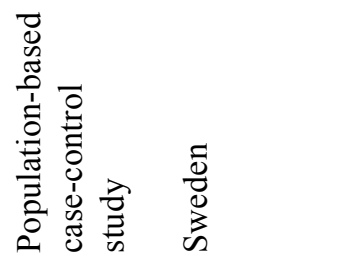 \\
\hline $\begin{array}{l}\vec{z} \\
\stackrel{D}{E}\end{array}$ & ڤึ & 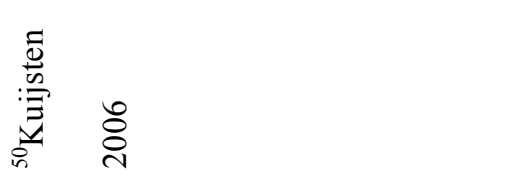 & 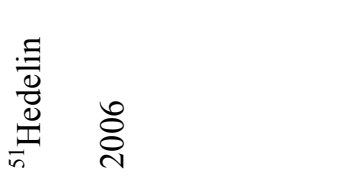 \\
\hline
\end{tabular}


Table 2.1 (Continued)

\begin{tabular}{|c|c|c|c|}
\hline 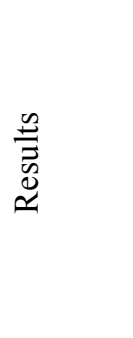 & 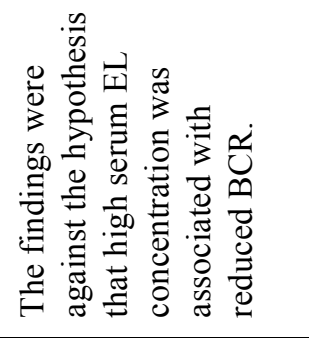 & 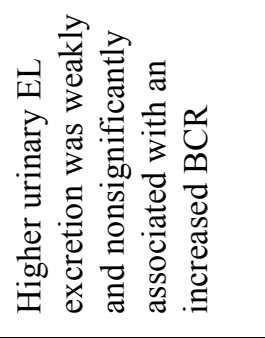 & 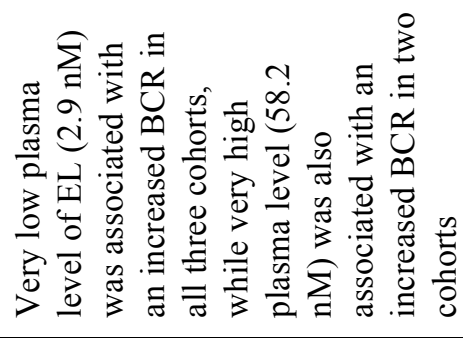 \\
\hline 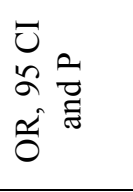 & 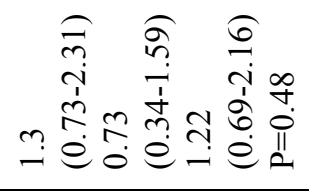 & 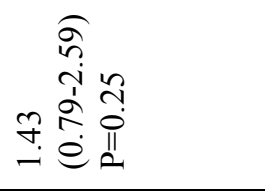 & 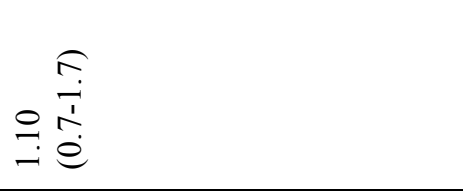 \\
\hline 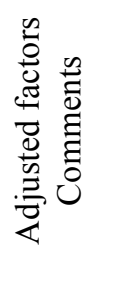 & 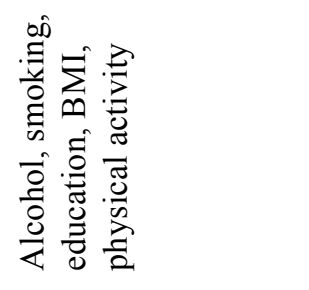 & 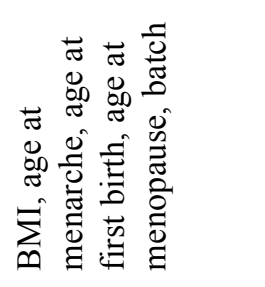 & 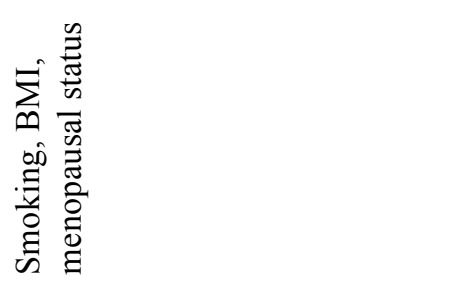 \\
\hline 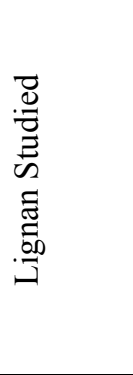 & 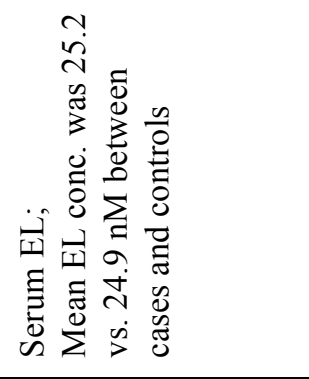 & 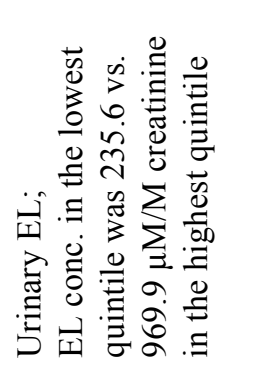 & 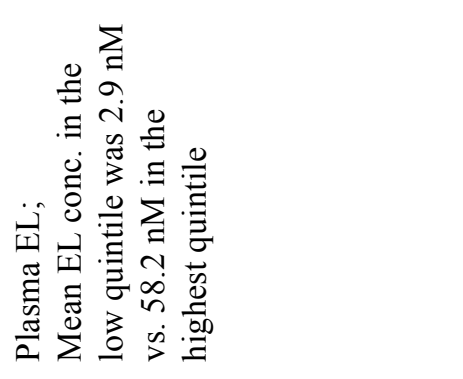 \\
\hline 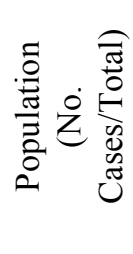 & 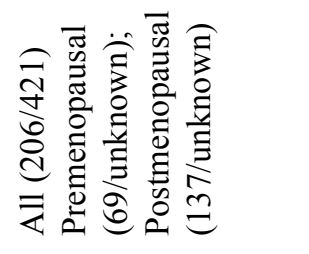 & 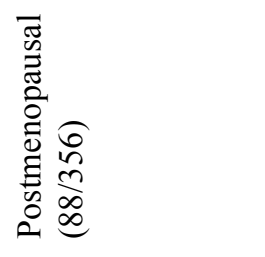 & 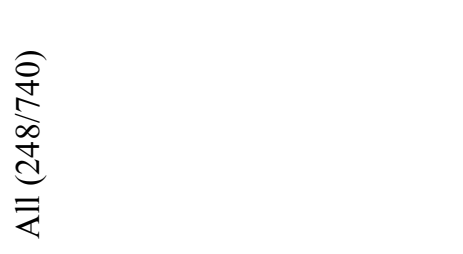 \\
\hline 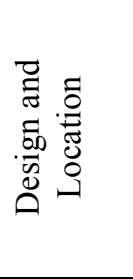 & 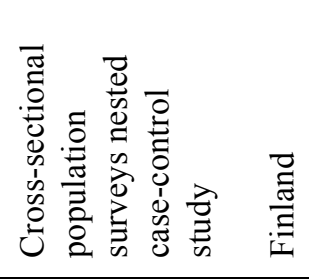 & 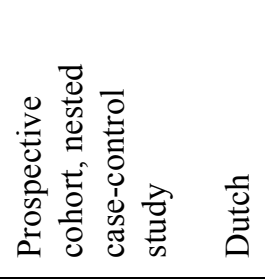 & 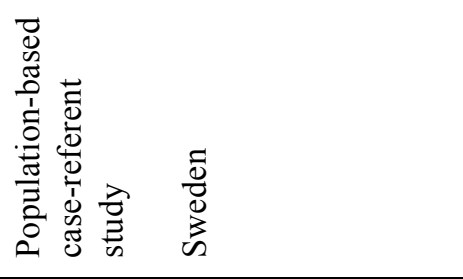 \\
\hline $\begin{array}{l}\overrightarrow{\vec{V}} \\
\stackrel{\vec{E}}{\hat{n}}\end{array}$ & 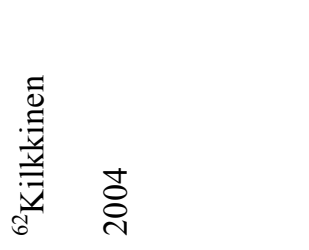 & 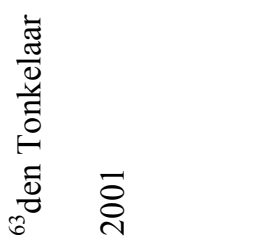 & 苞 於 \\
\hline
\end{tabular}

OR, odds ratio; CI, confidence interval; OC, use of oral contraceptives; HRT, use of hormone replacement therapy; $\mathrm{BC}$, breast cancer; BBD, benign breast disease; WHR, waist-to-hip ratio; BMI, body mass index; FFQ, food-frequency questionnaire; BCR, breast cancer risk; PCR, prostate cancer risk. 


\subsection{EL-Mechanisms for Bioactivities}

The underlying mechanisms responsible for the bioactivities of either naturally occurring lignans or their metabolites generally pertain to the antioxidant activity, the influence of lignans on hormone mechanisms, the estrogenic and anti-estrogenic effects, and an impact on gene expression and/or enzyme activity ${ }^{69}$. Based on numerous studies, the enterolignans, particularly EL, essentially account for these functions.

\subsubsection{Antioxidant Activity}

Oxidant by-products reactive oxygen species (ROS) such as superoxide $\left(\mathrm{O}_{2}^{-} \cdot\right)$, hydrogen peroxide $\left(\mathrm{H}_{2} \mathrm{O}_{2}\right)$, and hydroxyl radical $(\cdot \mathrm{OH})$ of normal metabolism often cause oxidative damage to DNA, proteins and lipids which give rise to degenerative diseases of aging such as cancers, CVD, immune-system decline and brain dysfunctions ${ }^{70}$. A number of studies provide evidence that plant lignans are capable of enhancing antioxidant defense mechanisms ${ }^{33,47,71-73}$. The antioxidant activities of the flaxseed lignan, SDG, and its mammalian lignans, enterodiol (ED) and enterolactone (EL) were evaluated in both lipid and aqueous in vitro model systems $^{33}$. All three lignans significantly $(\mathrm{p} \leq 0.05)$ inhibited linoleic acid peroxidation at both 10 and $100 \mu \mathrm{M}$ and DNA scissions in a concentration-dependent manner. In addition, SDG demonstrated the weakest activity at scavenging $\cdot \mathrm{OH}$ compared to ED and EL at both 10 and $100 \mu \mathrm{M}^{33}$. A study comparing the antioxidant activity of SECO, ED and EL against SDG and vitamin E showed that the antioxidant activity was highest with SECO and ED and lowest with vitamin E, and SECO, ED, and EL were respectively 3.82, 3.95, and 3.43 more potent than SDG, which may suggest the effectiveness of SDG in hypercholesterolemic atherosclerosis, diabetes, and endotoxic shock is generally due to its metabolites ${ }^{73}$.

\subsubsection{Estrogenic and Anti-estrogenic Effects}

The steroid hormone estrogen influences the growth, differentiation, and functioning of many target tissues including female and male reproductive systems such as mammary gland, uterus, vagina, ovary, testes, epididymis and prostate ${ }^{74,75}$. Given the widespread role for estrogen in human physiology, estrogen is involved in the development or progression of 
many diseases such as cancer (breast, ovarian, colorectal, prostate, endometrial), osteoporosis, neurodegenerative diseases, CVD and obesity, in some of which estrogen mediates its effects through binding the estrogen receptor $(E R)^{76}$. ER, existing in 2 main forms ER $\alpha$ and ER $\beta$, has distinct tissue expression patterns in both humans and rodents ${ }^{77}$. ER $\alpha$ and ER $\beta$ have high sequence homologies in the DNA binding domain (>90\%) and in the ligand binding domain $(\sim 60 \%)^{78}$. ER contains two transcriptional activation domains: the autonomous transcriptional activation domain AF-1, which is located at the N-terminus, and the lignan-dependent activation domain AF-2, located at the C-terminus; however, the primary sequence of AF-2 differs significantly between ER $\alpha$ and ER $\beta$. This gives rise to different agonist/antagonist features of various chemicals ${ }^{69}$.

Xenobiotics such as environmental or industrial chemicals and phytoestrogens that can interfere with the hormonal or endocrine system can mimic estrogen by binding to ER and induce (agonize) steroid response to cause estrogenic effects, or inhibit (antagonize) to prevent estrogenic activity, resulting in antiestrogenic effects ${ }^{79,80}$. The estrogenic or antiestrogenic effects at the cellular and molecular level are affected by many factors such as concentration dependency, receptor status, presence or absence of endogenous estrogens, and the type of target organ or cell ${ }^{81}$.

Lignans have received considerable attention as a source for phytoestrogens. Based on a study examining the mechanism to interfere with ER by which enterolignans exert estrogenic and/or antiestrogenic effects, it indicated that enterolignans had distinct properties for transactivation of $\mathrm{ER} \alpha$ and $\mathrm{ER} \beta$. ED, as estradiol (E2), induced ER $\alpha$ transcriptional activation through transactivation functions AF-1 and AF-2, while EL was less efficient in inducing AF-1 but acted predominantly through $\mathrm{AF}-2^{82}$.

The estrogenic effects of enterolignans were evaluated by some studies. In an early study, Sathyamoorphy found that EL at $1 \mu \mathrm{M}$ was able to produce a weak estrogenic response, while $\mathrm{ED}$ at $1 \mu \mathrm{M}$ was inactive. In addition, MCF-7 cell proliferation in the presence of $1 \mu \mathrm{M} \mathrm{EL}$ was $92 \%$ compared with cells treated with $0.1 \mathrm{nM} \mathrm{E2}$, whereas the addition of ED at the same concentration did not show growth stimulation ${ }^{83}$. In another study, E2 $(1 \mathrm{nM})$ and EL (0.5-2 $\mu \mathrm{M})$ separately stimulated the proliferation of MCF-7 cells, but their combination had no stimulatory effect compared to control ${ }^{84}$, which might result from the competition between EL 
and E2 on binding to the ER.

An antiestrogenic effect was also observed by some studies. Saarinen proposed that EL potently down regulated E2-stimulated angiogenic factors derived both from the stroma and the cancer cells ${ }^{46}$. Higher concentrations above $10 \mu \mathrm{M}$ of EL inhibited MCF-7 cells significantly $^{84}$. EL was evaluated for its effects on DNA synthesis in MCF-7 cells. Based on the analysis, EL was found to have a biphasic effect on DNA synthesis, exhibiting induction at $10-50 \mu \mathrm{M}$ (with a peak value of $210 \%$ at $10 \mu \mathrm{M}$ ) and inhibition at high concentrations, with an $\mathrm{IC}_{50}$ (half maximal inhibitory concentration) of $82.0 \mu \mathrm{M}^{85}$.

According to the above analysis, EL is likely essential for the estrogenic and antiestrogenic activities of flaxseed, suggesting the significance of the transformation from plant lignans to enterolignans by the intestinal bacteria. However, the serum concentration of EL in humans is normally $<10 \mu \mathrm{M}$ and most often $<1 \mu \mathrm{M}^{52}$. Therefore, a stimulatory effect on cell growth rather than inhibition is more likely to happen. The agonistic properties of EL might apply for conventional hormone replacement therapy in postmenopausal women. Nevertheless, the potential cell growth simulation and the ability to induce estrogen-responsive genes of EL need to be paid particular attention when used on hormone-dependent breast cancer patients ${ }^{69}$.

\subsubsection{Binding to Some Proteins (SHBG and SBP)}

Sex hormone binding globulin (SHBG) and sex steroid binding protein (SBP), glycoproteins in human plasma, have high affinity towards endogenous estrogens ${ }^{86}$. Lignans are proposed to potentially increase plasma SHBG and SBP levels, which then bind a large proportion of endogenous estrogens, thus decrease the free plasma estrogen and testosterone levels available to the cancer cells ${ }^{52,87}$. One study evaluated the correlation between intake of various fibers, lignan excretion and plasma levels of estrogens, free testosterone and SHBG in women and demonstrated a significant positive correlation between fiber intake and urinary excretion of lignans, and the concentration of plasma $\mathrm{SHBG}^{88}$. In addition, EL excretion correlated negatively with plasma percentage free estradiol and testosterone suggesting that fiber-rich foods containing lignans might stimulate SHBG and in this way to reduce levels of 
free hormone in plasma ${ }^{88}$. In an in vitro study EL stimulated the synthesis of SHBG by HepG2 liver cancer cells in culture acting synergistically with estradiol, which implied that lignans may affect uptake and metabolism of sex hormones by participating in the regulation of plasma SHBG levels ${ }^{89}$. Furthermore, Martin and coworkers studied the interactions of SBP and the lignans EL, ED, Nordihydroguaiaretic (NDGA) as well as isoflavonoid phytoestrogens (equol, diazein, and genistein). This researcher found that the phytoestrogens had different dose-dependent inhibitory effects on steroid binding by SBP, with relative efficiencies $\mathrm{EL} \geq \mathrm{NDGA}=$ equol $>$ genistein for displacing $\mathrm{E} 2$, but $\mathrm{ED}$ and diazein were much less active ${ }^{87}$.

\subsubsection{Influence on Enzyme Activity}

The mammalian lignans, especially EL, can inhibit several steroid metabolizing enzymes

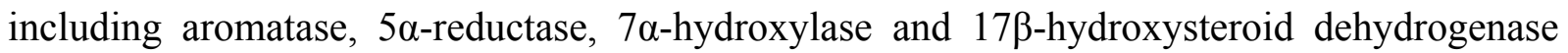
$(17 \beta-H S D)^{52}$. Aromatase, also named estrogen synthetase, is a cytochrome P450 enzyme responsible for catalyzing the conversion of androgens to estrogens ${ }^{90}$. EL was a moderate inhibitor of aromatase, while ED was a relative weak inhibitor in a human preadipose cell culture system $^{91,92}$. Such activity suggests that the high concentration of lignans in vegetarians, via inhibiting aromatase in peripheral and/or cancer cells and lowering estrogen levels, may play a protective role against estrogen-dependent cancers ${ }^{92}$.

The inhibition of $5 \alpha$-reductase and $17 \beta$-HSD by lignans in human genital skin fibroblast monolayers and homogenates, and in benign prostatic hyperplasia tissue homogenates was reported $^{93}$. EL inhibited both $5 \alpha-$ reductase and $17 \beta-$ HSD in genital skin fibroblasts, and EL was the most potent inhibitor of $5 \alpha$-reductase ${ }^{93}$. EL inhibited E2 production via inhibition of the $17 \beta$-HSD type 1 pathway $^{94}$. Additionally, the reduction in E2 production by EL was significantly related to a reduction in MCF-7 cell proliferation ${ }^{94}$.

\subsection{Pharmacokinetics of Plant and Mammalian lignans}

The pharmacokinetic properties of plant as well as mammalian lignans are rather complex and not thoroughly characterized. This section will summarize the absorption, 
metabolism, distribution and excretion of lignans, with emphasis on the conjugative glucuronidation of EL.

\subsubsection{Absorption}

\subsubsection{The absorbable form of lignans}

An oligomer composed of five SDG residues interconnected by four 3-hydroxy-3-methyl glutaric acid (HMGA) residues is identified as the main lignan of flaxseed ${ }^{95}$. SDG oligomers in the flaxseed extract are often hydrolyzed to break the ester linkages for the release of SDG and the glycosidic bonds for the release of $\mathrm{SECO}^{96}$. No studies specifically investigated whether SDG or the aglycone SECO is the absorbable form of lignans. Nevertheless, some indirect evidence suggests that the aglycone form SECO is primarily absorbed. Caco-2 cells derived from human colon adenocarcinoma were used as a model to investigate the bidirectional permeation (100 $\mu \mathrm{M}$ SDG, SECO, ED and EL) in our lab (manuscript under review). The results showed that the apical-to-basolateral and basolateral-to-apical permeation rates for SDG could not be calculated as SDG levels were below $50 \mathrm{ng} / \mathrm{mL}(0.0728 \mu \mathrm{M})$ under the level of quantification of our developed HPLC assay. However, permeation of SECO, ED and EL occurred in both of the apical-to-basolateral as well as basolateral-to-apical directions. In another study, the uptake of EL and ED by human colon epithelial cells was observed as the intracellular levels of conjugated EL and ED in HT29 cells rose immediately after starting the exposure, suggesting EL and ED are likely to permeate the colon epithelial cells and undergo phase II metabolism ${ }^{97}$.

Most of the lignan metabolites other than SDG were found in their conjugated forms in the portal vein after oral administration of flaxseed in $\operatorname{rats}^{98}$. An investigation showed that only in $5 \%$ of the 150 diphenolic fractions extracted from the urine of women plant lignans including LARI, isolariciresinol, MAT and SECO were identified, but SDG was not found in the urine ${ }^{99}$. When female rats were given a gavage of ${ }^{3} \mathrm{H}-\mathrm{SDG}(3.7 \mathrm{kBq} / \mathrm{g}$ body), ED, EL and SECO accounted for $75-80 \%$ of urine radioactivity, while SDG was not detected in the urine $^{100}$. The aforementioned data suggest that no measurable SDG levels in plasma and/or urine are found after flaxseed or pure SDG supplementation. Additionally, several distinct 
forms of aglycone were found after anaerobic incubation of SDG with a human fecal suspension, which demonstrates intestinal microflora is capable of transforming SDG into other aglycone forms ${ }^{101}$. Therefore, SDG is converted to its aglycone form, SECO, in the small intestine, which is subsequently absorbed or undergoes further conversion to the enterolignans.

\subsubsection{Pharmacokinetic parameters of lignans}

A pharmacokinetic study in rats following a single oral bolus dose of pure lignans (SDG, SECO, ED and EL was 40, 40, 10 and $5 \mathrm{mg} / \mathrm{kg}$, respectively) and an intravenous bolus dose of lignans (SDG, SECO, ED and EL was 20, 20, 5 and $1 \mathrm{mg} / \mathrm{kg}$, respectively) indicated that the serum concentration of SDG following oral administration was below the limit of quantification $(0.0728 \mu \mathrm{M})$ at all time points, whereas SDG level following intravenous dosing was detectable up to $4 \mathrm{~h}$ (manuscript under review). The concentrations of SECO in intravenous and oral serum samples were detected up to $8 \mathrm{~h}$ and $4 \mathrm{~h}$, respectively. The oral pharmacokinetic profile of SECO exhibited a very rapid absorption such that $\mathrm{C}_{\max }$ (the maximum drug concentration in the blood) was achieved at $5 \mathrm{~min}$. Quantifiable levels of ED were obtained up to $4 \mathrm{~h}$ post dose following both oral and intravenous administration. Besides, SECO exhibited the highest bioavailability of $\sim 25 \%$ in rats while SDG and ED showed poor oral bioavailability. Another pharmacokinetic analysis of SECO was performed in rats following a bolus intravenous injection (SECO was $20 \mathrm{mg} / \mathrm{kg})^{102}$. The systemic clearance $\left(\mathrm{Cl}_{\mathrm{s}}\right)$ for SECO was $7.3 \pm 1.1 \mathrm{~L} / \mathrm{h} \mathrm{kg}$, elimination rate constant $(\mathrm{k})$ was $0.15 \pm 0.01 \mathrm{~h}^{-1}$ and half life $\left(\mathrm{t}_{1 / 2}\right)$ was $4.5 \pm 0.4 \mathrm{~h}$. Volume of distribution $\left(\mathrm{V}_{\mathrm{d}}\right)$ for SECO was $47.4 \pm 10.9 \mathrm{~L} / \mathrm{kg}$ and area under the curve $\left(\mathrm{AUC}_{0-\infty}\right)$ was $2.79 \pm 0.41 \mathrm{~h} * \mu \mathrm{g} / \mathrm{mL}^{102}$.

In twelve healthy men and women enterolignans appeared in plasma 8-10 h after ingestion of a single dose of purified SDG $(1.31 \mu \mathrm{M} / \mathrm{kg} \text { body wt. })^{103}$. ED reached its maximum plasma concentration $14.8 \pm 5.1 \mathrm{~h}$ after ingestion of SDG, whereas EL reached its maximum $19.7 \pm 6.2 \mathrm{~h}$ after ingestion. The mean elimination half-life of ED ( $4.4 \pm 1.3 \mathrm{~h})$ was shorter than that of EL (12.6 $\pm 5.6 \mathrm{~h})$. The mean residence time for ED was $20.6 \pm 5.9 \mathrm{~h}$ and that for EL was $35.8 \pm 10.6 \mathrm{~h}^{103}$. In another seven volunteers EL plasma levels and urinary 
excretion were measured after the administration of a single dose of strawberries (500 $\mathrm{g})$ equivalent to $11.7 \mathrm{mg}$ of SECO and $0.61 \mathrm{mg}$ of MAT ${ }^{104}$. EL appeared in plasma $8 \mathrm{~h}$ after the post meal, and reached the maximum at $24 \mathrm{~h}$. Maximum EL excretion was observed in 25-36 h urine samples ${ }^{104}$.

\subsubsection{Conversion of lignans in human gastrointestinal tract}

In vitro conversion of plant lignans by human fecal flora ${ }^{101,105,106}$, germ-free rats $^{98}$ and humans taking antimicrobials ${ }^{107}$ demonstrates intestinal bacteria play a crucial role in the production of enterolignans. Intestinal bacteria metabolize plant lignans by a series of processes including deglycosylation, ring cleavage, demethylation, dehydroxylation and oxidation $^{69,101}$. Strains of Bacteroides and Clostridium genera deglycosylate SDG to form $\mathrm{SECO}^{108}$. It is suggested that the conversion occurs between terminal ileum and caecum in pigs $^{109}$. Similarly, Eeckhaut and his coworkers proposed SDG was only hydrolyzed into SECO through microbial action in the ascending colon ${ }^{110}$, which is supported by an early study in eight ileostomy patients having low plasma and urine lignan levels ${ }^{111}$. Subsequently, SECO is partially absorbed into systemic circulation and/or is biotransformed into mammalian lignans ${ }^{112,113}$. SECO undergoes dehydroxylation and demethylation catalyzing by strains of Ruminococcus products and Eggerthella lenta to yield ED, which can be further oxidized to form EL by Lactonifactor longoviformis ${ }^{108}$. As the minor lignan components in flaxseed, MAT, PINO and LARI can also be converted to EL by the bacterial flora through one and several additional steps, respectively ${ }^{42}$. Figure 2.3 outlines the bioconversion from plant lignans to mammalian lignans by the intestinal microflora in the gastrointestinal tract. Apparently, diverse bacteria communities are favorable to the transformation of plant lignans to mammalian lignans; thus, the variation of intestinal bacterial may result in large inter-individual difference on EL levels. 


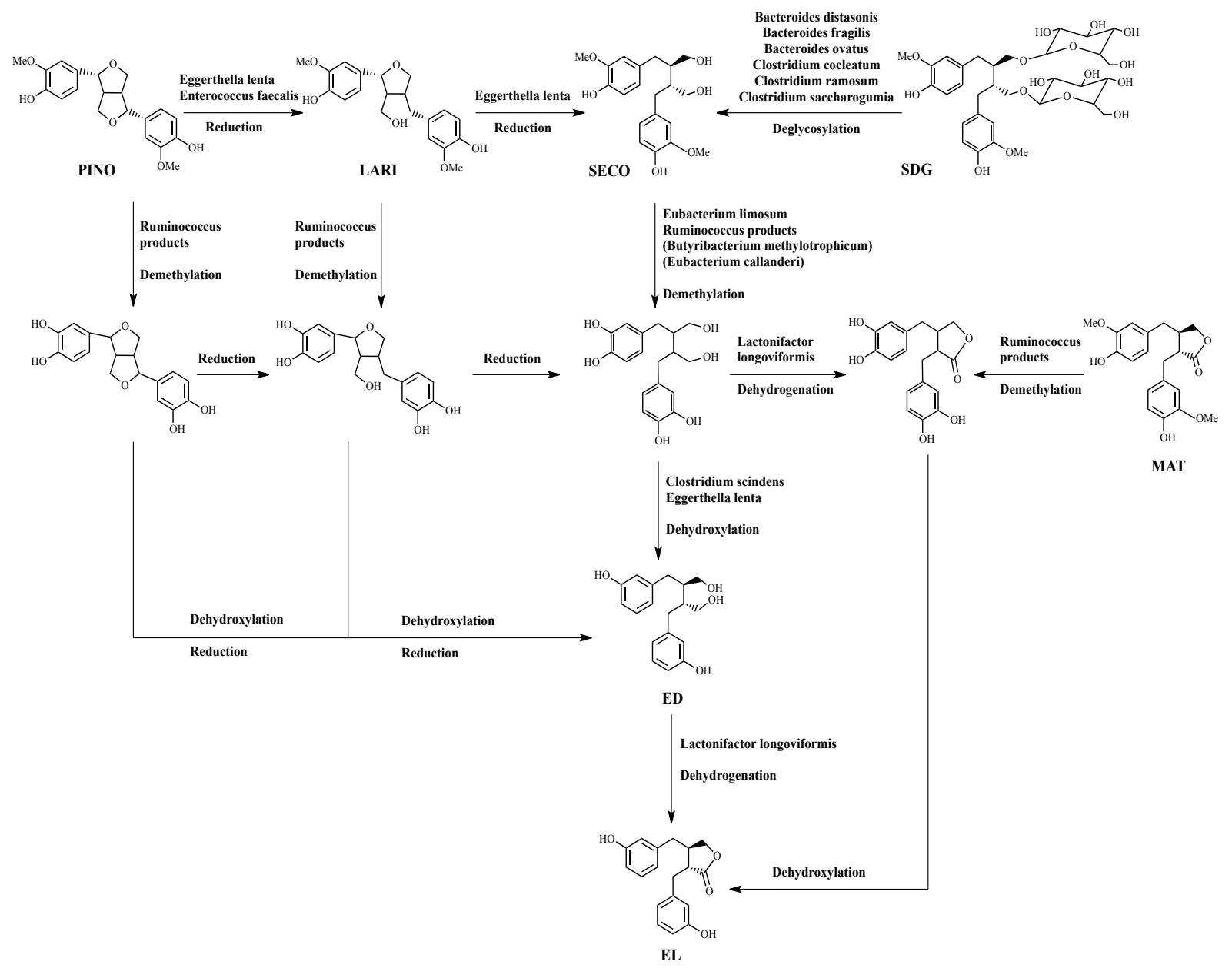

Figure 2.3 Diagram of the intestinal microflora mediated conversion of lignans in human gastrointestinal tract. 


\subsubsection{Metabolism}

Once mammalian lignans are absorbed, they undergo primarily phase II metabolism prior to systemic circulation, with the predominant formation of conjugative metabolites, particularly EL glucuronides ${ }^{97,114,115}$.

\subsubsection{Oxidative metabolism}

Oxidative metabolism of enterolignans is principally mediated by hepatic P450 enzymes. Human and rat hepatic microsomes biotransform EL to oxidative metabolites with an additional hydroxyl group either at the aromatic or aliphatic moiety ${ }^{116}$. A study evaluating the metabolic fate of EL and ED by ingesting flaxseed to humans showed that monohydroxylated EL and ED metabolites were identified at the para- and ortho-positions on either aromatic ring, which indicated the lignan was oxidatively metabolized in vivo ${ }^{117}$. This is in agreement with the results found in rats where several hydroxylated metabolites of EL and ED were found in bile and urine after feeding a diet containing 5\% flaxseed $^{114}$ (Figure 2.4 and Figure 2.5). Nevertheless, the phase I metabolites of enterolignans represent a minor percent when compared with phase II metabolites ${ }^{115,117}$.

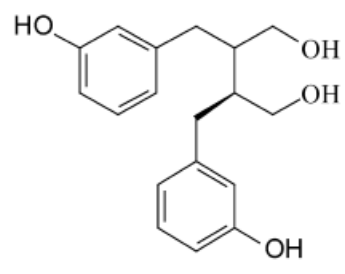

$$
\text { Enterodiol }
$$
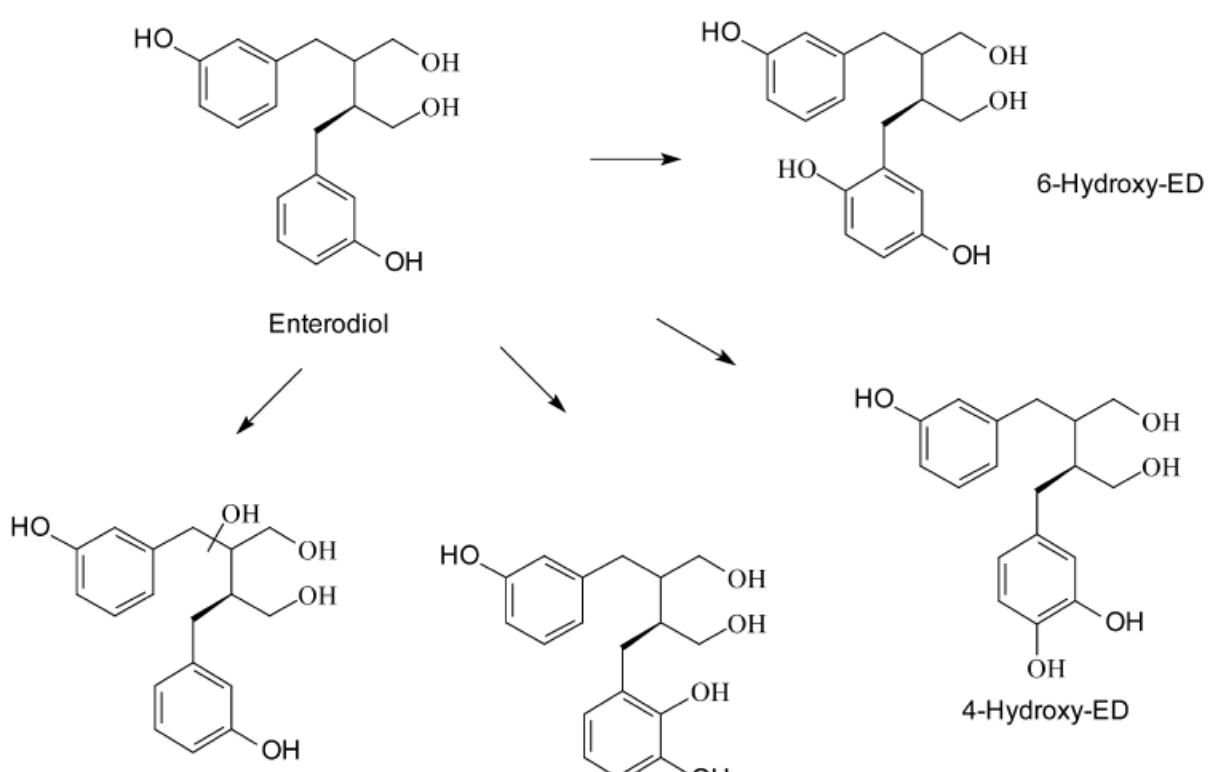

Aliphatic Hydroxylation Products of ED

$\mathrm{HO}$<smiles>Cc1cccc(CC(CO)C(CO)CO)c1</smiles>

2-Hydroxy-ED

Figure 2.4 Oxidative metabolites of ED (Adapted from Alister D. Muir ${ }^{118}$ ) 


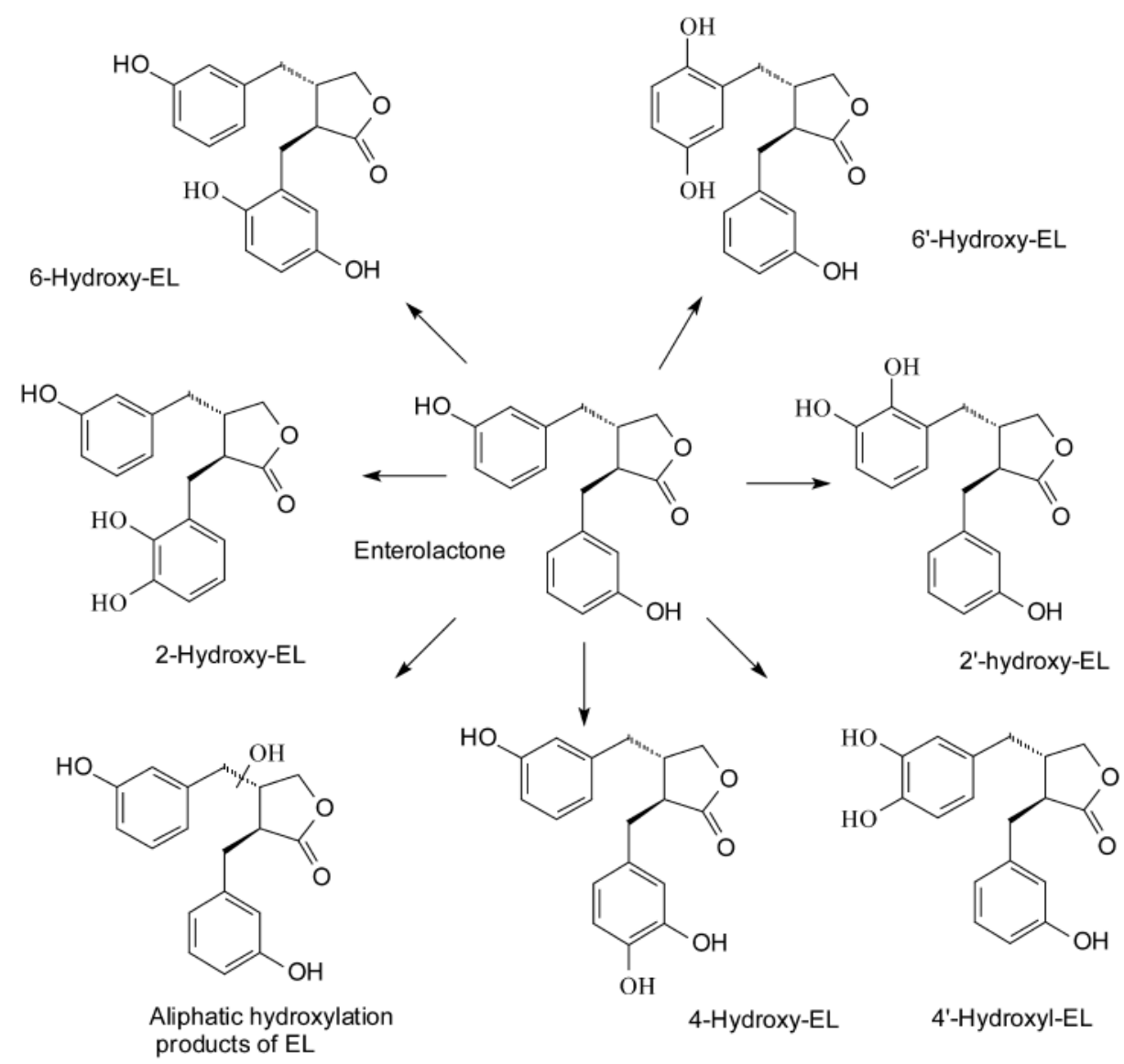

Figure 2.5 Oxidative metabolites of EL (Adapted from Alister D. Muir ${ }^{118}$ )

\subsubsection{Conjugative metabolism}

\subsection{UDP-glucuronosyltransferases}

The UDP-glucuronosyltransferases (UGT) are a superfamily of endoplasmic reticulum membrane-bound enzymes, utilizing UDP-glucuronic acid as a cosubstrate for the formation of glucuronides from substrates such as steroids, bile acids, hormones and thousands of xenobiotics that include drugs, environmental toxicants and carcinogens, the process of which is known as glucuronidation ${ }^{119}$. Glucuronidation transforms lipophilic substrates into hydrophilic glucuronides, more water-soluble compounds that can be subsequently eliminated through the bile and urine. Thus, the glucuronidation pathway is primarily regarded as a detoxification reaction $^{120}$. The highest UGT activities frequently occur in the microsomal fractions extracted from the liver, kidney and intestine, and various factors such as age, gender, hormonal status, genetic factors as well as environmental exposures will exert great influence 
on the exact composition of UGT in tissues ${ }^{120}$.

In humans, four UGT families have been identified including UGT1, UGT2, UGT3, UGT8, in which UGT1 and UGT2 mainly use UDP-glucuronic acid to glucuronidate endogenous substances and xenobiotics including lignans, flavonoids and isoflavones that are not accessible for UGT3 and UGT8 families ${ }^{121}$. Up to now, UGT1 family includes 9 functional isoforms in humans, and 7 in rat; UGT2 include 6 members in humans and 7 in rat $^{119-121}$. Since the expression of UGT in the same and/or different organs between humans and rats is different, species difference in glucuronidation can be expected.

\subsection{Enterolignan glucuronidation}

ED and EL undergo hepatic phase II reactions, primarily glucuronidation, with minor contributions to phase II metabolism by sulfotransferases ${ }^{122,123}$. ED and EL were incubated with rabbit liver microsomal UGT and the lignan glucuronides were purified. The monoglucuronide conjugates of each lignan were confirmed, which indicated the hepatic microsomal UGT system conjugated EL to either phenolic hydroxyls and ED to either non-phenolic or phenolic hydroxyl groups ${ }^{124}$ (Figure 2.6). Dean and coworkers also substantiated that EL underwent extensive glucuronidation with rhesus liver microsomes to form O-glucuronides at both phenolic hydroxyl groups ${ }^{115}$ (Figure 2.7). Additionally, incubations of EL with human and rhesus hepatocytes resulted in a large percent of glucuronic acid conjugates, with minor amounts of the sulfate conjugates and monohydroxylated products $^{115}$.

In addition to the hepatic glucuronidation, several studies have demonstrated the intestinal glucuronidation of enterolignans. Human colon epithelial cells (HT29 and Caco-2) can take up, metabolize and excrete the enterolignans EL and ED, and these cell lines produce conjugation products, which exist as EL monoglucuronide, EL sulfate and ED glucuronide. Moreover, more than $90 \%$ of the EL in the medium was present in its conjugated form within $10 \mathrm{~h}$, however in vivo a significant amount of EL is probably conjugated by the intestinal cells before entering the circulation ${ }^{97}$. The extent of glucuronidation and sulfation of lignans (SDG, SECO, ED and EL) in the Caco-2 monolayer was also assessed in our own lab (manuscript 
under review). Limited conjugation of SDG was observed up to $48 \mathrm{~h}$ of incubation with Caco-2 cells, but SECO, ED and EL exhibited significant conjugation. Hydrolysis of cell lysate and supernatant media with $\beta$-glucuronidase/sulfatase enzyme showed less than 3\% SDG conjugation. SECO and ED presented $95 \%$ and $90 \%$ conjugation, respectively, while EL was completely conjugated within $8 \mathrm{~h}$. This implies that lignans undergo extensive and significant first-pass metabolism prior to systemic circulation.

Subsequently, conjugated lignans are excreted back to the digestive tract via bile, reabsorbed from the intestine, and eventually are excreted in the urine as glucuronide conjugates, a process known as enterohepatic circulation ${ }^{98}$ (Figure 2.8). This process enhances the exposure of enterolignans to intestine and liver, resulting in further substantial conjugation reactions.

Most of the ED and EL detected in the portal vein of rats were present principally as glucuronide conjugates ${ }^{98}$. In a plasma analysis of enterolignans in 27 pre- and postmenopausal women approximately $80 \%$ total enterolignans were present as monoglucuronides, with minor a percent of diglucuronides and sulfoglucuronides ${ }^{123}$. The excretion of enterolignans in urine and bile was largely as glucuronides, while mono- and disulphates were also found ${ }^{98}$. In human urine, $92 \%$ of ED and $98 \%$ of EL in the glucuronide fraction were observed, in which 84.5\% monoglucuronide of ED and 94\% monoglucuronide of EL occurred in the glucuronide fractions $^{125}$.

Thus, most of the absorbed dose of lignans predominantly exists as monoglucuronide conjugates. These findings raise some important questions regarding the bioactive lignan form, i.e. whether the plant lignans or their conjugates, or the mammalian lignans or their conjugates mediate the positive health benefits of flaxseed consumption. Traditionally, glucuronidation is considered as a reaction that results in the loss of pharmacological activity of xenobiotics and rapid excretion. However, several glucuronide conjugates are known to possess biological effects. For instance, a widely used opioid analgesic 6-O-glucuronide of morphine contributes to the pharmacological activity in addition to the parent compounds ${ }^{120}$. Given the high concentrations of enterolignans glucuronides, the pharmacological activity of these glucuronides needs further study. 


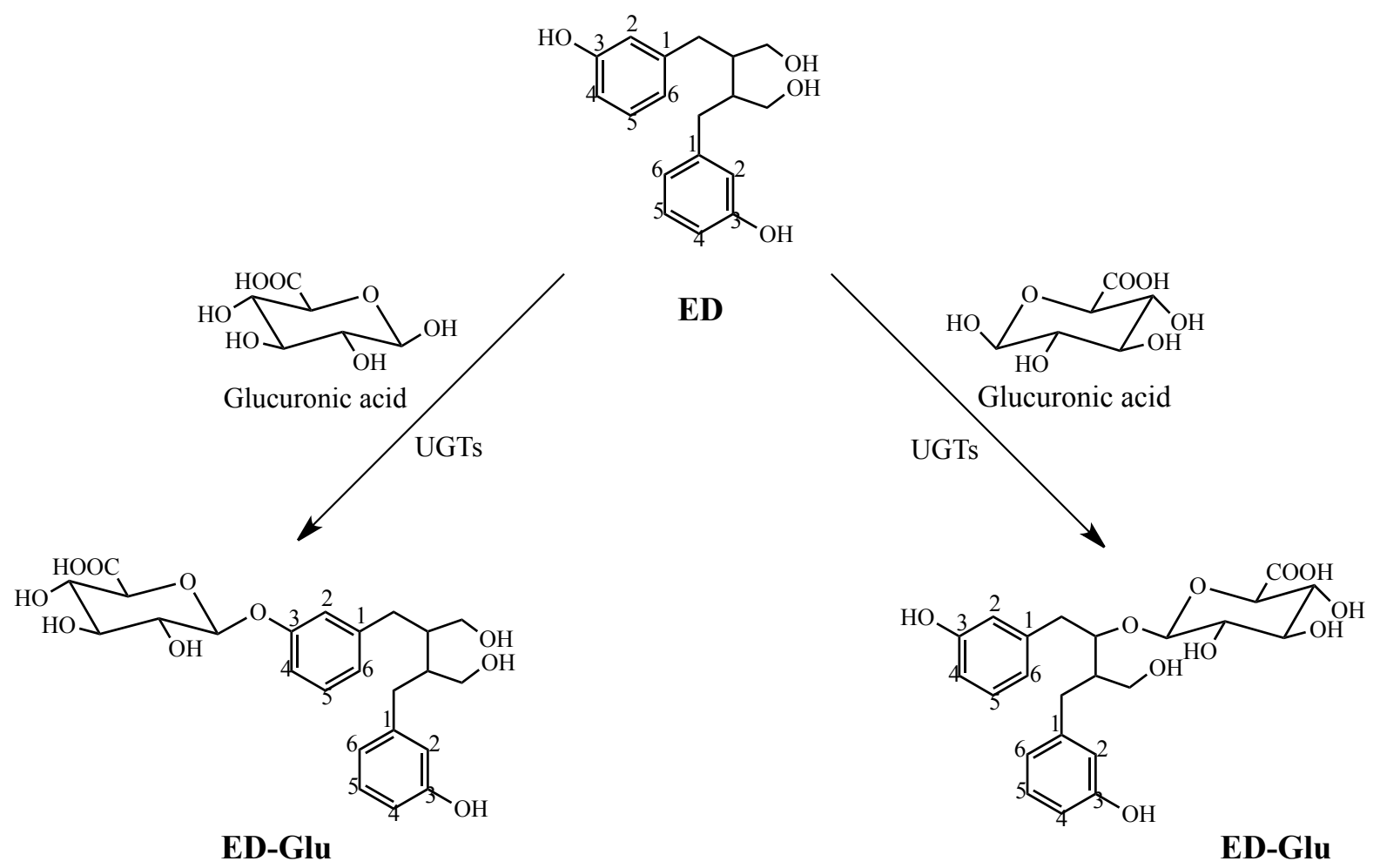

Figure 2.6 Conjugative glucuronidation metabolites of ED

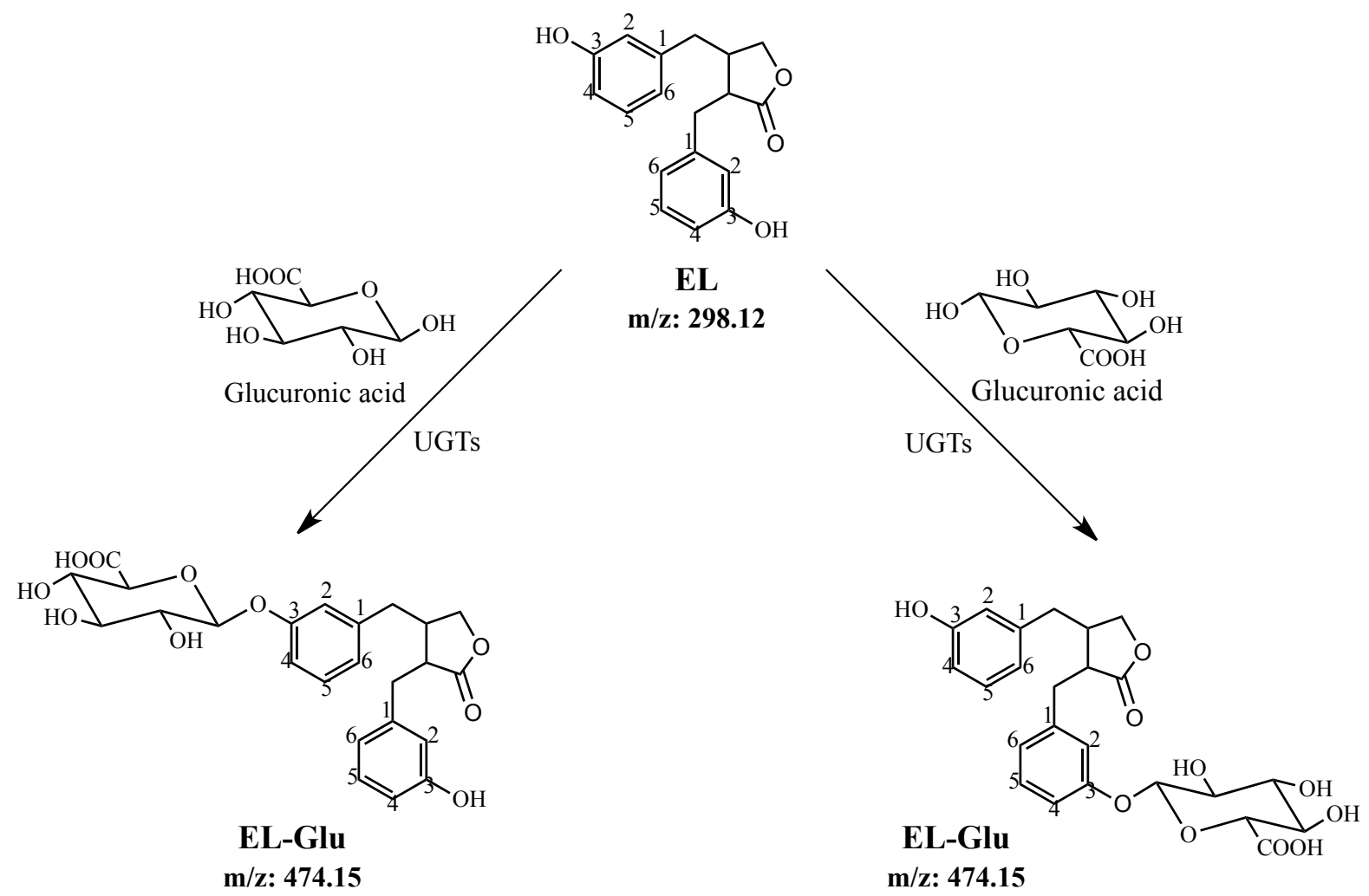

Figure 2.7 Conjugative glucuronidation metabolites of EL 


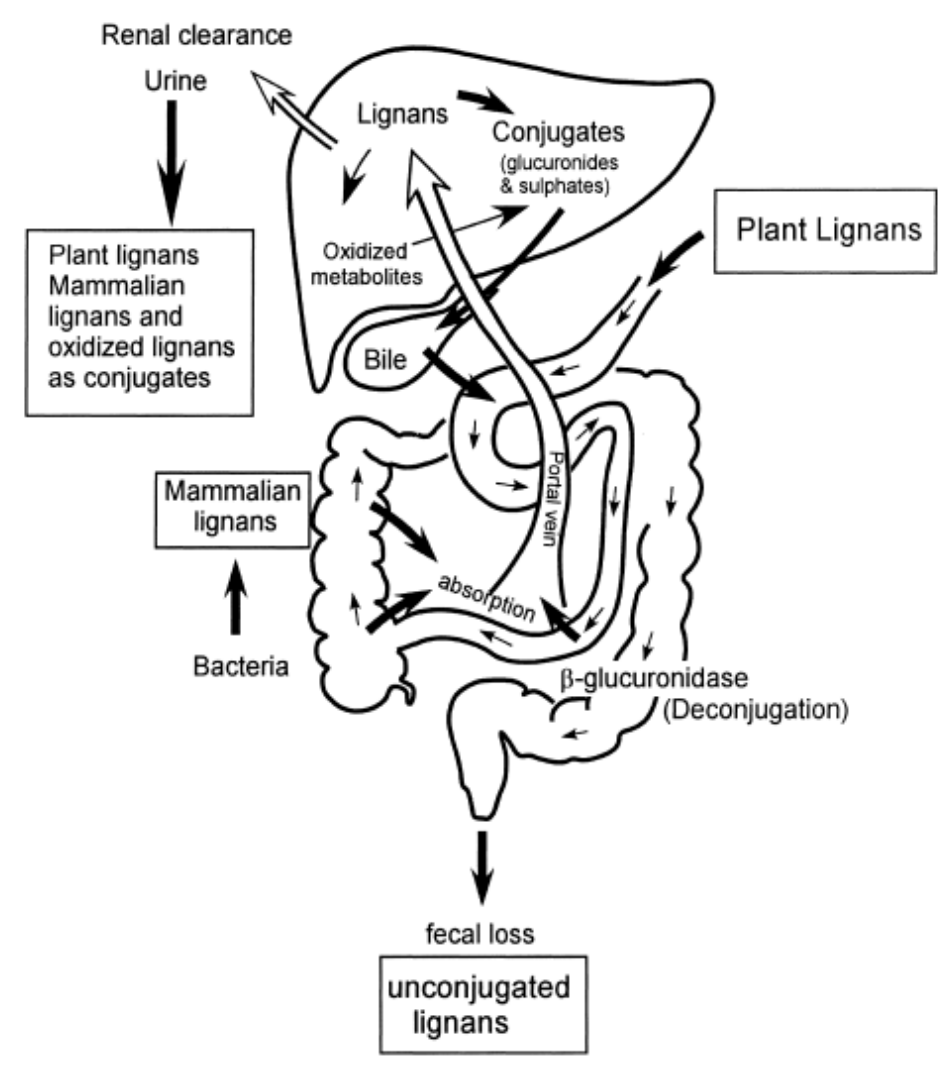

Figure 2.8 The enterohepatic circulation of plant lignans, their colonic and liver metabolites (Adapted from Setchell 1982 $2^{126}$ )

\subsubsection{Distribution}

Despite the biological forms of lignans that are beneficial to human health, the efficient delivery of drugs from the site of administration to the target organs, tissues or receptors is required. However, little is known about the lignan distribution and the concentrations in the tissues, which are essential to understand their pharmacological effects and underlying mechanisms of pharmacological action. Some general information observed in tissue distribution studies is presented below.

In rats, a dose-related increase in the tissue distribution of ED and EL was observed in rat liver, testes, prostate and lung after administration of SDG, where the liver and prostate achieved the highest concentrations of ED (1.844 and $1.899 \mathrm{pmol} / \mathrm{mg}$, respectively) of all tissues examined at the $60 \mathrm{mg} / \mathrm{kg}$ SDG, a significant increase over the levels obtained in the controls and $15 \mathrm{mg} / \mathrm{kg}$ dose group ${ }^{127}$. In addition, lung exhibited a significant 2.8 -fold increase 
in EL concentration with the dosage of SDG increase from 15 to $60 \mathrm{mg} / \mathrm{kg}^{127}$. Lignans, but only in the form of EL, were also detected in the tissue of colon, liver, breast and brain at a much higher level with rye than wheat when high-fiber wheat and high-fiber rye bread administered to pigs for 58-67 $\mathrm{d}^{112}$. Tissue accumulation of lignans in human prostate and/or breast cyst fluids was observed in several studies, suggesting an anticarcinogenic effect towards prostate and breast cancer. In the semen of 6 men, EL existed in both conjugated and unconjugated forms at the mean concentration of $183 \mathrm{nM}$, which was much greater than 73 $\mathrm{nM}$ in the blood $^{128}$. In the semen of 4 bulls, the mean concentration of total conjugated and unconjugated EL was $3200 \mathrm{nM}$, an order of magnitude higher than that was in the blood (228 $\mathrm{nM})^{128}$. In 191 women, the median intracystic level of EL (63 nM) was approximate four times higher than the median value of serum EL $(17 \mathrm{nM})^{129}$. Therefore, the accumulation of EL in prostate and/or breast cyst fluids might be, in part, responsible for decreasing the prostate and/or breast cancer risk.

Chronic or prolonged administration of SDG might alter lignan distribution. ${ }^{3} \mathrm{H}-\mathrm{SDG}$ disposition in female rats with acute or chronic SDG treatment over $48 \mathrm{~h}(3.7 \mathrm{kBq} / \mathrm{g}$ body weight $3 \mathrm{H}-\mathrm{SDG}$ versus $1.5 \mathrm{mg}$ unlabeled SDG/d) was evaluated by the Thompson group. The results found that tissue radioactivity was highest (by 0.5 to 176 -fold) in the cecum, and levels in the liver, kidney and uterus were 0.2 - to 7.5 -fold higher than in other nongastrointestinal tissues ${ }^{130}$. Also, cecal content, liver and adipose radioactivity were one-to-threefold greater in rats with chronic SDG exposure as compared with acute exposure ${ }^{130}$. In a later study by the same research group ${ }^{3} \mathrm{H}-\mathrm{SDG}$ administration $(3.7 \mathrm{kBq} / \mathrm{g}$ body weight) resulted in an increased radioactivity in all tissues (liver, kidneys, bladder, spleen, lungs, brain, thymus, heart, muscle, adipose, mammary gland, ovaries, vagina, uterus, testis, seminal vesicles, coagulating glands and ventral prostate) examined in both male and female rats, and the levels were further increased after prolonged SDG exposure, while liver contained the majority of the tissue lignans (48-56\%) in both sexes after $1 \mathrm{~d}$ or $7 \mathrm{~d}$ exposure regimens ${ }^{131}$. After prolonged exposure, females had higher lignan concentrations in heart and thymus, demonstrating sex-related differences in lignan tissue distribution ${ }^{131}$. Hence, the foregoing data suggest lignans mainly accumulate in the liver, prostate, kidney and intestine. However, it is noteworthy that the tissue distribution of lignans was mostly investigated through animal 
models such as rats; thus, careful attention should be paid when extrapolating the distribution pattern from animals to humans.

Since only the unbound form of a compound can exert an effect, the extent to which lignans bind to serum protein is of importance. However, limited information about the plasma protein binding data of flaxseed lignans is available in the literature. SDG, SECO, ED and EL (each $50 \mu \mathrm{g} / \mathrm{mL}$ ) were evaluated for their serum protein binding capacity in rat serum by our developed HPLC method (manuscript under review). The results showed that SDG was not bound to serum protein, while SECO, ED and EL displayed increasing serum protein binding characteristics in an ascending order. This similar trend of the binding affinities of SECO, ED and EL was also observed with steroid hormone binding globulins (SHBG) that SECO and EL had higher binding affinity than $\mathrm{ED}^{132}$. Besides, SECO and EL displaced $60 \pm$ $7 \%$ and $55 \pm 7 \%$ of ${ }^{3} \mathrm{H}$-dihydrotestosterone (DHT) from the binding site, respectively, whereas ED exhibited only $16 \pm 6 \%{ }^{133}$.

\subsubsection{Excretion}

Urinary excretion is one of the primary routes of excretion of lignan metabolites. The excretion of enterolignans in urine and bile were mainly as glucuronides, while small amounts of mono- and disulphates were also found ${ }^{98}$. In human urine, $92 \%$ of ED and $98 \%$ of EL in the glucuronide fractions were observed ${ }^{125}$. A very small percent of plant lignans was also excreted in the urine, which might result from an insufficient metabolic capacity of intestinal bacteria $^{99}$. The urinary excretion of EL varied from 1 to $4.2 \mu \mathrm{M} / 24 \mathrm{~h}^{134,135}$, and the urinary excretion of enterolignans is significantly related to lignan intake. Rickard and coworkers conducted a study in rats fed ground flaxseed $(2.5,5$ or $10 \mathrm{~g} / 100 \mathrm{~g})$ or SDG $(1.1,2.2$ or 4.4 $\mathrm{mM}$ ), and found that urinary lignan excretion increased linearly with doses from 0-5\% flaxseed and 0-2.2 mM SDG/ $\mathrm{d}^{136}$. In postmenopausal women consuming 5 or $10 \mathrm{~g}$ of ground flaxseed/d, urinary excretion of ED was increased by 1,009 and $2867 \mathrm{nM} / \mathrm{d}$, while urinary excretion of EL was enhanced by 21,242 and $52,826 \mathrm{nM} / \mathrm{d}^{137}$.

Fecal excretion is another major route of excretion of lignan metabolites. Fecal excretion of the lignans ED, EL and MAT increased significantly with flax consumption, from 80 to 
2560,640 to 10300 and 7.33 to $11.9 \mathrm{nM} / \mathrm{d}^{138}$. Excretion in feces of pigs, predominantly in the form of EL, represented $46 \%$ of the intake when pigs were fed rye diet containing high percent of lignans ${ }^{139}$. This is somewhat higher than that which was found in humans, where $34-35 \%$ (mean) of the mammalian lignans were excreted through the fecal route ${ }^{139}$. When rats were given a single gavage of ${ }^{3} \mathrm{H}-\mathrm{SDG},>80 \%$ of the recovered dose was excreted in both feces ( $>$ $50 \%$ ) and urine (28-32\%) after $48 \mathrm{~h}^{130}$. The high fecal excretion of enterolignans may be due to the incomplete absorption or enterohepatic circulation.

\subsection{Bioavailability of EL}

The proposed health benefits of lignans have promoted its consumption by individuals suffering from various chronic diseases. However, systemic levels of EL are highly variable in the population. Several factors are crucially responsible for the varying circulating EL concentrations, which are discussed below.

\subsubsection{Dietary and Lifestyle Determinants}

Dietary and lifestyle determinants are important factors that influence circulating EL concentrations. Dietary components such as whole-grain products, vegetables, fiber and coffee showed positive associations with plasma EL concentration, while negative associations were observed among body mass index (BMI), smoking and frequency of bowel movements with plasma EL concentration ${ }^{140-142}$.

\subsubsection{Interindividual Variation of Gut Microflora}

Interindividual variations in bacteria lead to significant differences of lignan metabolism in the population, which can be separated into high, moderate and low mammalian lignan producers $^{143}$. In vitro SECO incubation with human fecal samples showed that higher proportions of Peptostreptococcus products and related species, as well as bacteria belonging to the Atopobium group were typical for moderate to high concentrations of EL-producing communities $^{106}$, while ED production correlated negatively with Clostridium coccoides-Eubacterium rectale counts ${ }^{143}$. Furthermore, since gut bacteria are critical in the 
formation of EL from plant precursors, factors that affect lignan-converting bacteria will potentially influence the absorbable level of EL. As enterolignans precursors usually occur in fiber-rich foods, dietary fiber might play a profound role on the number and diversity of bacteria that inhabit the large intestine ${ }^{144}$. The presence of fermentable dietary fiber in lignan-rich foods is likely to increase microfloral bacteria level, resulting in an enhanced level of EL. Another influential factor on intestinal bacteria is antibiotics. The impact of antibiotics on EL concentration has been reported by several studies, which suggested that the concentration of EL in blood was reduced by antibiotics among those who had used antibiotics during the preceding year ${ }^{107,145}$. Consequently, interindividual variation in the density and diversity of gut microflora may significantly contribute to varying levels of EL.

\subsubsection{First-pass Glucuronidation}

Another critical factor causing the variable systemic concentrations of EL is its low and varying bioavailability owing to the extensive first-pass glucuronidation. In human plasma and urine, the principal conjugates of EL were mainly monoglucuronides, whereas very low levels of free EL were found ${ }^{122,123,125}$. High circulating levels of EL glucuronide with concomitant low levels of EL suggest a significant first-pass effect by glucuronidation. Glucuronidation occurs both in hepatocytes and enterocytes. Extensive glucuronidation results in variable and significant reductions in the oral bioavailability of EL, which may limit the biological properties of EL. However, the exact contributions from liver and intestine are poorly understood.

Hence, a fundamental understanding of EL glucuronidation in the intestine and liver in vitro is essential to provide critical information regarding the oral absorption properties of EL, and also possibly to infer the potential for interactions. The in vitro evaluations of EL glucuronidation are obtained by the determination of intrinsic clearance $\left(\mathrm{CL}_{\mathrm{int}}\right)$, which directly measures the efficacy of enzymes to metabolize substrates ${ }^{146}$. Therefore, the in vitro evaluations of EL glucuronidation can provide a great understanding as to what extent EL undergoes intestinal and hepatic glucuronidation, which conjugation metabolites would be produced, and also the contributions from intestine and liver to EL glucuronidation. 


\subsection{Model System}

\subsubsection{Microsomes}

Two basic strategies are often used for in vitro investigation of the metabolic profile of a drug: incubation with subcellular fractions, for example, microsomes, and incubation with differentiated cellular models such as primary cultures, encapsulated hepatocytes or tissue slices $^{147}$. With intact cells, the plasma membrane, metabolic pathways, levels of physiological cofactors and coenzymes and active gene expression are reasonably well maintained for several hours/days in culture, which makes hepatocytes the closest model for in vivo studies ${ }^{147}$. However, microsomes are often the simple, affordable and best-characterized in vitro model, allowing an easy comparison of the metabolic profile of a compound across animal species $^{147,148}$. Microsomes are easily prepared from tissues by homogenization and centrifugation, which contain almost only P450 and UGT enzymes. Microsomes can be stored at $-80^{\circ} \mathrm{C}$ for years with little or no loss of enzyme activities. As well, microsomes from different animal species are commercially available and are well standardized. The main drawback of microsomes is that the results obtained in vitro might be different from those obtained in vivo due to the enriched enzymes in microsomal fractions and the absence of other competing enzymes, but some equations exist to extrapolate in vitro data to in vivo pharmacokinetics $^{148}$.

\subsubsection{Intrinsic clearance}

Intrinsic clearance $\left(\mathrm{CL}_{\text {int }}\right)$ is the ability of each organ to remove drugs from the blood, which is independent of physiological factors such as blood flow and protein binding ${ }^{149} \cdot \mathrm{CL}_{\text {int }}$ directly measures the efficacy of enzyme to metabolize substrate ${ }^{146}$. Hence, determination of $\mathrm{CL}_{\text {int }}$ in vitro will identify the contribution of the enzymes of each organ to the metabolism of the xenobiotics in vivo. Since $\mathrm{CL}_{\text {int }}$ is the cornerstone to in vitro-in vivo extrapolation of metabolic data ${ }^{146}$, the comparison of intestinal $\mathrm{CL}_{\text {int }}$ with hepatic $\mathrm{CL}_{\text {int }}$ by the enzyme kinetic studies in vitro will, to a large degree, inform us of the first-pass glucuronidation of EL as well as the contribution of each organ to the first-pass metabolism of EL in vivo. 


\subsubsection{Human versus Rat Models}

Although humans are the most relevant system for evaluation, animal models are commonly used in the preclinical development of new drugs to predict the metabolic profile of new compounds in human. In comparison with other animal models, rat offers many advantages and is an excellent model for human diseases because of the well-characterized physiology that is more like the corresponding human conditions ${ }^{150}$. A volume of data has developed and numerous experimental protocols are well established, which enables an easier way to obtain information to design experiments, make comparisons between studies, and to extrapolate from in vitro to in vivo and from animals to humans. Furthermore, rat is frequently used as an experimental model to explore and evaluate the pharmacokinetic activities of lignans. Thus, conducting intestinal and hepatic glucuronidation of EL in rats is important to human health. The results will be considerably valuable for further future study and extrapolating to humans. 


\subsection{Hypothesis and Objectives}

SDG and its metabolites, especially EL, have received increasing interest for their putative health benefits. Once generated in the intestine, EL permeates the gastrointestinal barrier and undergoes conjugation with principally glucuronic acid before entering the systemic circulation. In human plasma and urine, the predominant conjugates of EL are mainly glucuronides, while very low levels of free EL were found. This suggests a significant first-pass glucuronidation occurring both in hepatocytes and enterocytes, which, to a large extent, results in variable and significant reductions in the oral bioavailability of EL. However, the exact contributions from liver and intestine are poorly understood. Hence, the purpose of the study is to provide an estimate of the relative contribution of the intestine and liver to presystemic glucuronidation of EL, which will provide a fundamental understanding regarding the oral absorption properties of EL. In vitro enzyme kinetic studies using intestinal and hepatic microsomal fractions from human and rat with determination of $\mathrm{CL}_{\text {int }}$ allow the comparison of differences of EL glucuronidation between different organs and species. The aims of this project are accomplished by the following hypothesis and objectives:

\subsubsection{Hypothesis}

The intrinsic clearance $\left(\mathrm{CL}_{\text {int }}\right)$ values from rat and human liver microsomes are greater than the values from intestinal microsomes.

\subsubsection{Objective 1}

Enzymatically generate EL glucuronides in rat liver microsomes, then purify and verify the glucuronides.

\subsubsection{Objective 2}

Validate the existing HPLC assay to allow for the quantitative determination of EL.

\subsubsection{Objective 3}

Conduct enzyme kinetic studies using rat and human hepatic and intestinal microsomal 
fractions to determine and compare $\mathrm{CL}_{\text {int }}$ of EL glucuronidation from each organ system. 


\section{MATERIALS AND METHODS}

\subsection{Chemicals}

Enterolactone (EL), uridine 5'-diphosphoglucuronic acid trisodium salt, D-saccharic acid 1,4-lactone monohydrate, Trizma base, bovine serum albumin (BSA) and internal standard (umbelliferone) were obtained from Sigma-Aldrich Canada Ltd (Oakville, ON). A MilliQ Synthesis (Millipore, Bedford, MA) Water Purification system provided purified deionized water. All other chemicals used were analytical grade. Human liver and intestinal microsomes were purchased from XenoTech, LLC (Lenexa, KS).

\subsection{Experiment one: EL Glucuronide Synthesis and Characterization}

\subsubsection{Preparation of Rat Liver and Intestinal Microsomes}

Male Wistar rats ( $\mathrm{N}=6$, weight range 250-300 g and age range 7-9 weeks) were obtained from the Animal Resources Centre (ARC), University of Saskatchewan. This work was approved by the University of Saskatchewan's Animal Research Ethics Board, and adhered to the Canadian Council on Animal Care guidelines for humane animal use.

\subsubsection{Preparation of rat liver microsomes}

Rat liver and intestinal microsomes were prepared according to established protocols in our lab. Rat liver was removed promptly following isoflurane anesthesia of the rat and rinsed twice in ice water to remove excess blood. The liver was immediately immersed into liquid nitrogen and stored at $-80^{\circ} \mathrm{C}$ until preparation. Three grams of rat liver from three individuals were homogenized in $12 \mathrm{~mL}$ buffer ( $\mathrm{pH}$ 7.4) containing $50 \mathrm{mM}$ Tris buffer, $150 \mathrm{mM} \mathrm{KCl}, 0.1$ $\mathrm{mM}$ dithiothreitol, $1 \mathrm{mM}$ ethylenediamine-tetraacetic acid (EDTA), 20\% glycerol, $0.1 \mathrm{mM}$ phenylmethylsulfonylflouride (PMSF) and double distilled water $\left(\mathrm{ddH}_{2} \mathrm{O}\right)$. The liver homogenate was then ultracentrifuged at $9,184 \times \mathrm{g}$ for $30 \mathrm{~min}$. The supernatant was transferred and ultracentrifuged at 112,504 $\times \mathrm{g}$ for $30 \mathrm{~min}$. The microsomal pellet was washed with $150 \mathrm{mM} \mathrm{KCl}$ in $\mathrm{ddH}_{2} \mathrm{O}$, and then ultracentrifuged at $112,504 \times \mathrm{g}$ for another $30 \mathrm{~min}$. Microsomal pellets were resuspended in $0.25 \mathrm{M}$ sucrose solution. The rat liver microsomes 
were stored at $-80^{\circ} \mathrm{C}$. The total protein concentration was determined by BioRad RC DC Protein Assay Kit (Bio-Rad Laboratories Ltd., Mississauga, ON) using bovine serum albumin (BSA) as a standard.

\subsubsection{Preparation of rat intestinal microsomes}

To compare the metabolic activities of different sections of rat small intestine, intestinal microsomes extracted from duodenum, jejunum and colon were prepared. Tissue consisting of the proximal duodenum, jejunum and colon were removed from anaesthetized rats $(n=2)$. The intestinal sections were instantly placed in ice-cold buffer solution $\mathrm{A}(\mathrm{KCl} 1.5 \mathrm{mM}, \mathrm{NaCl} 96$ $\mathrm{mM}$, sodium citrate $27 \mathrm{mM}, \mathrm{KH}_{2} \mathrm{PO} 48 \mathrm{mM}, \mathrm{Na}_{2} \mathrm{HPO} 45.6 \mathrm{mM}$, pH to 7.4$)^{151}$ to flush out intestinal contents. The remaining intestinal sections were dissected longitudinally and spreaded on an ice-cold glass plate with the mucosal side upwards. The mucosa was then gently scraped using a microscope slide. The scraped mucosa was homogenized in solution B

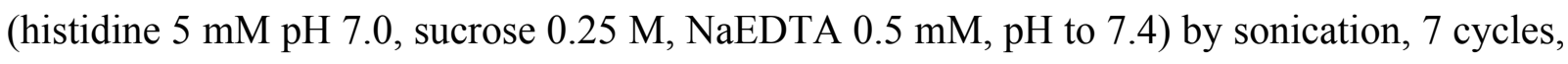
10 seconds each (wattage $=4$ ). The homogenate was then centrifuged at $15,000 \times \mathrm{g}$ for $10 \mathrm{~min}$. The supernatant was collected, and $1.25 \mathrm{~mL}$ of $52 \mathrm{mM} \mathrm{CaCl}_{2}$ for each $7 \mathrm{~mL}$ supernatant was added. The tubes containing microsomes were gently shaken for $10 \mathrm{~s}$ and then allowed to stand for $15 \mathrm{~min}$ on ice. Fractions were then centrifuged at $25,000 \times \mathrm{g}$ for $10 \mathrm{~min}^{152}$. Pellets were resuspended in $0.25 \mathrm{M}$ sucrose/0.02 $\mathrm{M}$ Tris buffer. The rat liver and intestinal microsomes were stored at $-80^{\circ} \mathrm{C}$. The total protein concentration was determined by BioRad $R C D C$ Protein Assay Kit using BSA as a standard.

\subsubsection{Enterolactone Glucuronide Synthesis}

EL glucuronide (EL-Glu) was enzymatically synthesized by incubating with pooled human and rat liver and intestinal microsomes, respectively, at room temperature for $22 \mathrm{~h}$. The incubation experiment was conducted basically as described in a previous study ${ }^{124}$, while some experimental conditions were changed. Briefly, the incubation mixture $(1 \mathrm{~mL})$ consisted of $5 \mathrm{mM}$ uridine 5'-diphosphoglucuronic acid trisodium salt, $5 \mathrm{mM} \mathrm{MgCl}_{2}, 5 \mathrm{mM}$ D-saccharic acid 1,4-lactone monohydrate, $100 \mathrm{mM}$ Trizma base (adjusted with $\mathrm{HCl}$ to $\mathrm{pH} 7.4$ ), $2 \mathrm{mM} \mathrm{EL}$ 
and microsomal protein $(3.1 \mathrm{mg} / \mathrm{mL})$. The reaction was initiated by adding uridine 5'-diphosphoglucuronic acid trisodium salt. At the end of the incubation methanol (2 volumes) was added to terminate the reaction and precipitate proteins. After centrifugation of the mixture for $10 \mathrm{~min}$ at $10,000 \times \mathrm{g}$ in an Eppendorf microcentrifuge (Model 5417C, Brinkmann Instruments, Westbury, NY), the supernatant was concentrated by rotary evaporation and made up to its original volume.

\subsubsection{Purification of EL Glucuronide Conjugates}

The mixture of EL-Glu generated from rat liver microsomes was purified by HPLC Waters 600 system. The HPLC system consisted of Waters Model 600 solvent delivery system, Model 2996 photodiode Array Detector with the fixed wavelength at $280 \mathrm{~nm}$, Model 717 plus autosampler. All chromatographic separations were carried out on a reversed-phase semi-preparative column (Allsphere ODS-2 300×10 mm I.D., $5 \mu \mathrm{m}$ particle size). The analytes were eluted under gradient mode with mobile phase consisting of water with $0.1 \%$ formic acid (component $\mathrm{A}$ ) and acetonitrile with $0.1 \%$ formic acid (component B) in different ratios delivered at a flow rate of $3 \mathrm{~mL} / \mathrm{min}$. The gradient consisted of 85:15 component $\mathrm{A}$ : component B from 0 to $12 \mathrm{~min}$, an increasing gradient from $15 \%$ to $50 \%$ component $\mathrm{B}$ and from $50 \%$ to $90 \%$ between 12 and $14 \mathrm{~min}$ and then $90 \%$ between 14 and $18 \mathrm{~min}$, with a decreasing gradient from $90 \%$ to $15 \%$ component B from 18 to $20 \mathrm{~min}$ and a return to $85: 15$ component A: component B between 20 and 25 min. Fractions containing glucuronide at the desired retention time were collected automatically by Waters Fraction Collector, and solvents were removed by rotary evaporation.

\subsubsection{Characterization of EL Glucuronide}

Liquid chromatography-mass spectrometry (LC-MS) was used to verify and confirm the formation and structure of the purified fractions containing EL glucuronide. The LC-MS/MS analysis was conducted on a Hybrid Triple Quadrupole/Linear Ion Trap mass spectrometer (AB Sciex 4000 QTRAP, MS/MS system, Applied Biosystems Inc., Foster City, CA) fitted with Turbo V electrospray ionization (ESI) source in the negative mode, coupled with Agilent 
Technologies 1100 Series LC which consisted of a binary pump and autosampler. The chromatographic separation was performed on a Waters Symmetry Reverse-Phase C18 column $(150 \times 4.6 \mathrm{~mm}$ I.D., $5 \mu \mathrm{m}$ particle size $)$. The gradient was the same as described above. The flow rate was delivered at $1 \mathrm{~mL} / \mathrm{min}$. The MS parameters were optimized for EL-Glu as follows: declustering potential (DP) $-90 \mathrm{~V}$, collision energy (CE) $-36 \mathrm{~V}$, Collision Cell Exit Potential (CXP) $-23 \mathrm{~V}$. The ion source parameters were curtain gas (CRU) $10 \mathrm{~V}$; ion spray voltage (IS) -4500 V; temperature (TEM) 400; ion source gas 1 (GS1), 40 psi; ion source gas 2 (GS2), 40 psi; collision activated dissociation (CAD) medium; entrance potential (EP) -10 V. The fragmentation transitions for multiple reaction monitoring (MRM) were mass to charge ratio $(m / z) 473.0 \rightarrow 297.0$ and $473.0 \rightarrow 175.0$.

\subsection{Experiment Two: HPLC Method}

A reliable and simple HPLC method for the quantitative determination of flaxseed lignans was established in our lab ${ }^{102}$. Hence, a partial HPLC method validation was conducted to allow for the quantitative determination of EL in this study.

\subsubsection{Instrumentation and Chromatographic Conditions}

The HPLC (Agilent Technologies, Mississauga, ON) system consisted of a Series 1200 quaternary pump (G1311A) with online degasser (G1322A), autosampler (G1329A) and fluorescence detector (G1321A). Excitation wavelength was set at $277 \mathrm{~nm}$ and emission wavelength at $617 \mathrm{~nm}$. Processed samples $(50 \mu \mathrm{L})$ were injected onto a Waters Symmetry Reverse-Phase C18 column (150×4.6 mm I.D., $5 \mu \mathrm{m}$ particle size). The analytes were eluted under gradient mode with mobile phase consisting of water with $0.1 \%$ formic acid (component A) and acetonitrile with $0.1 \%$ formic acid (component B) in different ratios delivered at a flow rate of $1 \mathrm{~mL} / \mathrm{min}$. The gradient consisted of 85:15 component A: component B from 0 to 12 min, an increasing gradient from $15 \%$ to $50 \%$ component B and from $50 \%$ to $90 \%$ between 12 and $14 \mathrm{~min}$ and then $90 \%$ between 14 and $18 \mathrm{~min}$, with a decreasing gradient from $90 \%$ to $15 \%$ component $\mathrm{B}$ from 18 to $20 \mathrm{~min}$ and a return to $85: 15$ component $\mathrm{A}$ : component $\mathrm{B}$ between 20 and $25 \mathrm{~min}$. 


\subsubsection{Validation of HPLC Method}

HPLC method validation procedures were performed based on FDA guidelines. Specificity was assessed by analyzing pooled human liver microsomes (HLM), human intestinal microsomes (HIM), rat duodenum microsomes (RDM), rat jejunum microsomes (RJM), rat colon microsomes (RCM) and six different rat liver microsomes (RLM), whose preparations were supplemented only with internal standard (umbelliferone) to determine the absence of endogenous substances with similar retention time as EL and/or EL-Glu.

The sensitivity of the method was evaluated by determination of the limit detection (LOD) and the lowest limit of quantification (LLOQ). LOD was defined as the lowest detectable concentration, considering the signal-to-noise ratio of 3. The lowest limit of quantification (LLOQ) was the lowest concentration of the standard curve that gave acceptable accuracy and precision. Accuracy (\%) was expressed as [(calculated amount/predicted amount)*100] with acceptable limits at $15 \%$ except at LLOQ, where it should not deviate by more than $20 \%$. Precision was expressed as \% relative standard deviation (RSD) with acceptable level at 15\% of the coefficient of variation (CV) except at LLOQ, where it should not exceed $20 \%$ of CV. The intra-day accuracy and precision were evaluated by analyzing six replicates of quality control (QC) samples (low, medium and high concentrations of quality controls) on a single day. The inter-day accuracy and precision were determined from the same QC samples on three different days.

The linearity of the method was assessed by processing a 6-point calibration curve on several different days. The ratios of the peak areas of analytes and the internal standard were plotted against the nominal concentrations of the analytes. A linear least-squares regression analysis using $1 / X^{2}$ as weighting factor was conducted to determine slope, intercept and coefficient of determination $\left(r^{2}\right)$ to demonstrate linearity of the method.

\subsection{Experiment Three: Enzyme Kinetic Analysis}

Enzyme kinetics were conducted using pooled HLM, HIM, RLM, RJM and RCM, respectively. Pooled human liver (pool of 50) and intestinal microsomes (pool of 13) from 
mixed gender were purchased from XenoTech, LLC (Lenexa, KS). Human intestinal microsomes were prepared from duodenal and jejunal tissues. Pooled rat liver (pool of 3) and jejunum and colon (pool of 2) from male gender were extracted based on established protocols in our lab. Comparison of EL glucuronidation between species and tissues was assessed by determination of intrinsic clearance $\left(\mathrm{CL}_{\text {int }}\right)$ in intestinal and liver microsomes. $\mathrm{CL}_{\text {int }}$ (as a measure of the maximal efficacy of enzyme to metabolize substrate) was determined by the substrate depletion method, in which the depletion of substrate was monitored as a function of time $e^{153}$.

The incubation mixture consisted of (at their final concentrations): microsomes $(0.5$ $\mathrm{mg} / \mathrm{mL}$ ), $5 \mathrm{mM} \mathrm{MgCl}_{2}, 5 \mathrm{mM}$ D-saccharic acid 1,4-lactone monohydrate, $100 \mathrm{mM}$ Trizma base (adjusted with $\mathrm{HCl}$ to $\mathrm{pH}$ 7.4). A range of final EL concentrations (10-500 $\mu \mathrm{M})$ was incubated with HLM and RLM, respectively, while EL concentrations (10-300 $\mu \mathrm{M})$ were incubated with HIM. The total incubation volume for all of three incubations was $1.5 \mathrm{~mL}$. EL concentrations $(1.5-100 \mu \mathrm{M})$ were used when incubating with RJM and RCM with a total volume of $1.0 \mathrm{~mL}$. Trizma base ( $\mathrm{pH} \mathrm{7.4)} \mathrm{containing} \mathrm{uridine} \mathrm{5'-diphosphoglucuronic} \mathrm{acid}$ trisodium salt $\left(5 \mathrm{mM}\right.$ final concentration) preincubated at $37^{\circ} \mathrm{C}$ for $5 \mathrm{~min}$ in a shaking water bath was used to initiate the reaction. At multiple incubation time points, aliquots $(0.2 \mathrm{~mL})$ in the incubations containing HLM, HIM and RLM were withdrawn and $20 \mu \mathrm{L}$ internal standard (1.23 $\mathrm{mM}$ umbelliferone) in acetonitrile was added to the aliquots. At equivalent incubation time points, aliquots $(130 \mu \mathrm{L})$ in the incubations containing RJM and RCM were withdrawn and $13 \mu \mathrm{L}$ of internal standard in acetonitrile was added. Subsequently, the aliquots were immersed into the liquid nitrogen immediately to terminate the reaction. After thawing on the ice, the mixture was centrifuged at $10,000 \times \mathrm{g}$ for $10 \mathrm{~min}$ in an Eppendorf microcentrifuge (Model 5417C, Brinkmann Instruments, Westbury, NY). $50 \mu \mathrm{L}$ of the supernatant (triplicate) for HLM, HIM and RLM incubations as well as duplicates $(50 \mu \mathrm{L})$ for RJM and RCM incubations was injected on the Waters Symmetry Reverse-Phase C18 column $(150 \times 4.6 \mathrm{~mm}$ I.D., $5 \mu \mathrm{m}$ particle size) for the immediate HPLC analysis of EL. Heated microsomes, no EL and no uridine 5'-diphosphoglucuronic acid trisodium salt incubation reactions were used as negative controls. 


\subsection{Data Analysis}

To determine $\mathrm{CL}_{\text {int, }}$, kinetic parameters in microsomal preparations were estimated with the methods reported by both Obach and Reed-Hangen and Komura and Iwaki 154,155. Analyte/internal standard peak area were determined and normalized to the ratio obtained at time, $\mathrm{t}=0$, which represents $100 \%$. A plot of natural log EL percent remaining versus time was constructed to calculate the substrate depletion rate constant ( $\left.k_{\mathrm{dep}}\right)$ (the slope of each linear line) for each EL concentration. If substrate decline demonstrated nonlinearity on log percentage remaining versus time curves, only those initial time points wherein log linearity was observed were used to determine depletion rate constants. The $K_{\mathrm{m}}$ and $k_{\operatorname{dep}(\mathrm{S}] \rightarrow 0)}$ values were determined by plotting depletion rate constants $\left(k_{\mathrm{dep}}\right)$ versus various initial EL concentrations by a nonlinear least squares regression analysis with Prism 4.0 software (GraphPad Prism, San Diego, CA, USA) using the following equation:

$$
k_{\text {dep }}=k_{\operatorname{dep}([S] \rightarrow 0)} \cdot\left(1-\frac{[S]}{[S]+K_{m}}\right)
$$

where $k_{\text {dep }}$ is the apparent first-order rate constant of substrate depletion, $[S]$ is the substrate concentration and $k_{\operatorname{dep}[[\mathrm{S}] \rightarrow 0)}$ is the theoretical maximum consumption rate constant at an infinitesimally low substrate concentration and $K_{\mathrm{m}}$ is the Michaelis-Menten constant. The intrinsic clearance at an infinitesimally low substrate concentration $\left(\mathrm{CL}_{\text {int, app }(\mathrm{S}]=0)}\right)$ was calculated by dividing $k_{\mathrm{dep}([\mathrm{S}] \rightarrow 0)}$ by the microsomal protein concentration used. $\mathrm{V}_{\max }$ was derived from generated $K_{\mathrm{m}}$ and $\mathrm{CL}_{\text {int, app }(\mathrm{S}]=0)}$. 


\section{RESULTS}

\subsection{EL Glucuronide Synthesis with Rat and Human Microsomes}

EL glucuronide was enzymatically generated by incubation with rat liver and intestinal (duodenum, jejunum and colon) microsomes, human liver and intestinal microsomes, respectively, at room temperature for $22 \mathrm{~h}$ as most of the EL was converted to EL glucuronide in this time frame. HPLC (Waters 600 system, semi-preparative column) analysis of EL glucuronide from rat liver microsomes (RLM) resulted in the appearance of a single product peak. The retention time for EL glucuronide (EL-Glu) was $14.1 \mathrm{~min}$, and some EL was unreacted and observed in the incubation mixtures (Figure 4.1A). After purification of EL-Glu from RLM by semi-preparative HPLC, three milligrams of EL-Glu was collected and no EL was detected in this sample (Figure 4.1B). However, I found that the peak shape of purified EL-Glu was not symmetrical, indicating the presence of more than one form of EL-Glu. Accordingly, LC-MS/MS was used to identify and confirm the formation and structures of the purified fractions containing EL-Glu. MS analysis suggested that the glucuronide for EL from RLM predominantly occurred as the monoglucuronide with an $m / z$ of 473 . The optimized fragments for EL monoglucuronide were 297 and 175, which corresponded with the cleavage of the bond between EL and glucuronic acid (Figure 4.2).

Multiple Reaction Monitoring (MRM) was also employed to further oversee the formation of EL monoglucuronide from each tissue and species. According to two peaks observed with very close retention times around $9 \mathrm{~min}$, the data suggested the formation of two monoglucuronides for EL incubated with RLM (Figure 4.3). Interestingly, only one peak was observed by MRM for EL-Glu from rat intestinal microsomes (Figure 4.4A-C), human liver and intestinal microsomes (Figure 4.4D-E). In addition, preliminary microsomal incubation results showed that colon made a greater contribution to EL glucuronidation than jejunum, but both tissues were dramatically greater than that of duodenum in rat intestine. Hence, only colon and jejunum were used to further explore the enzyme kinetics for EL glucuronidation in rat intestine. Human liver produced EL-Glu more efficiently than human intestine. 


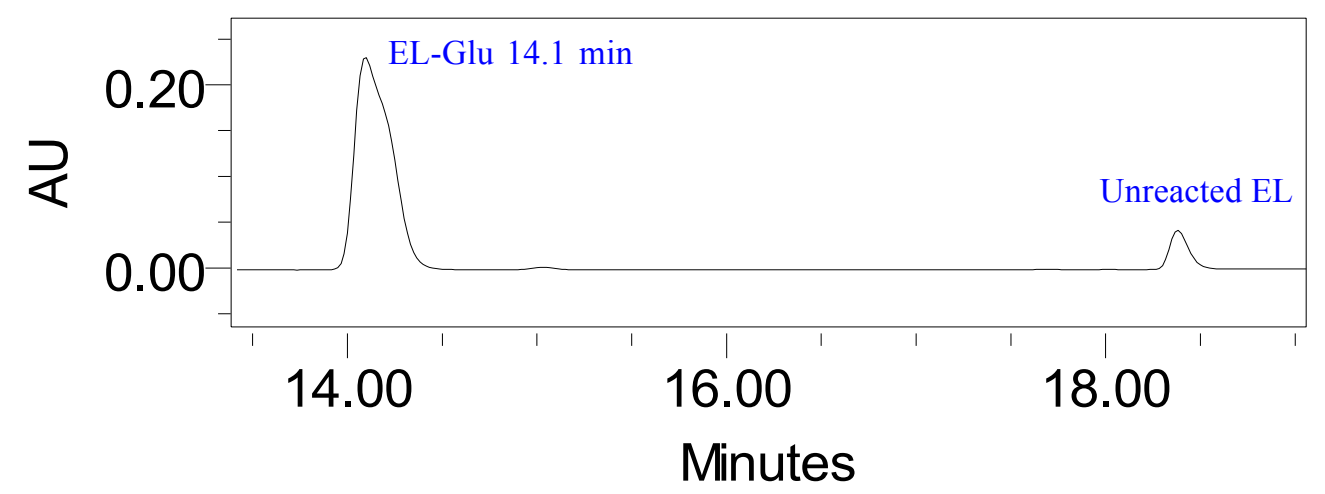

A

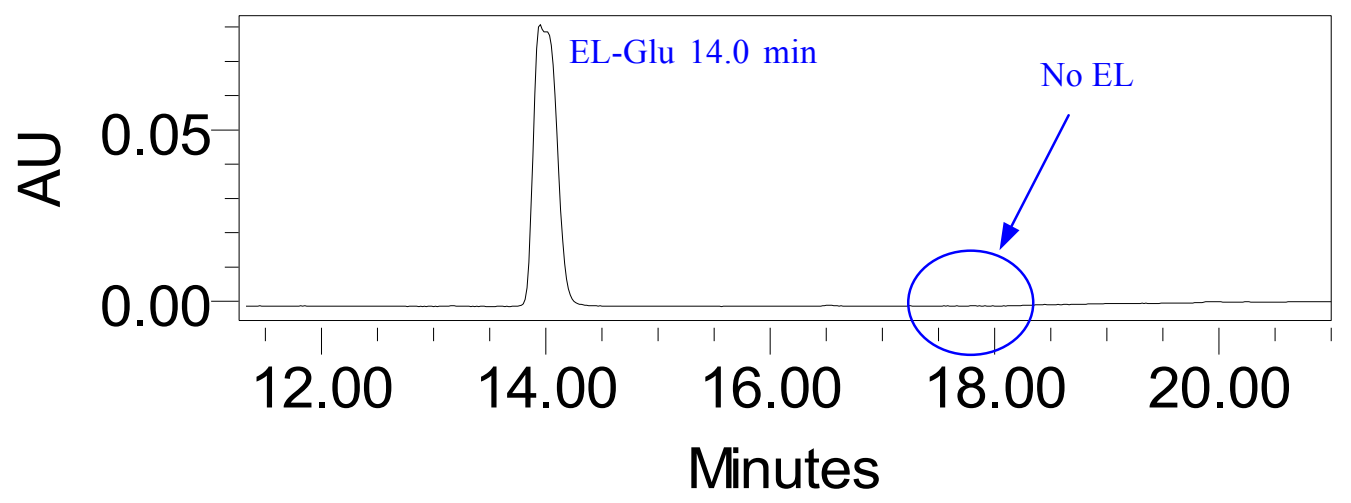

B

Figure 4.1 Representative HPLC chromatograms of unpurified EL glucuronide (A) and purified EL glucuronide (B) generated from rat liver microsomes. Conditions: Waters 600 HPLC system; reversed-phase semi-preparative column $(300 \times 10 \mathrm{~mm}$ I.D., $5 \mu \mathrm{m}$ particle size); component A: water with $0.1 \%$ formic acid, component $\mathrm{B}$ : acetonitrile with $0.1 \%$ formic acid; detection wavelength at $280 \mathrm{~nm}$. 


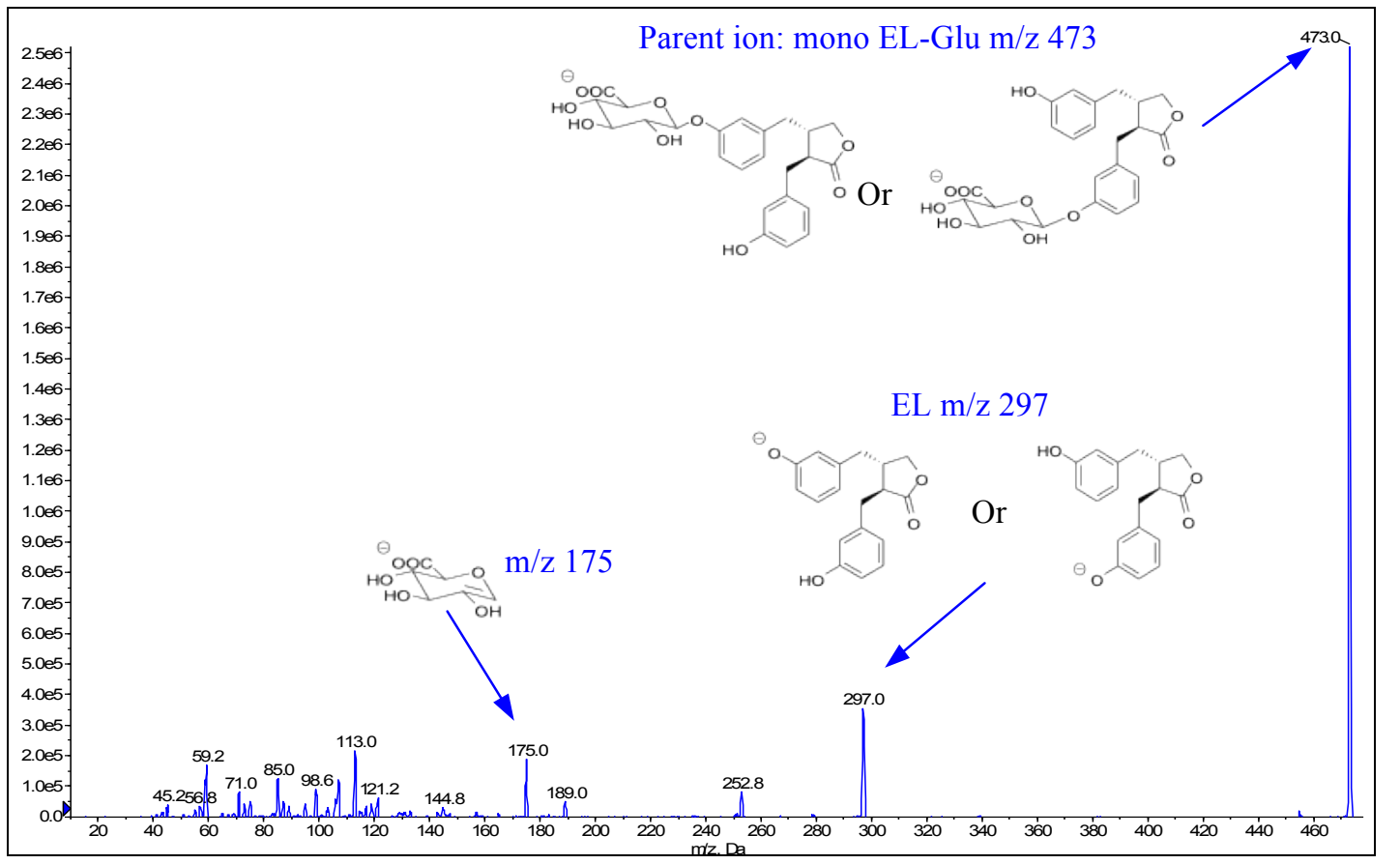

Figure 4.2 LC-MS analysis of proposed EL glucuronide $(\mathrm{m} / \mathrm{z} 473)$ formed from rat liver microsomes obtained in negative electrospray ionization (ESI) mode 


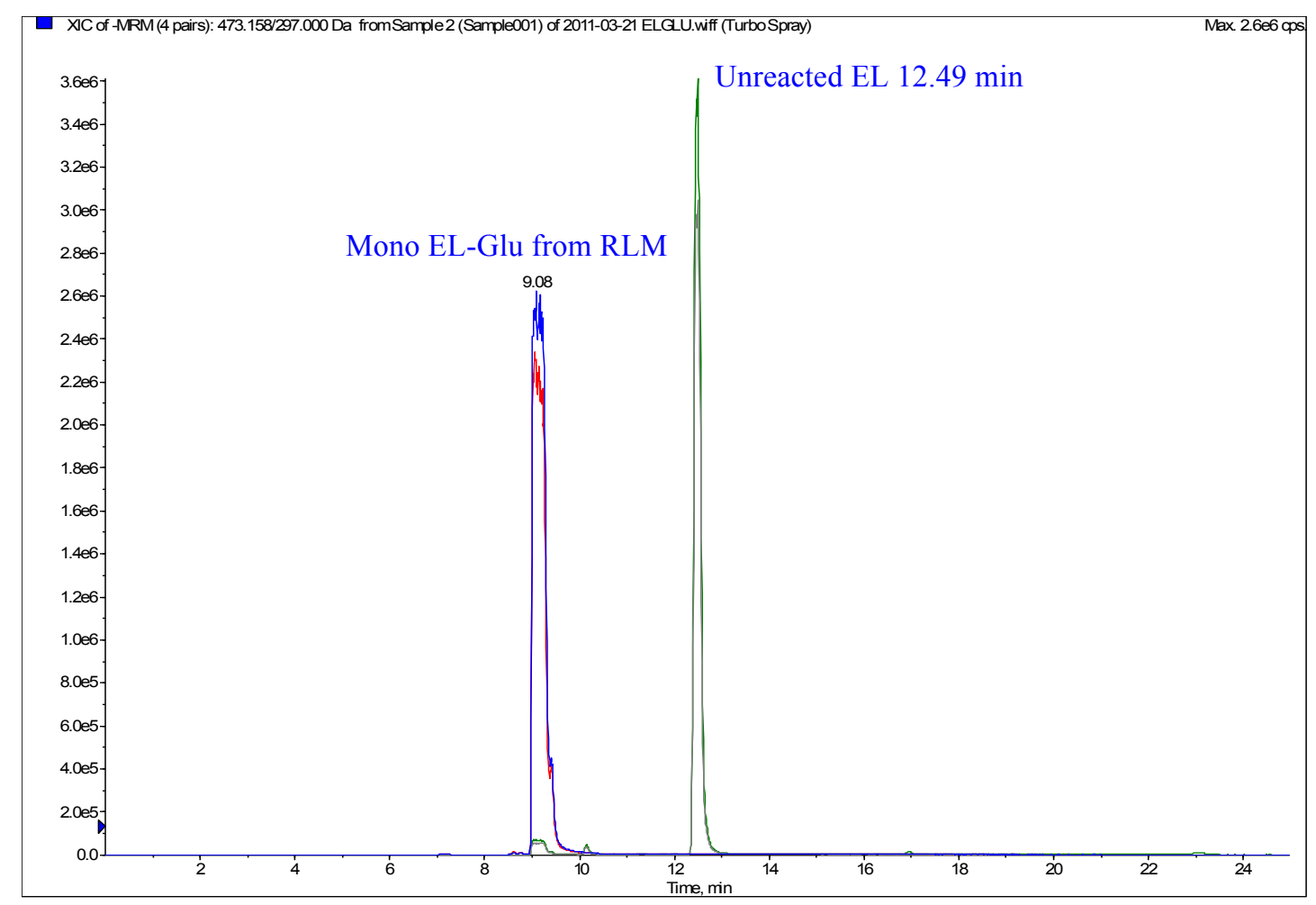

A

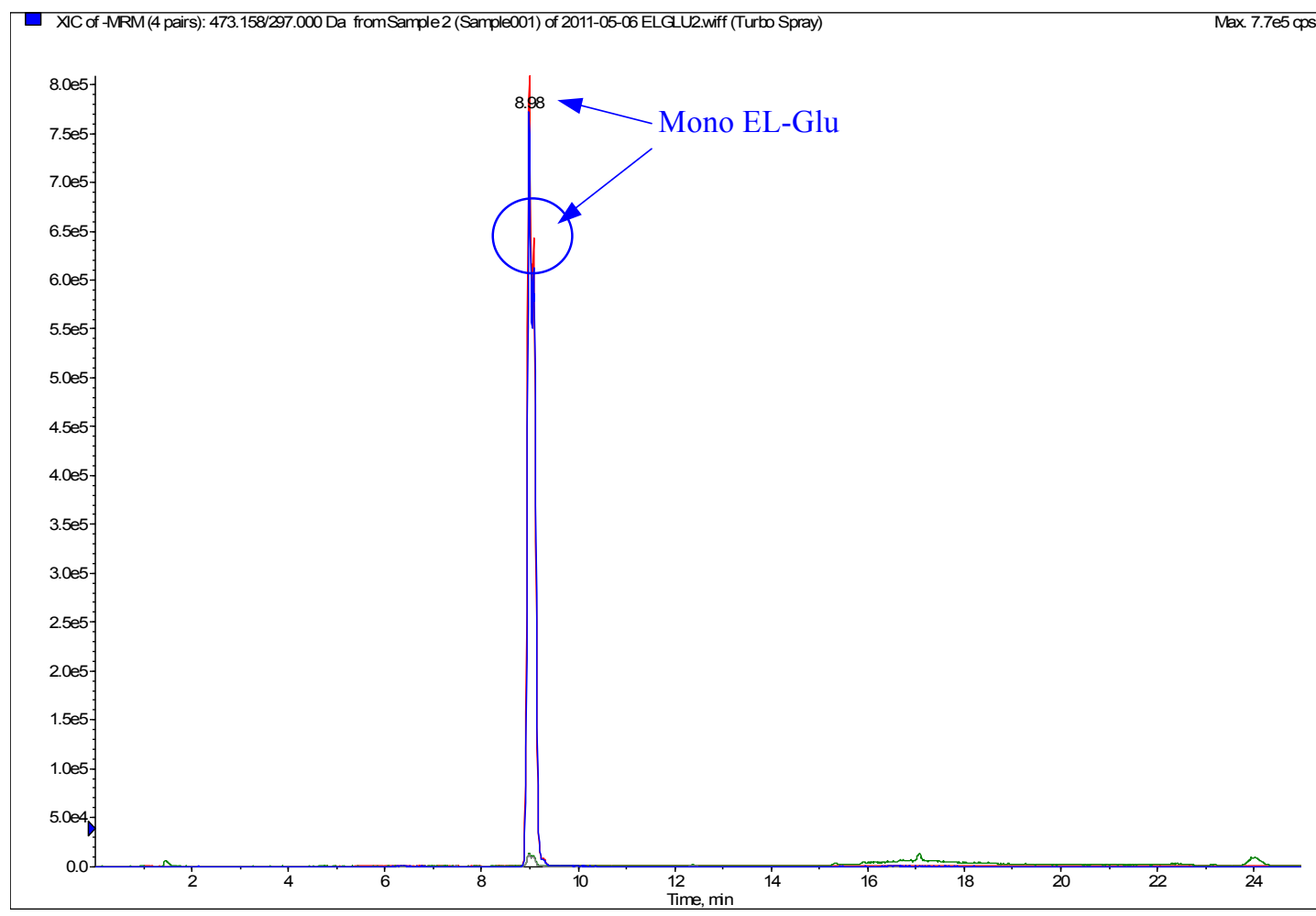

B

Figure 4.3 Multiple reaction monitoring chromatograms of unpurified EL glucuronide (A) and purified EL glucuronide (B) generated from rat liver microsomes (RLM) 


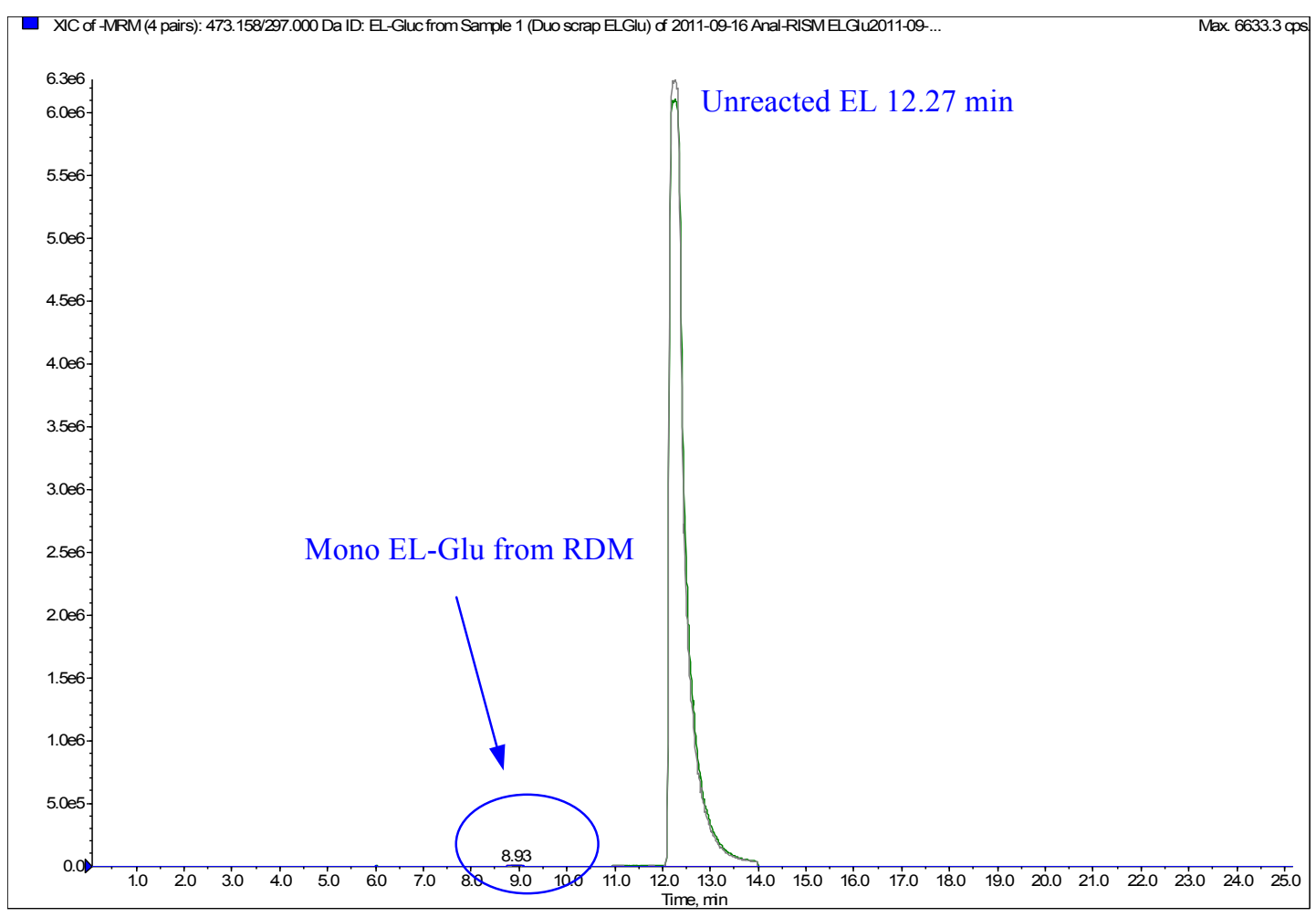

A

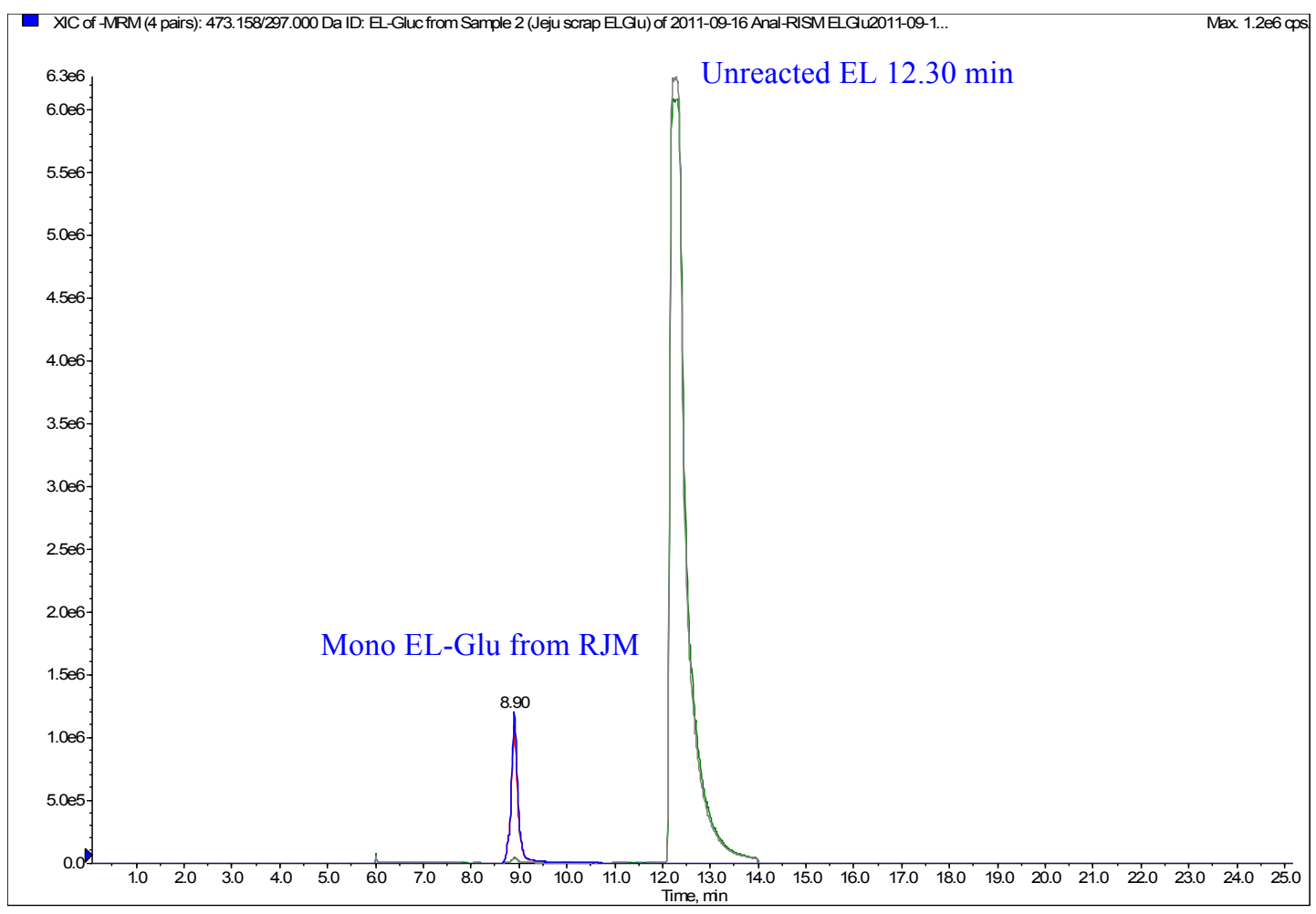




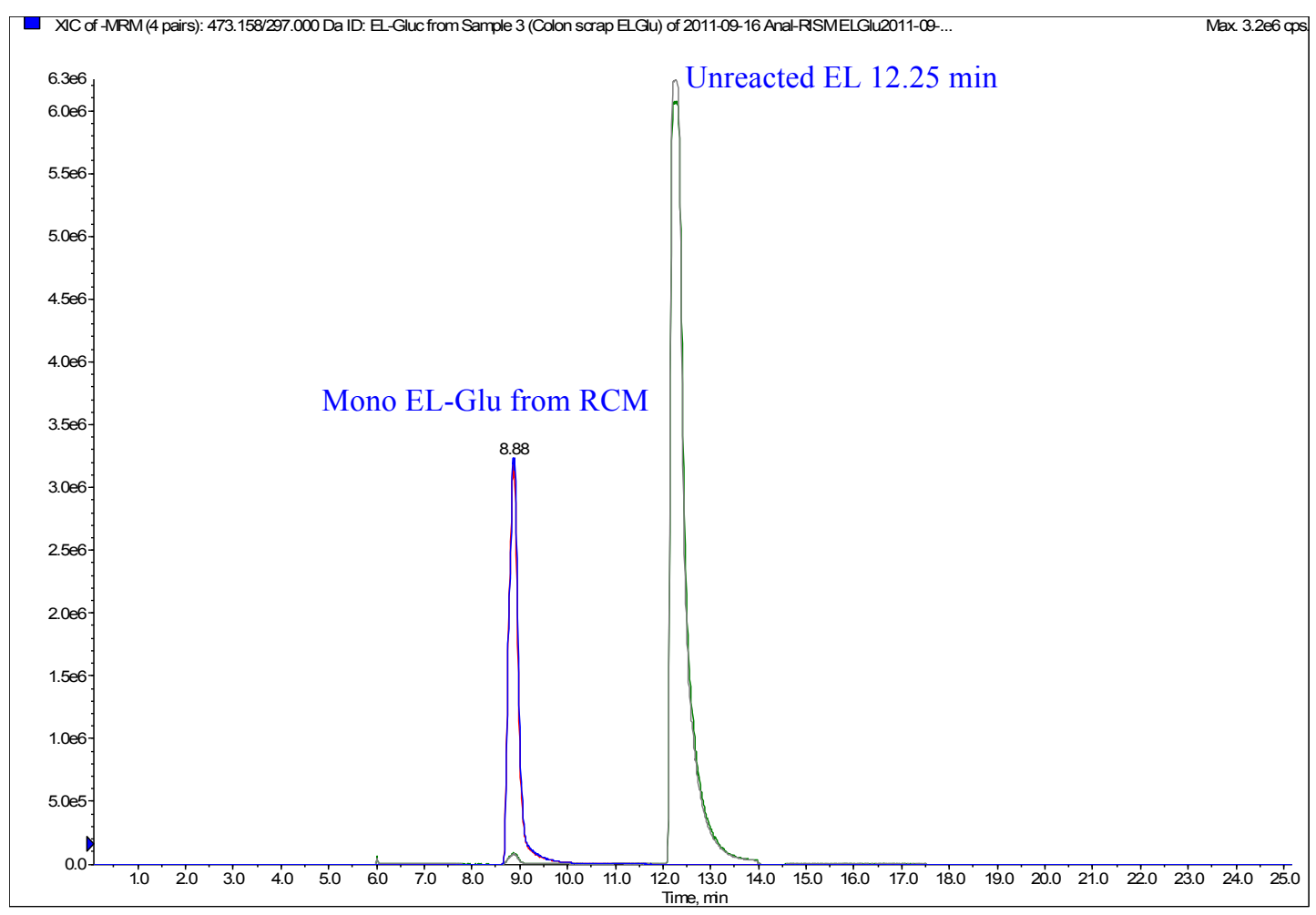

C

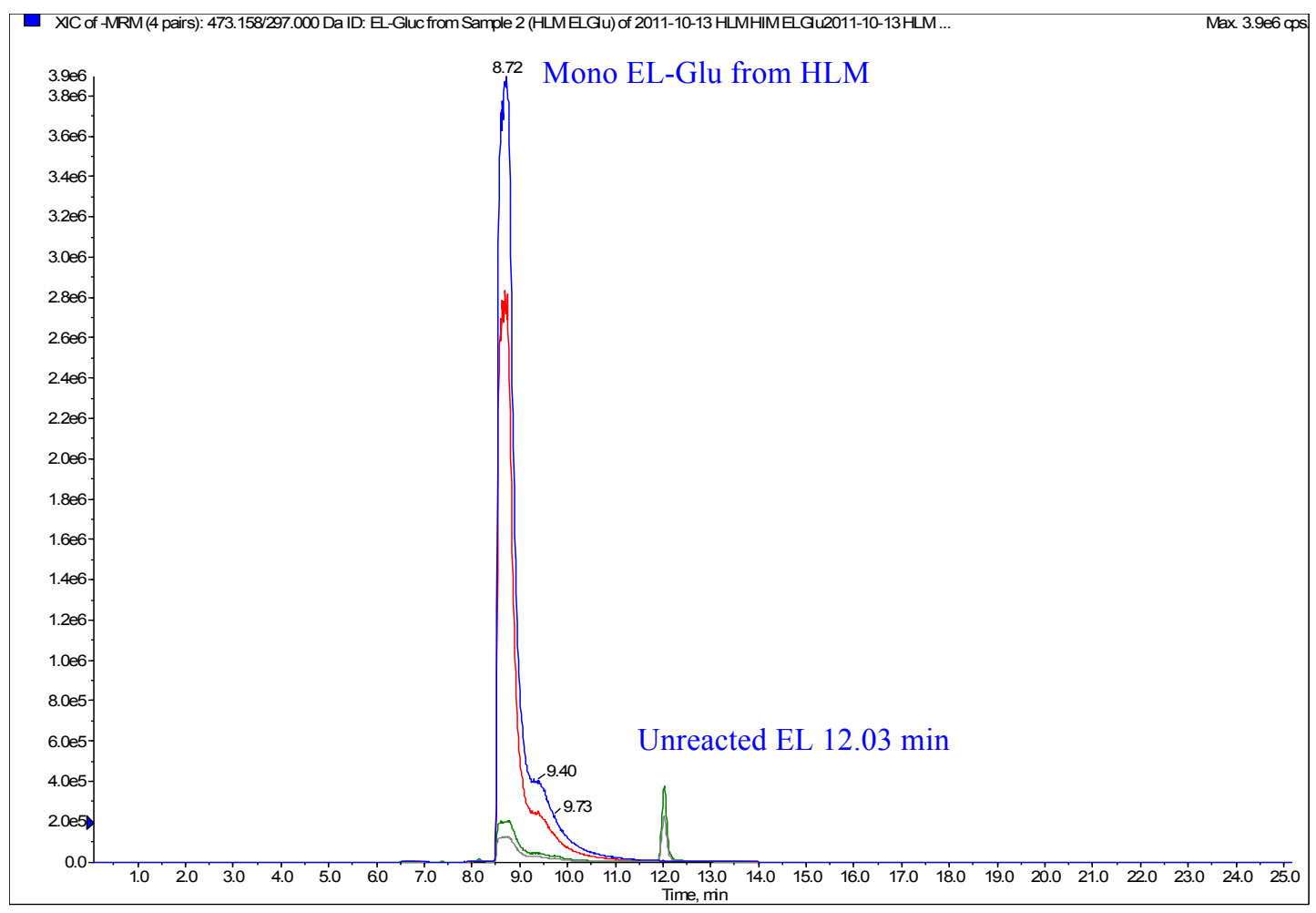

D 


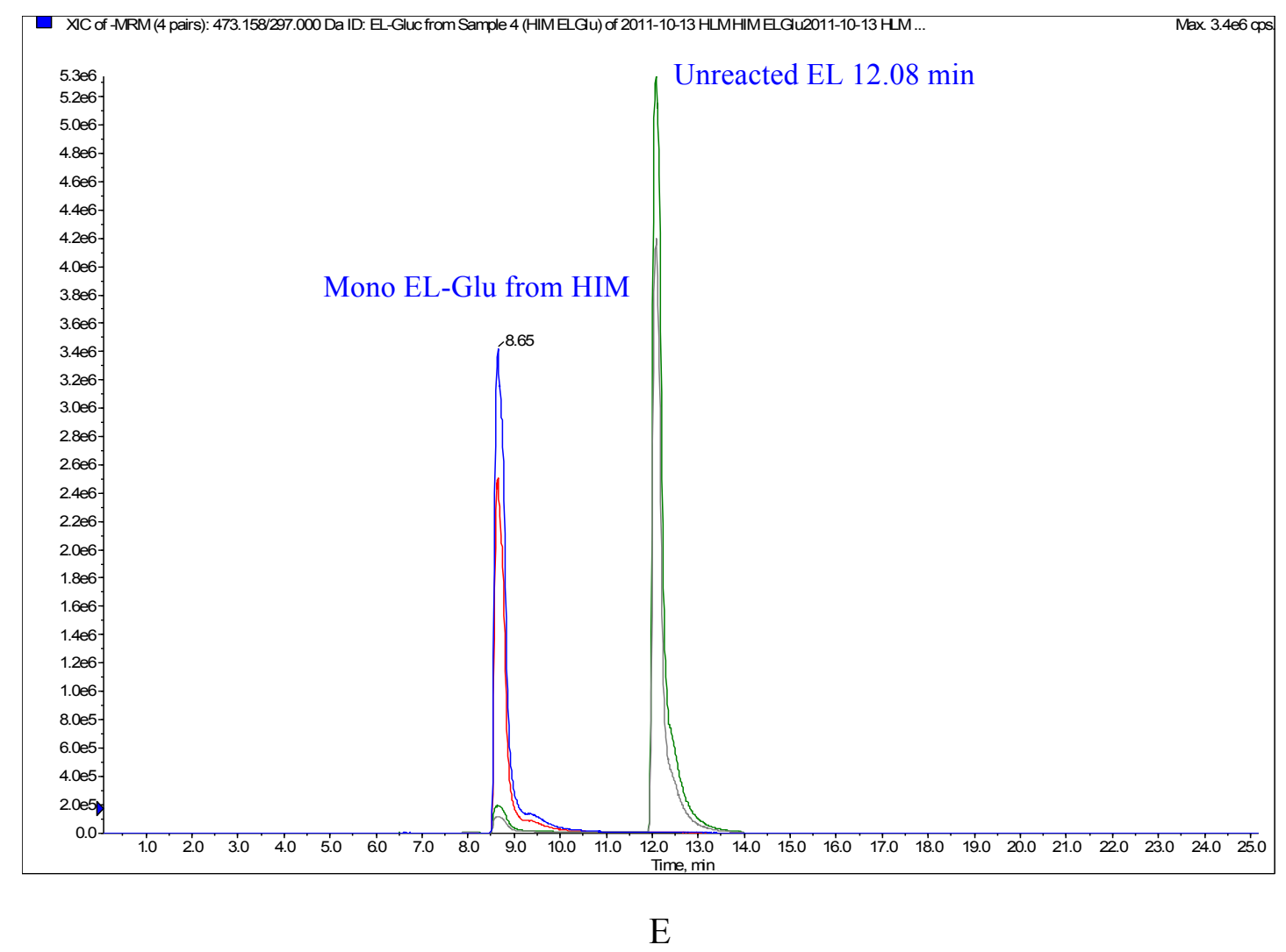

Figure 4.4 Multiple reaction monitoring analysis of EL glucuronide incubated with rat duodenum microsomes (RDM) (A), rat jejunum microsomes (RJM) (B), rat colon microsomes $(\mathrm{RCM})(\mathrm{C})$, human liver microsomes (HLM) (D) and human intestinal microsomes (HIM) (E) 


\subsection{HPLC Method Validation for EL}

A HPLC method was validated for the quantitative determination of EL in RLM. Figure 4.5 represented HPLC chromatograms for blank rat liver microsomes and EL, and its internal standard (umbelliferone) in RLM. No endogenous substances were found in RLM, RCM, HLM and HIM that interfered with EL. The chromatographic conditions gave retention times for internal standard (umbelliferone) and EL as 6.8 and $12.0 \mathrm{~min}$, respectively. The recovery of EL was 104.9, 110.6 and 109.8\% at LQC, MQC and HQC, respectively. The lower limit of quantification (LLOQ) for EL was $41.7 \mathrm{ng} / \mathrm{mL}$. Table 4.1 and Table 4.2 summarize the intraand interday precision and accuracy data. Overall, the intra- and interday precision and accuracy for EL of this HPLC method were $\leq 10 \%$, suggesting the method was both precise and accurate to quantitatively determine EL in rat and human liver and intestinal microsomes. 


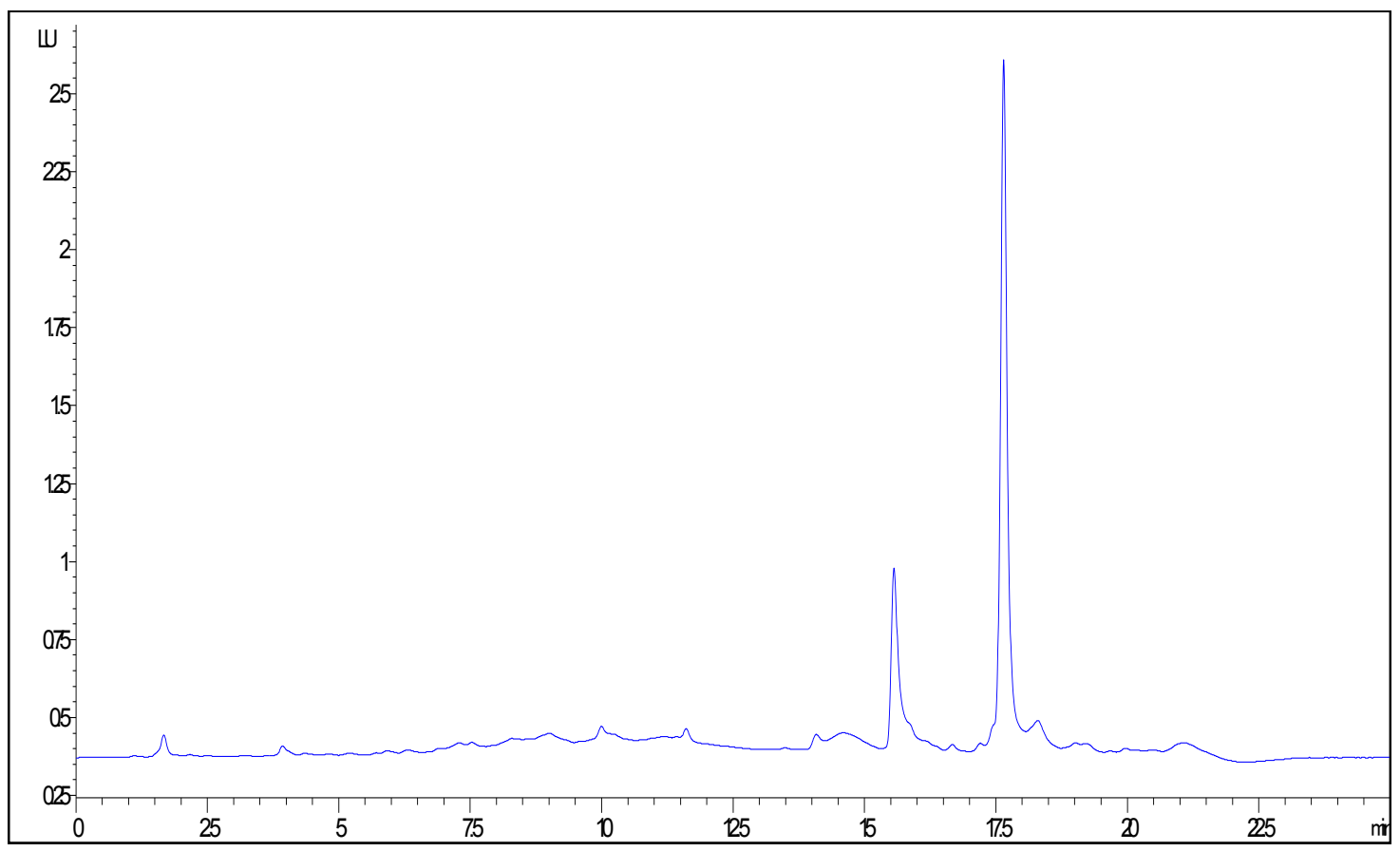

A

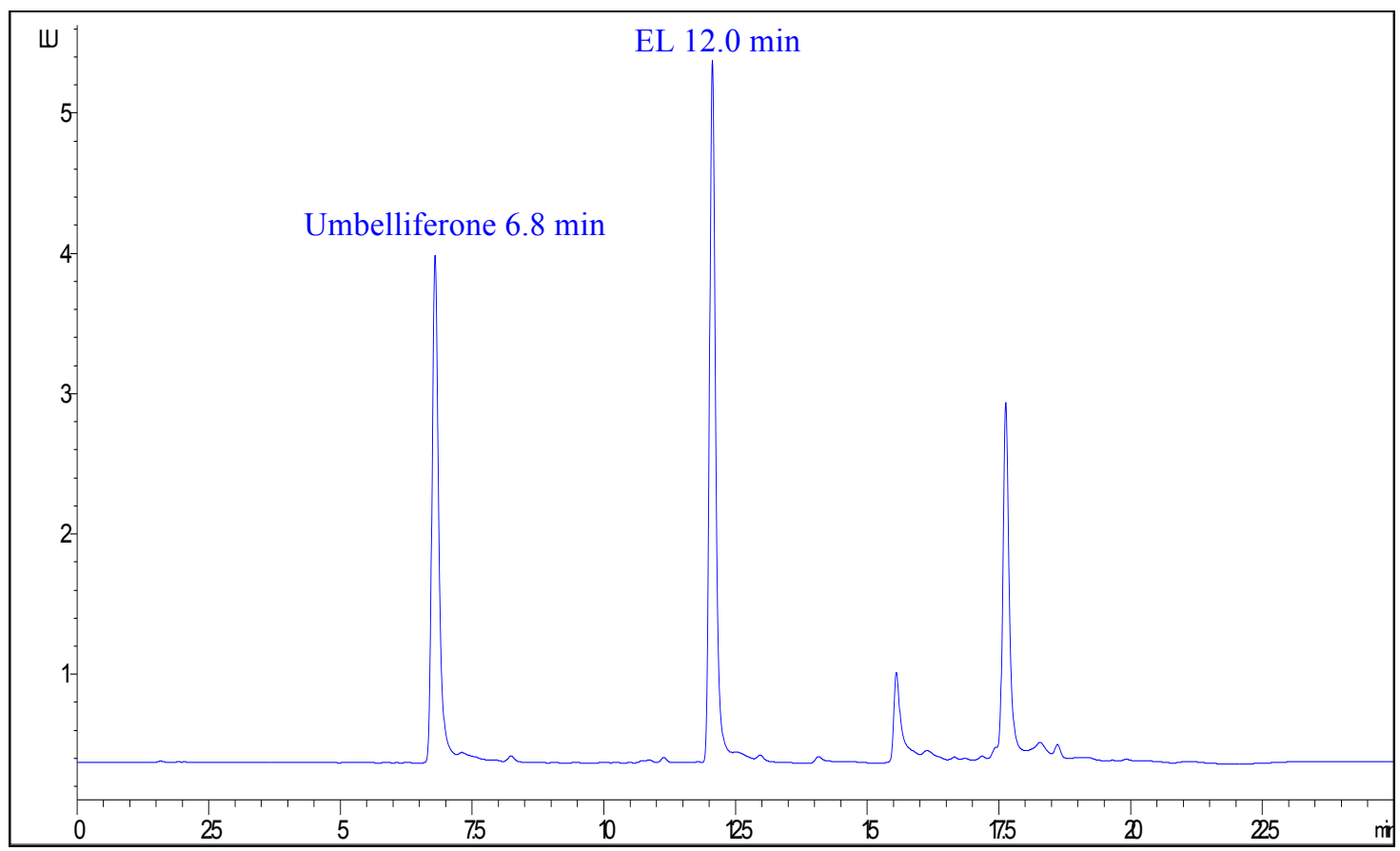

$\mathrm{B}$

Figure 4.5 Representative HPLC chromatograms of blank rat liver microsomes (A) and rat liver microsomes spiked with internal standard umbelliferone $(100 \mu \mathrm{g} / \mathrm{mL}, 6.8 \mathrm{~min})$ and EL (4 $\mu \mathrm{g} / \mathrm{mL}, 12.0 \mathrm{~min}$ ) (B). Conditions: Agilent 1200 HPLC system; Waters Symmetry reversed-phase $\mathrm{C} 18$ analytical column $(150 \times 4.6 \mathrm{~mm}$ I.D., $5 \mu \mathrm{m}$ particle size); component $\mathrm{A}$ : water with $0.1 \%$ formic acid, component $\mathrm{B}$ : acetonitrile with $0.1 \%$ formic acid; excitation wavelength at $277 \mathrm{~nm}$ and emission wavelength at $617 \mathrm{~nm}$. 
Table 4.1 Intraday assay precision and accuracy for EL in rat liver microsomes $(\mathrm{N}=6)$

\begin{tabular}{lcc} 
QC levels & Precision & Accuracy \\
LLOQ & & \\
Day 1 & 2.7 & 92.7 \\
Day 2 & 1.6 & 101.4 \\
Day 3 & 4.8 & 101.1 \\
LQC & & \\
Day 1 & 4.4 & 90.0 \\
Day 2 & 4.3 & 100.6 \\
Day 3 & 2.8 & 95.4 \\
MQC & & \\
Day 1 & 3.3 & 94.7 \\
Day 2 & 2.2 & 106.5 \\
Day 3 & 4.0 & 100.2 \\
HQC & & \\
Day 1 & 1.5 & 93.9 \\
Day 2 & 2.8 & 102.3 \\
Day 3 & 3.1 & 97.0 \\
\hline
\end{tabular}

* Precision is expressed as $\mathrm{CV} \%=\frac{\text { standard deviation }}{\text { mean }} \times 100 \%$

* Accuracy is calculated as $\frac{\text { mean determined concentration }}{\text { actual concentration }} \times 100 \%$

* LLOQ for EL is $41.7 \mathrm{ng} / \mathrm{mL}$; LQC for EL is $150 \mathrm{ng} / \mathrm{mL}$; MQC is $4000 \mathrm{ng} / \mathrm{mL}$ and HQC is $8000 \mathrm{ng} / \mathrm{mL}$

Table 4.2 Interday assay precision and accuracy for EL in rat liver microsomes $(\mathrm{N}=6)$

\begin{tabular}{ccc} 
QC levels & Precision & Accuracy \\
LLOQ & 3.9 & 98.4 \\
LQC & 5.0 & 95.4 \\
MQC & 4.9 & 100.5 \\
HQC & 3.4 & 97.8 \\
\hline
\end{tabular}

* Precision is expressed as CV\% $=\frac{\text { standard deviation }}{\text { mean }} \times 100 \%$

* Accuracy is calculated as $\frac{\text { mean determined concentration }}{\text { actual concentration }} \times 100 \%$

* LLOQ for EL is $41.7 \mathrm{ng} / \mathrm{mL}$; LQC for EL is $150 \mathrm{ng} / \mathrm{mL}$; MQC is $4000 \mathrm{ng} / \mathrm{mL}$ and HQC is $8000 \mathrm{ng} / \mathrm{mL}$ 


\subsection{In Vitro Hepatic and Intestinal Microsomal Intrinsic Clearance of EL}

The in vitro $\mathrm{CL}_{\mathrm{int}}$ values of EL glucuronidation were examined using RLM, RJM and RCM extracted in our lab and commercial sources of HLM and HIM (XenoTech, LLC) by the substrate depletion approach $^{154}$ (Table 4.3). Control assays conducted with heated microsomes and without microsomes and uridine 5'-diphosphoglucuronic acid trisodium salt exhibited no substrate depletion over time. The relationships between the first-order constants for substrate depletion (termed $k_{\text {dep }}$ ) and EL concentrations for EL glucuronidation were shown in Figure 4.6. Typical examples of the process to generate $k_{\text {dep }}$ value were exhibited in Appendix I. $k_{\text {dep }}$ values for each substrate concentration for rat and human liver and intestinal microsomes were summarized in Appendix I. The in vitro $\mathrm{CL}_{\text {int }}$ values for EL glucuronidation determined with RLM and HLM were much greater than those for intestinal microsomes, and the HLM (1.18 $\mathrm{mL} / \mathrm{min} / \mathrm{mg}$ ) gave the highest value. RJM and $\mathrm{RCM}$ gave comparable $\mathrm{CL}_{\text {int }}$ values; on the other hand, they were considerably lower than HIM (Figure 4.7A). Similar trends also applied to $\mathrm{V}_{\max }$ values, nonetheless, RLM showed the highest $\mathrm{V}_{\max }$ value (Figure 4.7B). The $K_{\mathrm{m}}$ values for EL glucuronidation evaluated using rat microsomes were significantly larger than human microsomes among which RCM had the highest $K_{\mathrm{m}}$ value as $45.9 \mu \mathrm{M}$. Rat intestinal microsomes generated greater $K_{\mathrm{m}}$ values than rat liver microsomes, the results of which were in agreement with that of human (Figure 4.7C). 
Table 4.3 $\mathrm{CL}_{\text {int }}$ and Michaelis-Menten constants of EL glucuronidation by rat and human liver and intestinal microsomes

\begin{tabular}{cccccc}
\hline Microsomes & $\begin{array}{c}\mathbf{C L}_{\mathbf{i n t}} \\
\mathbf{m L} / \mathbf{m i n} / \mathbf{m g}\end{array}$ & $\begin{array}{c}\mathbf{9 5 \%} \mathbf{C I} \text { for } \\
\mathbf{C L}_{\mathbf{i n t}}\end{array}$ & $\begin{array}{c}\boldsymbol{K}_{\mathbf{m}} \\
\mathbf{\mu M}\end{array}$ & $\begin{array}{c}\mathbf{9 5 \%} \mathbf{C I} \\
\text { for } \boldsymbol{K}_{\mathbf{m}}\end{array}$ & $\begin{array}{c}\mathbf{V}_{\mathbf{m a x}} \\
\mathbf{n m o l} / \mathbf{m i n} / \mathbf{m g}\end{array}$ \\
\hline RLM & $1.00 \pm 0.1$ & $0.77-1.24$ & $25.6 \pm 5.07$ & $13.2-38.0$ & 25.7 \\
RJM & $0.05 \pm 0.003$ & $0.042-0.058$ & $31.6 \pm 7.72$ & $12.7-50.4$ & 1.58 \\
RCM & $0.058 \pm 0.003$ & $0.050-0.066$ & $45.9 \pm 11.4$ & $16.6-75.1$ & 2.65 \\
HLM & $1.18 \pm 0.09$ & $0.97-1.40$ & $8.91 \pm 1.07$ & $6.44-11.4$ & 10.6 \\
HIM & $0.31 \pm 0.027$ & $0.25-0.37$ & $12.6 \pm 1.84$ & $8.36-16.9$ & 3.90 \\
\hline
\end{tabular}

Different range of EL concentrations were incubated with liver and intestinal microsomes from human and rat $(0.5 \mathrm{mg} / \mathrm{mL})$ for $30 \mathrm{~min}$. $\mathrm{CL}_{\text {int }}$, intrinsic clearance; $K_{\mathrm{m}}$, the substrate concentration at half maximum velocity; $\mathrm{V}_{\max }$, maximum velocity; RLM, rat liver microsomes $(n=3)$; RJM, rat jejunum microsomes $(n=2)$; RCM, rat colon microsomes $(n=2)$; HLM, human liver microsomes $(n=50)$; HIM, human intestinal microsomes (mixture of duodenal and jejunal tissues, $n=13$ ); \pm , standard error of the calculated kinetic parameters; CI, confidence intervals.
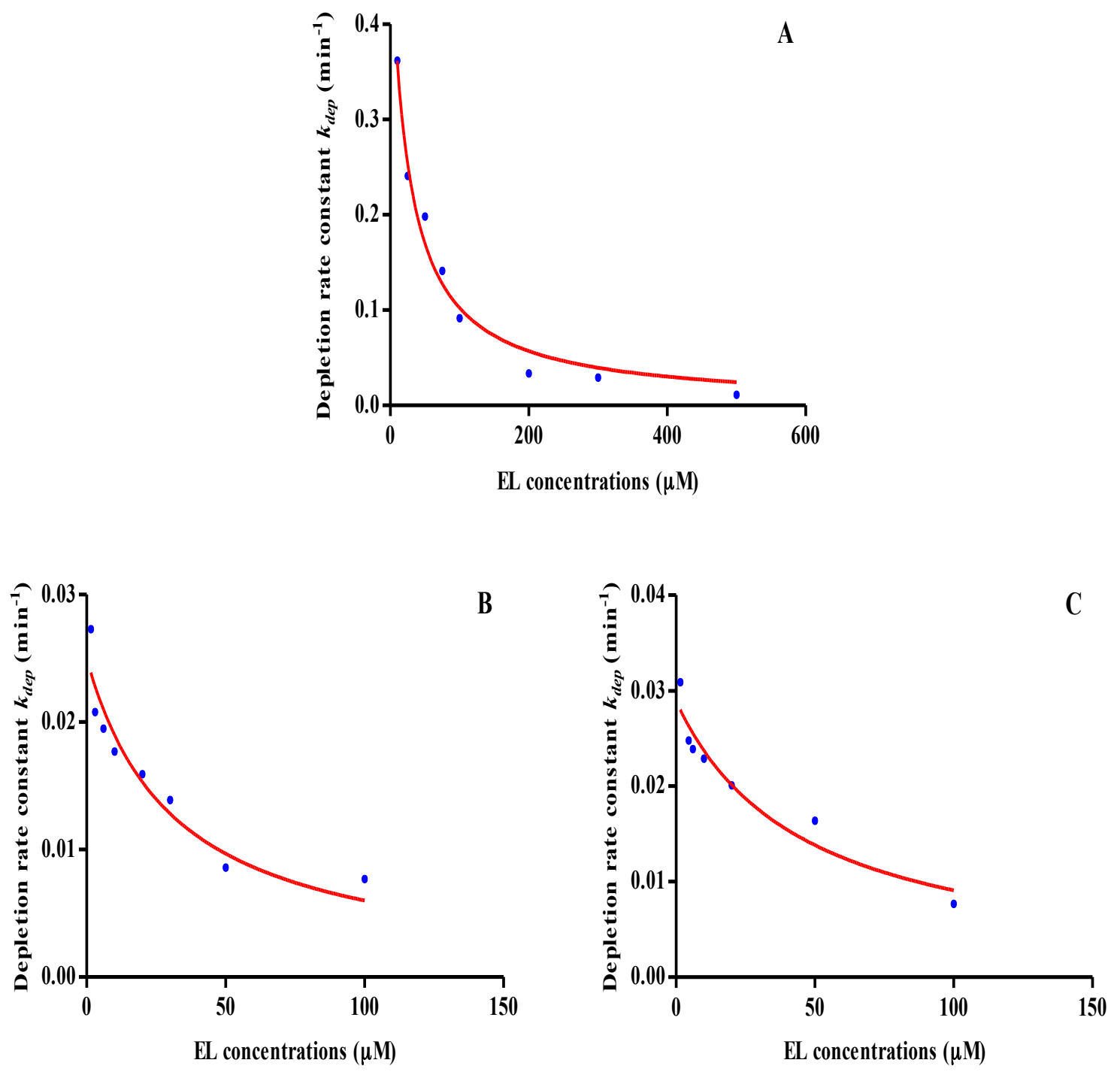

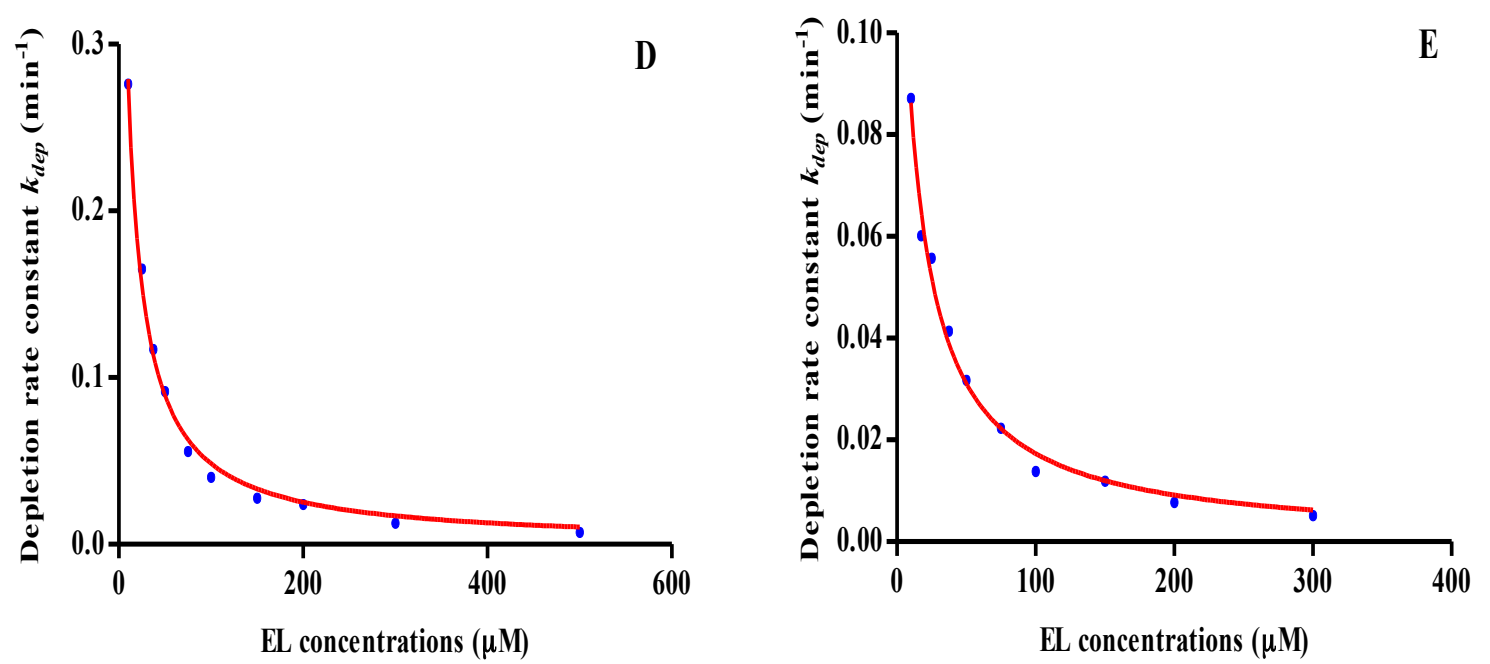

Figure 4.6 Plots of in vitro depletion rate constants $\left(k_{\mathrm{dep}}\right)\left(\mathrm{min}^{-1}\right)$ versus EL concentrations $(\mu \mathrm{M})$ for EL glucuronidation by rat liver microsomes $(\mathrm{A})$, rat jejunum microsomes $(\mathrm{B})$, rat colon microsomes (C), human liver microsomes (D), and human intestinal microsomes (E). Substrate depletion rate constant $\left(k_{\mathrm{dep}}\right)$ for each substrate concentration was equal to the slope of the linear line from a plot of ln analyte peak area percentage remaining (normalized to the initial peak area obtained at $\mathrm{t}=0$.) versus time. Refer to Appendix I for all $k_{\text {dep }}$ values for various EL concentrations for different organs from different species. 

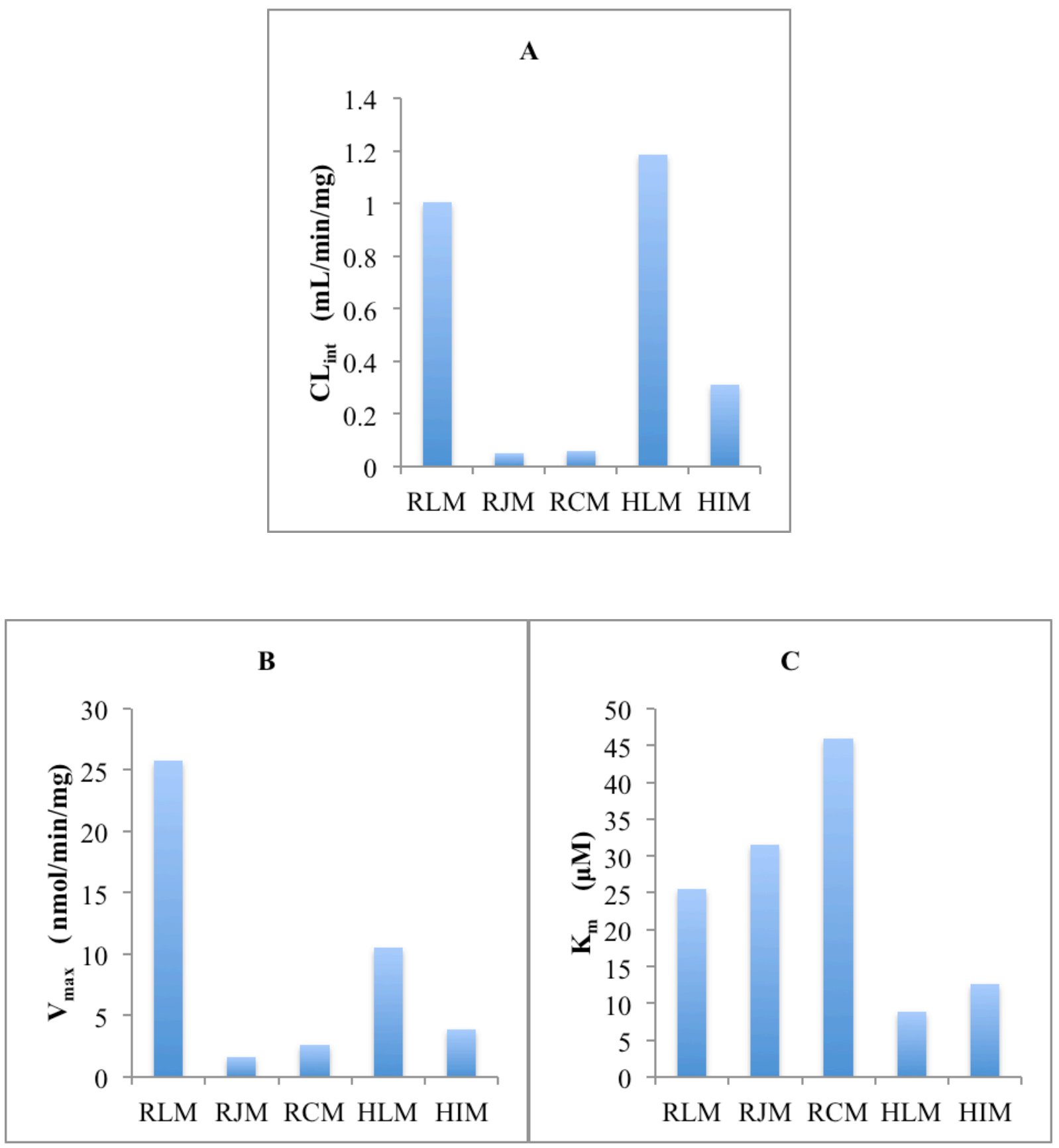

Figure 4.7 The $\mathrm{CL}_{\mathrm{int}}(\mathrm{A})$, and Michaelis-Menten constants, $\mathrm{V}_{\max }(\mathrm{B})$ and $K_{\mathrm{m}}(\mathrm{C})$, for $\mathrm{EL}$ glucuronidation by rat liver microsomes (RLM, $n=3$ ), rat jejunum microsomes (RJM, $n=2$ ), rat colon microsomes (RCM, $n=2$ ), commercial human liver microsomes (HLM, $n=50$ ) and commercial intestinal microsomes (HIM, $\mathrm{n}=13$ ) 


\section{DISCUSSION}

The aim of the project was to quantitatively make a comparison of the intestinal and hepatic glucuronidation of enterolactone (EL) derived from flaxseed lignans by the determination of intrinsic clearance $\left(\mathrm{CL}_{\mathrm{int}}\right)$ using the substrate depletion approach. Human and rat were employed to define the interspecies differences of EL glucuronidation to reduce the uncertainties of extrapolating pharmacokinetic data from rat studies to humans. The characterization of EL pharmacokinetics (e.g. metabolism) is critical to evaluate the exposure dose, susceptibility of low-dose exposure caused by first-pass metabolism, as well as drug-lignan interactions resulting from coadministration of flaxseed lignans with drugs and/or other Natural Health Products (NHPs). To accomplish this purpose, three experiments were carried out for this research project.

\subsection{EL Glucuronide Synthesis and Characterization}

My first objective was to enzymatically synthesize EL glucuronides (EL-Glu) in vitro and verify the structures by LC-MS/MS to ensure the formation of EL glucuronides, which was the foundation of enzyme kinetic studies for EL glucuronidation. Based on the literature, the suggested incubation time for EL and ED were $8 \mathrm{~h}$ and $24 \mathrm{~h}$ at $25^{\circ} \mathrm{C}$, respectively ${ }^{124}$. In order to confirm $8 \mathrm{~h}$ was sufficient to convert EL to EL-Glu, I made a comparison of the incubation reaction for EL at $8 \mathrm{~h}, 22 \mathrm{~h}$ and $24 \mathrm{~h}$, respectively, and found $22 \mathrm{~h}$ was long enough for the biotransformation of EL into EL-Glu, while the reaction was not complete at $8 \mathrm{~h}$ (data not shown). As $37^{\circ} \mathrm{C}$, representing the body temperature, is often used as the temperature for enzyme incubation, a comparison was made between 25 and $37^{\circ} \mathrm{C}$ to assess the appropriate temperature for the incubation. No significant difference existed between these two temperatures; while $25^{\circ} \mathrm{C}$ was chosen considering that this condition was easier to control.

After incubation of EL with rat liver microsomes (RLM) and purification of the generated glucuronides, a single peak at $14.1 \mathrm{~min}$ was observed by semi-preparative chromatography (4.1B). The asymmetrical peak suggested that more than one form of glucuronide was possibly produced. This was further substantiated by LC-MS/MS. Two peaks 
whose retention time were close to $9 \mathrm{~min}$, were determined by multiple reaction monitoring (MRM) mode, indicating two monoglucuronides were generated for EL incubating with RLM. As EL is not a symmetric molecule, glucuronidation of the different phenol groups would be expected to produce isomers. This result was consistent with the glucuronidation of EL in rabbit and/or rhesus monkeys liver microsomes, suggesting that EL was conjugated to either phenolic hydroxyl and was able to form two phenol $\beta$ - $O$-glucuronides ${ }^{115,124}$. Hence, the generated EL glucuronides from RLM were actually a mixture of two monoglucuronides, which can barely be separated by HPLC $^{124}$ or the LC-MS in my project. It was interesting that a single peak was detected by MRM for EL-Glu generated from rat intestinal microsomes, human liver and intestinal microsomes, implying only one or a major monoglucuronide was produced by those microsomes, which was different from the results from RLM. This might be due to the interspecies (rat versus human) as well as interorgan (liver versus intestine) differences, of which the types and content of UGT might be distinct, resulting in the varying levels of EL glucuronidation.

\subsection{Substrate Depletion Approach}

Traditionally, the kinetic behavior of an enzyme is often characterized by monitoring product formation rates at various substrate concentrations ${ }^{153}$. To fulfill this, metabolite concentrations in in vitro matrices have to be measured by analytical methods, requiring that metabolites have been definitively identified and authentic standards prepared to construct calibration curves ${ }^{154}$. However, these are not always readily and/or feasibly obtained. In my experiment, EL-Glu generated from RLM have been identified by LC-MS/MS and purified by HPLC system. Nevertheless, the purity of the fractions cannot be exactly determined to ensure highly purified EL-Glu to be used as authentic standards. Furthermore, the enzymatic synthesis method was costly both in time and money to accumulate sufficient amounts of purified EL-Glu to be used as standards because of the complex and time-consuming production process and the expensive incubation materials (EL and uridine 5'-diphosphoglucuronic acid trisodium salt). Therefore, the substrate depletion approach that monitors depletion of a substrate as a function of time was used to determine the kinetic 
parameters of the UGT. Nath and his coworkers have demonstrated that kinetic parameters obtained from the substrate depletion approach can be meaningfully compared with those obtained by measuring product formation ${ }^{153}$.

It has to be noted that the substrate depletion approach also possesses some practical limitations. One of the significant shortcomings of the substrate depletion approach is that the enzyme kinetics of formation of individual products are possibly missed, resulting in the overestimation of the individual intrinsic clearance $\left(\mathrm{CL}_{\mathrm{int}}\right)$ and/or $K_{\mathrm{m}}$ values. The obtained values represent a conglomeration of all $\mathrm{CL}_{\mathrm{int}}$ or $K_{\mathrm{m}}$ values for individual metabolic pathways $^{154}$. For EL glucuronidation, EL was incubated with microsomes that primarily contained P450 enzymes and UGT; however, only cofactor uridine 5'-diphosphoglucuronic acid trisodium salt was added into the incubation system for the conjugative reaction, which theoretically should yield only EL-Glu. This supposition was corroborated by the experimental results with HPLC analysis, which showed the appearance of a single product peak; control assays conducted without cofactor uridine 5'-diphosphoglucuronic acid trisodium salt demonstrated no product formation. Additionally, LC-MS confirmed the formation as well as the structure of EL-Glu. Therefore, the substrate depletion approach can be used to estimate the enzyme kinetic parameters for EL glucuronidation.

An important constraint when using the substrate depletion approach is the use of time points for calculation of $k_{\text {dep }}$ where no more than $10 \%$ of the substrate has been consumed; otherwise, the estimated $k_{\text {dep }}$ values will be associated with significant error, which in turn results in $K_{\mathrm{m}}$ and $V_{\max }$ values with significant error ${ }^{153}$. However, a simulation study indicated only $15 \%$ error in the estimated kinetic parameters when the data used to obtain $k_{\text {dep }}$ included time points where $50 \%$ of the substrate has been consumed ${ }^{153}$. In my study, we had to include time points with $\sim 50 \%$ (and sometimes greater) EL depletion to generate parameter estimates following the nonlinear regression analysis of the data. Hence, our calculated $k_{\text {dep }}$ values and reported $K_{\mathrm{m}}$ values have error associated with their determination (>15\%), and the calculated $\mathrm{CL}_{\text {int }}$ and $V_{\max }$ values are likely greater than their true values ${ }^{153}$. Some experimental conditions should be carefully considered to improve the substrate depletion approach in the future. As a large portion of substrate $(\sim 50 \%)$ is potentially depleted, the microsomal protein content and the incubation time should be carefully optimized to decrease the rate of substrate 
consumption, thus to minimize the errors of calculated kinetic parameters due to the over depletion of substrate.

\subsection{Interorgan Differences on EL Glucuronidation}

\subsubsection{Human Liver versus Human Intestine}

Organ and species differences in $\mathrm{CL}_{\mathrm{int}}$ and Michaelis-Menten parameters $\left(\mathrm{V}_{\max }\right.$ and $\left.K_{\mathrm{m}}\right)$ for in vitro glucuronidation of EL were investigated (Table 4.3). The results were consistent with the reports by the other, for which the microsomal UGT activities in the small intestine and /or colon were lower than that in the liver ${ }^{156}$. My study showed that all the hepatic $\mathrm{CL}_{\text {int }}$ values as well as glucuronidation rates were greater than that of the intestine, suggesting liver is predominant and more efficiently transforms EL into glucuronides as compared with the intestine. An understanding of the level of expressed UGT in the liver and intestine is necessary to further understand the relationship between hepatic and intestinal enzyme levels and glucuronidation ${ }^{157}$.

Currently, 15 human enzymes and 14 rat enzymes are known to exhibit significant conjugative activity towards endogenous and xenobiotic compounds ${ }^{119,156}$. A significant number of studies have reported the expression and localization of UGT mostly by utilizing reverse transcription-polymerase chain reaction (RT-PCR) or Northern blotting analysis ${ }^{156,158-162}$. Ten UGT are extensively expressed in human liver with large interindividual variability, including 5 UGT1 isoforms (UGT1A1, 1A3, 1A4, 1A6 and 1A9) and 5 UGT2 isoforms (UGT2B4, 2B7, 2B10, 2B15 and 2B17), and the sum of the UGT2B mRNA levels was higher than that of UGT1A mRNA levels ${ }^{160}$ (Table 5.1). An extremely high expression of UGT2B4, 2B10, 2B15, 2B7 was found, followed by some UGT1 members including UGT1A1, $1 \mathrm{~A} 6$ and 1A9 $9^{121,158-160}$.

Expression of UGTs in the human small intestine and colon is less abundant relative to the liver. Several UGT isoforms such as UGT1A1, 1A5, 1A10, 2B7, 2B15 and 2B17 are expressed, whereas UGT1A10 and 2B17 are found primarily in the intestine ${ }^{158}$. UGT1A8 is expressed in the intestine and colon but not liver ${ }^{158,163}$. Hence, the various types and considerable content of UGT1A and UGT2B families in human liver help explain why hepatic 
glucuronidation of EL was significantly larger than that in the human intestine. In addition to the UGT activity, the concentration of its significant cofactor 5'-diphosphoglucuronic acid (UDPGA) has been measured in human liver and intestinal mucosa, where it was found that the concentration of UDPGA in the liver was $279 \mathrm{nmol} / \mathrm{g}$, over 14 times higher than that in the intestinal mucosa $(19.3 \mathrm{nmol} / \mathrm{g})^{164}$. This evidence further demonstrates the overall hepatic glucuronidation activity is much greater than that in the intestine.

However, it is noteworthy the $\mathrm{CL}_{\text {int }}$ value of human intestine $\left(\mathrm{CL}_{\mathrm{int}}=0.309\right)$ was approximately one third of the value of human liver $\left(\mathrm{CL}_{\text {int }}=1.18\right)$, suggesting human intestine makes an important contribution to EL glucuronidation and may exert a significant influence on the first-pass metabolism of EL. In a study evaluating the glucuronidation of EL at $100 \mu \mathrm{M}$ with 9 expressed human UGT isoforms (UGT1A1, 1A3, 1A4, 1A6, 1A8, 1A9, 1A10, 2B7, 2B15), only human UGT1A4 and UGT1A6 failed to catalyze EL glucuronidation, while UGT2B7 and UGT1A9 showed the highest catalytic activities, followed by 1A8 and 1A10, with minor contributions from UGT1A1, $1 \mathrm{~A} 3$ and $2 \mathrm{~B} 15^{115}$. From the foregoing reports, it is known UGT1A9 and UGT2B7 are substantially expressed in the liver, whereas UGT1A8 and UGT1A10 are specific for intestine, which implies liver, as expected, is the primary organ to transform EL into glucuronides; however, the UGT isoforms such as UGT1A8 and UGT1A10 in the intestine also play a crucial role in EL glucuronidation.

The human intestinal microsomes used in my project were prepared from duodenal and jejunal tissues. The distribution of UGT is not well characterized along the small intestine in the literature. Nevertheless, based on the catalytic UGT activities examined in the small intestine and liver using 18 substrates, it was suggested that the catalytic activities were greatest in the jejunum, and the proximally located duodenum showed higher catalytic activities than the distally located ileum in 16 substrates $^{161}$. Therefore, the combination of duodenum and jejunum is able to largely represent the general UGT activity in human small intestine. Furthermore, the finding that the catalytic activities using 18 substrates in the jejunum were universally greater than those found in liver suggested that extrahepatic glucuronidation in small intestine can function to complement hepatic glucuronidation ${ }^{161}$. Although the liver has been long considered as the major organ responsible for the xenobiotic glucuronidation, the small intestine, as the first site of oral exposure, could possibly alter 
first-pass metabolism and affect bioavailability in humans. Thus, omitting the role of human intestinal glucuronidation will underestimate overall metabolic clearance ${ }^{165}$.

\subsubsection{Rat Liver versus Rat Intestine}

In rat, 7 functional members of UGT1 family and 7 UGT2 isoforms have been identified $^{119,166}$ (Table 5.2). However, the tissue distribution of rat UGT1 and UGT2 isoforms has not been thoroughly examined, especially UGT2 family. Similar to human liver, several UGT2B members are mainly expressed in rat liver including UGT2B1, 2B2, 2B3, 2B6 and $2 \mathrm{~B} 12^{167,168}$. In contrast to rat UGT2B family, of the seven members of the UGT1A family, only UGT1A1, 1A5 and 1A6 are detected at an appreciable level in rat liver ${ }^{167,169}$. In my project, the estimated hepatic $\mathrm{CL}_{\text {int }}$ was ten times higher than that of rat intestinal microsomes including both rat jejunum and colon microsomes. The substantial expression of UGT in rat liver may explain the large amount of glucuronidation by the liver. Another study that evaluated the glucuronidation of several aglycones in small intestinal microsomes of rats and compared these with glucuronidation in liver microsomes also suggested the intestinal activities were much lower than in liver, representing $5-15 \%$ of hepatic activities ${ }^{170}$, which was consistent with my results. Moreover, the UDPGA concentrations in rat liver and small intestine were 400 and $100 \mathrm{nmol} / \mathrm{g}$ tissue, respectively ${ }^{171}$, providing supportive evidence that rat liver is more competent to mediate glucuronidation than small intestine.

Multiple UGT1 family members are expressed in intestine, with UGT1A2, 1A3 and $1 \mathrm{~A} 7$ as the predominant isoforms ${ }^{167,169}$ (Table 5.2). Unlike with the UGT1 family, few UGT2B subfamily members are detected, while only UGT2B8 is the principal isoform expressed in duodenum $^{167,168}$. Quantitative evaluation of RNA levels by Northern blot revealed gradients of expression, with highest UGT1A mRNA levels found in duodenum and decreasing levels in the distal small and large intestine ${ }^{169}$. The aforementioned data suggest rat duodenum contributes more to UGT activities than other sections in intestine. However, this is somewhat against my finding that rat duodenum made the least contribution to EL glucuronidation. This discrepancy might be due to the differences in tissue collection. Alternatively, the isoforms responsible for EL glucuronidation might demonstrate higher expression in the colon. The 
$\mathrm{CL}_{\text {int }}$ for EL glucuronidation was comparable between rat jejunum and colon $(0.0500$ and 0.0578, respectively), which suggests similar UGT activity for EL glucuronidation might exist between rat jejunum and colon. 
Table 5.1 Quantitative determination for human UGT (Adapted from Ohno ${ }^{158}$ )

\begin{tabular}{|c|c|c|c|}
\hline Tissue & $\begin{array}{c}\text { Liver } \\
(\mathrm{GAPDH} 94.8 \pm 0.0)\end{array}$ & $\begin{array}{c}\text { Small intestine } \\
(\text { GAPDH } 124 \pm 0.7)\end{array}$ & $\begin{array}{c}\text { Colon } \\
(\text { GAPDH } 59.4 \pm 1.0)\end{array}$ \\
\hline $1 \mathrm{~A} 1$ & $1430 \pm 26.4$ & $582 \pm 9.7$ & $372 \pm 9.4$ \\
\hline $1 \mathrm{~A} 3$ & $131 \pm 5.4$ & $24.4 \pm 1.5$ & N.D. \\
\hline $1 \mathrm{~A} 4$ & $618 \pm 3.8$ & N.D. & N.D. \\
\hline $1 \mathrm{~A} 5$ & $8.59 \pm 2.99$ & $71.6 \pm 4.1$ & $45.6 \pm 13.4$ \\
\hline $1 \mathrm{~A} 6$ & $468 \pm 5.4$ & $94.5 \pm 2.7$ & N.D. \\
\hline $1 \mathrm{~A} 7$ & $5.07 \pm 4.00$ & $26.3 \pm 1.0$ & $25.1 \pm 4.0$ \\
\hline $1 \mathrm{~A} 8$ & N.D. & $70.0 \pm 3.5$ & $117 \pm 8.4$ \\
\hline $1 \mathrm{~A} 9$ & $1210 \pm 9.7$ & $38.3 \pm 4.4$ & $50.0 \pm 2.7$ \\
\hline $1 \mathrm{~A} 10$ & N.D. & $968 \pm 28.4$ & $754 \pm 32.4$ \\
\hline $2 \mathrm{~B} 4$ & $37,900 \pm 711$ & N.D. & N.D. \\
\hline $2 \mathrm{~B} 7$ & $4220 \pm 12.7$ & $1930 \pm 7.4$ & $2210 \pm 30.3$ \\
\hline $2 \mathrm{~B} 10$ & $3380 \pm 92.7$ & N.D. & N.D. \\
\hline $2 \mathrm{~B} 11$ & N.D. & N.D. & N.D. \\
\hline 2B15 & $18,500 \pm 285$ & $738 \pm 168.5$ & $543 \pm 132.8$ \\
\hline $2 \mathrm{~B} 17$ & $197 \pm 12.6$ & $2680 \pm 2.8$ & $3960 \pm 20.2$ \\
\hline
\end{tabular}

Values shown are copy number $\times 10^{4}$ normalized with GAPDH (glyceraldehyde-3-phosphate dehydrogenase), mean \pm S.D.

Table 5.2 The expression of UGT in rat tissues at the detection levels ${ }^{167,169}$

\begin{tabular}{|l|l|l|}
\hline \multicolumn{1}{|c|}{ Tissue } & \multicolumn{1}{|c|}{ UGT1 } & \multicolumn{1}{|c|}{ UGT2 } \\
\hline Rat & $\begin{array}{l}\text { 1A1, 1A2, 1A3,1A5, 1A6, 1A7, } \\
1 \mathrm{~A} 8\end{array}$ & $\begin{array}{l}\text { 2A1, 2B1, 2B2, 2B3, 2B6, } \\
2 \mathrm{~B} 8,2 \mathrm{~B} 12\end{array}$ \\
\hline Rat liver & $1 \mathrm{~A} 1,1 \mathrm{~A} 5,1 \mathrm{~A} 6,1 \mathrm{~A} 8$ & $2 \mathrm{~B} 1,2 \mathrm{~B} 2,2 \mathrm{~B} 3,2 \mathrm{~B} 6,2 \mathrm{~B} 12$ \\
\hline Rat small intestine & $1 \mathrm{~A} 1,1 \mathrm{~A} 2,1 \mathrm{~A} 3,1 \mathrm{~A} 6,1 \mathrm{~A} 7$ & $2 \mathrm{~B} 3,2 \mathrm{~B} 8,2 \mathrm{~B} 12$ \\
\hline Rat colon & $1 \mathrm{~A} 1,1 \mathrm{~A} 2,1 \mathrm{~A} 3,1 \mathrm{~A} 6,1 \mathrm{~A} 7$ & $2 \mathrm{~B} 12$ \\
\hline
\end{tabular}




\subsection{Interspecies Differences on EL Glucuronidation}

Interspecies differences in EL glucuronidation were also observed in my study. Rat is frequently used as an experimental model to evaluate the pharmacokinetic properties of xenobiotics including lignans, the data of which is significant to explore species differences to reduce the uncertainties related to data extrapolation from animals to humans. In my study, quantitative determination of EL glucuronidation was performed to determine the suitability of rat as a model to evaluate EL metabolism in humans. My evaluations suggested that some species discrepancies existed, especially with regard to intestinal metabolism. Based on the data, $\mathrm{CL}_{\text {int }}$ value of human liver was only slightly higher than the value from rat liver, while the human intestinal $\mathrm{CL}_{\text {int }}$ was much greater than the value either from RJM or RCM. Though the commercial human intestinal microsomes were a mixture of duodenum and jejunum tissues, the relatively great differences in $\mathrm{CL}_{\text {int }}$ values imply human intestinal glucuronidation of EL was higher than that of rat intestine. These data suggests the human intestine might have a greater influence on the first-pass metabolism of EL, thereby decreasing EL's oral bioavailability. Therefore, rat can be used as a reasonable model to evaluate the hepatic glucuronidation of EL occurring in humans, while the extent of intestinal glucuronidation of EL in humans might be underestimated when experiments are carried out on rats.

\subsection{The Significance of $K_{\mathrm{m}}$ Value}

$K_{\mathrm{m}}$ is defined as the substrate concentration that yields half the theoretical maximal velocity at infinite substrate concentration. The pharmacokinetic parameters $K_{\mathrm{m}}, \mathrm{V}_{\max }$ and $\mathrm{CL}_{\text {int }}$, play an important role in drug discovery and development, which aids to understand the importance of xenobiotic metabolizing enzymes in the clearance of new drug entity, thereby enabling the assessment of potential drug-drug interactions, the influence of genetic polymorphisms, and also possible non-linear dose-exposure ${ }^{172}$. Non-linear dose exposure is not solely dependent on but is significantly related to $K_{\mathrm{m}}{ }^{173} . K_{\mathrm{m}}$ reflects the potential of a drug's ability to saturate, or partially saturate, a specific enzyme in metabolism. The lower the $K_{\mathrm{m}}$ value, the greater the affinity of the substrate to the enzyme, which might cause saturation of a metabolic clearance pathway at low drug concentrations depending upon enzyme capacity 
(i.e. $\left.\mathrm{V}_{\max }\right)^{173}$. This can be problematic with non-linear dose-exposure relationships, particular for drugs with low-therapeutic indices. Examples include cyclophosphamide, 5-fluorouracil, fluvoxamine, indinavir, and nefazodone, where a small increase in dose might cause toxicity as drug concentration in vivo will increase to a greater extent than expected ${ }^{154}$. The $K_{\mathrm{m}}$ values for HLM and HIM are 8.91 and $12.6 \mu \mathrm{M}$, respectively, which are at least 2-fold smaller when compared with the values from RLM or rat intestinal microsomes (Table 4.3), implying EL has a higher affinity towards UGT enzymes in human liver and intestine than in rat tissues. Alternatively, this could reflect simple experimental variation. The higher affinity of EL towards UGT might result in drug-drug interactions with glucuronide drugs such as morphine. More than one UGT enzyme such as UGT2B7, 1A1, and 1A8 are suggested to metabolize morphine into the more potent and active metabolite morphine-6-glucuronide and inactive morphine-3-glucuronide, with a $K_{\mathrm{m}}$ value over $60 \mu \mathrm{M}^{174-176}$. Thus, the higher affinity of EL towards UGT might cause saturation of UGT enzymes or competitive inhibition, resulting in the decreased analgesic effects of morphine. Consequently, lignans or the initial dose of lignans should be regarded cautiously when coadministered with glucuronide drugs where the principal metabolic pathway involves UGT enzymes. Given only a two-fold difference in $K_{\mathrm{m}}$ values, the rat might be a suitable model to estimate the interactions between EL and a coadministered compound. 


\section{CONCLUSIONS AND FUTURE WORK}

The purpose for this study is to provide an estimate of the relative contribution of the intestine and liver to presystemic glucuronidation of enterolactone (EL), thereby understanding the critical information regarding the oral absorption properties of EL. To fulfill this goal, in vitro enzyme kinetic studies using intestinal and liver microsomes from human and rat with determination of intrinsic clearance $\left(\mathrm{CL}_{\mathrm{int}}\right)$ using the substrate depletion approach was conducted to allow the comparison of differences of EL glucuronidation between the two first-pass metabolism organs and among these two species. Prior to carrying out the enzyme kinetic studies, it was necessary to make sure EL glucuronidation was able to occur in both human and rat intestinal and liver microsomes. In addition, an analytical method required validation to quantify EL in microsomes. Therefore, three objectives were established for my project.

For my first objective, EL glucuronide (EL-Glu) was enzymatically generated by incubating EL with intestinal and liver microsomes from both human and rat. The formation and molecular identity of EL-Glu were substantiated by liquid chromatography-mass spectrometry (LC-MS). MS analysis suggested that two monoglucuronides were formed for EL incubated with rat liver microsomes (RLM), while only a single monoglucuronide was found for rat intestinal, human liver and intestinal microsomes.

For my second objective, a simple and reliable HPLC method, which was developed in our lab, was employed for the quantitative determination of EL in RLM. The specificity was determined in the matrices including rat and human intestinal and liver microsomes. The results showed no interfering endogenous substances existed. The intra- and interday precision and accuracy for EL were $\leq 10 \%$, suggesting this HPLC method was precise and accurate to quantify EL in rat and human liver and intestinal microsomes.

For my final objective and the aim for my project, the in vitro $\mathrm{CL}_{\text {int }}$ values of $\mathrm{EL}$ glucuronidation were examined using RLM, rat jejunum microsomes (RJM) and rat colon microsomes (RCM) extracted by myself in the lab and commercial sources of human liver microsomes (HLM) and human intestinal microsomes (HIM) by the substrate depletion approach. Overall, EL hepatic glucuronidation was greater than intestinal glucuronidation in 
both human and rat, indicating liver is the predominant organ responsible for EL glucuronidation as expected. The in vitro $\mathrm{CL}_{\text {int }}$ value of HLM was slightly higher than that of RLM. $\mathrm{CL}_{\text {int }}$ value of human intestine was one third of human liver, suggesting the human intestine is likely to make a great contribution to the first-pass glucuronidation of EL, which might largely reduce the oral bioavailability of EL. The hepatic $\mathrm{CL}_{\text {int }}$ value of rat was twenty times higher than that of the RJM or RCM, respectively. RJM and RCM gave comparable $\mathrm{CL}_{\text {int }}$ values but were much lower than that of HIM. In brief, these results indicate rat can be used as models to evaluate hepatic glucuronidation of EL in humans, while experiments on rats may underestimate the extent of intestinal glucuronidation of EL that occurs in humans.

In future, the UGT isoforms and their abundance responsible for EL glucuronidation in human liver and intestine need to be identified. This will provide insight into the importance of specific UGT enzymes to the pharmacokinetic properties of EL. Furthermore, the identification of specific UGT isoforms responsible for EL glucuronidation will potentially help avoid drug-drug interactions when lignans are coadministrated with drugs or other Natural Health Products (NPHs) that are predominantly metabolized by the same types of UGT. Pharmacokinetic information of EL-Glu needs to be investigated, as EL-Glu is the principal forms in human plasma. The determination of biological properties of EL-Glu will assist us to understand the lignan forms mediating the health beneficial effects. Additional pharmacokinetic information of EL needs to be thoroughly examined so that lignans can be consumed safely and efficiently. 


\section{REFERENCES}

1. Government of Canada HC, Health Products and Food Branch, Natural Health Products Directorate. Natural Health Products-Drugs and Health Products. 2012; http://www.hc-sc.gc.ca/dhp-mps/prodnatur/index-eng.php/.

2. Baseline Natural Health Products Survey Among Consumers. 2005; http://site.ebrary.com.cyber.usask.ca/lib/usask/docDetail.action?docID=10089399.

3. Government of Canada HC, Natural Health Products Directorate. Overview of the Natural Health Products Regulations Guidance Document; 2012; http://www.hc-sc.gc.ca/dhp-mps/prodnatur/legislation/docs/regula-regle_over-apercu-e ng.php.

4. Government of Canada HC, Health Products and Food Branch, Natural Health Products Directorate. Evidence for Safety and Efficacy of Finished Natural Health Products. 2006;

http://www.hc-sc.gc.ca/dhp-mps/prodnatur/legislation/docs/efe-paie-eng.php.

5. Government of Canada HC. Draft: Pathway for licensing natural health products making modern health claims. 2012;

http://www.hc-sc.gc.ca/dhp-mps/consultation/natur/consult_modern-eng.php.

6. Government of Canada HC, Health Products and Food Branch, Natural Health Products Directorate. About Natural Health Product Regulations in Canada. 2012; http://www.hc-sc.gc.ca/dhp-mps/prodnatur/about-apropos/index-eng.php.

7. He SM, Chan E, Zhou SF. ADME properties of herbal medicines in humans: evidence, challenges and strategies. Curr Pharm Des. 2011;17(4):357-407.

8. Jordan SA, Cunningham DG, Marles RJ. Assessment of herbal medicinal products: challenges, and opportunities to increase the knowledge base for safety assessment. Toxicol Appl Pharmacol. 2010;243(2):198-216.

9. Izzo AA, Ernst E. Interactions between herbal medicines and prescribed drugs: an updated systematic review. Drugs. 2009;69(13):1777-1798.

10. Singh YN, Singh NN. Therapeutic potential of kava in the treatment of anxiety disorders. CNS Drugs. 2002;16(11):731-743.

11. Anke J, Ramzan I. Pharmacokinetic and pharmacodynamic drug interactions with Kava (Piper methysticum Forst. f.). J Ethnopharmacol. 2004;93(2-3):153-160.

12. Alkharfy KM, Frye RF. Effect of valerian, valerian/hops extracts, and valerenic acid on glucuronidation in vitro. Xenobiotica. 2007;37(2):113-123. 
13. Zhang YS, Tu YY, Gao XC, et al. Strong Inhibition of Celastrol Towards UDP-Glucuronosyl Transferase (UGT) 1A6 and 2B7 Indicating Potential Risk of UGT-Based Herb-Drug Interaction. Molecules. 2012;17(6):6832-6839.

14. Mohamed ME, Frye RF. Inhibitory effects of commonly used herbal extracts on UDP-glucuronosyltransferase 1A4, 1A6, and 1A9 enzyme activities. Drug Metab Dispos. 2011;39(9):1522-1528.

15. Abdel-Rahman A, Anyangwe N, Carlacci L, et al. The safety and regulation of natural products used as foods and food ingredients. Toxicol Sci. 2011;123(2):333-348.

16. Murty M. Postmarket surveillance of natural health products in Canada: clinical and federal regulatory perspectives. Can J Physiol Pharmacol. 2007;85(9):952-955.

17. Shan JJ, Rodgers K, Lai CT, Sutherland SK. Challenges in natural health product research: The importance of standardization. Proc West Pharmacol Soc. 2007; 50:24-30.

18. Canadian Grain Commission. Nutritional profile of no. 1. Canada Western flaxseed and of yellow flaxseed samples. Canadian Grain Commission, Winnipeg, MB. 2001.

19. Daun JK, Barthet VJ, Chornick TL, Duguid S. Structure, Composition, and Variety Development of Flaxseed. Flaxseed in Human Nutrition, Second Edition. 2003.

20. Bloedon LT, Balikai S, Chittams J, et al. Flaxseed and cardiovascular risk factors: results from a double blind, randomized, controlled clinical trial. $J$ Am Coll Nutr. 2008;27(1):65-74.

21. Patade A, Devareddy L, Lucas EA, Korlagunta K, Daggy BP, Arjmandi BH. Flaxseed reduces total and LDL cholesterol concentrations in Native American postmenopausal women. $J$ Womens Health (Larchmt). 2008;17(3):355-366.

22. Demark-Wahnefried W, Polascik TJ, George SL, et al. Flaxseed supplementation (not dietary fat restriction) reduces prostate cancer proliferation rates in men presurgery. Cancer Epidemiol Biomarkers Prev. 2008;17(12):3577-3587.

23. Thompson LU, Chen JM, Li T, Strasser-Weippl K, Goss PE. Dietary flaxseed alters tumor biological markers in postmenopausal breast cancer. Clin Cancer Res. 2005; 11(10):3828-3835.

24. Government of Canada HC, Health Products and Food Branch, Natural Health Products Directorate, Bureau of Product Review and Assessment. Licensed Natural Health Products Database (LNHPD). [meeting material]. 2012; http://webprod3.hc-sc.gc.ca/lnhpd-bdpsnh/search-recherche.do?lang=eng.

25. Oomah BD, Mazza G. Bioactive Components of Flaxseed: Occurrence and Health Benefits. Phytochemicals and Phytopharmaceuticals. 1999;106-121. 
26. Prasad K. Reduction of serum cholesterol and hypercholesterolemic atherosclerosis in rabbits by secoisolariciresinol diglucoside isolated from flaxseed. Circulation. 1999; 99(10):1355-1362.

27. Prasad K. Hypocholesterolemic and antiatherosclerotic effect of flax lignan complex isolated from flaxseed. Atherosclerosis. 2005;179(2):269-275.

28. Zhang W, Wang X, Liu Y, et al. Dietary flaxseed lignan extract lowers plasma cholesterol and glucose concentrations in hypercholesterolaemic subjects. Br J Nutr. 2008;99(6):1301-1309.

29. Jenab M, Thompson LU. The influence of flaxseed and lignans on colon carcinogenesis and beta-glucuronidase activity. Carcinogenesis. 1996;17(6):1343-48.

30. Bergman Jungestrom M, Thompson LU, Dabrosin C. Flaxseed and its lignans inhibit estradiol-induced growth, angiogenesis, and secretion of vascular endothelial growth factor in human breast cancer xenografts in vivo. Clin Cancer Res. 2007; 13(3):1061-1067

31. Pietinen P, Stumpf K, Mannisto S, Kataja V, Uusitupa M, Adlercreutz H. Serum enterolactone and risk of breast cancer: a case-control study in eastern Finland. Cancer Epidemiol Biomarkers Prev. 2001;10(4):339-344.

32. Prasad K. Hydroxyl radical-scavenging property of secoisolariciresinol diglucoside (SDG) isolated from flax-seed. Mol Cell Biochem. 1997;168(1-2):117-123.

33. Kitts DD, Yuan YV, Wijewickreme AN, Thompson LU. Antioxidant activity of the flaxseed lignan secoisolariciresinol diglycoside and its mammalian lignan metabolites enterodiol and enterolactone. Mol Cell Biochem. 1999;202(1-2):91-100.

34. Velasquez MT, Bhathena SJ, Ranich T, et al. Dietary flaxseed meal reduces proteinuria and ameliorates nephropathy in an animal model of type II diabetes mellitus. Kidney Int. 2003;64(6):2100-2107.

35. Prasad K. Secoisolariciresinol diglucoside from flaxseed delays the development of type 2 diabetes in Zucker rat. J Lab Clin Med. 2001;138(1):32-39.

36. Moss GP. Nomenclature of Lignans and Neolignans (IUPAC Recommendations 2000). Pure and Applied Chemistry. 2012;72(8):1493-1523.

37. Meagher LP, Beecher GR. Assessment of data on the lignan content of foods. J Food Comp Anal. 2000;13(6):935-947.

38. Mazur W, Adlercreutz H. Overview of naturally occurring endocrine-active substances in the human diet in relation to human health. Nutrition. 2000;16(7-8):654-658. 
39. Milder IE, Arts IC, van de Putte B, Venema DP, Hollman PC. Lignan contents of Dutch plant foods: a database including lariciresinol, pinoresinol, secoisolariciresinol and matairesinol. Br J Nutr. 2005;93(3):393-402.

40. Thompson LU, Boucher BA, Liu Z, Cotterchio M, Kreiger N. Phytoestrogen content of foods consumed in Canada, including isoflavones, lignans, and coumestan. Nutr Cancer. 2006;54(2):184-201.

41. Axelson M, Sjövall J, Gustafsson BE, Setchell KD. Origin of lignans in mammals and identification of a precursor from plants. Nature. 1982;298(5875):659-660.

42. Heinonen $S$, Nurmi $T$, Liukkonen $K$, Poutanen $K$, Wähälä $K$, Deyama T, Nishibe $S$, Adlercreutz H. In Vitro Metabolism of Plant Lignans: New Precursors of Mammalian Lignans Enterolactone and Enterodiol. J Agric Food Chem. 2001;49(7);3178-86.

43. Tan KP, Chen J, Ward WE, Thompson LU. Mammary gland morphogenesis is enhanced by exposure to flaxseed or its major lignan during suckling in rats. Exp Biol Med (Maywood). 2004;229(2):147-157.

44. Serraino M, Thompson LU. Flaxseed supplementation and early markers of colon carcinogenesis. Cancer Lett. 1992;63(2):159-165.

45. Hallund J, Tetens I, Bugel S, Tholstrup T, Bruun JM. The effect of a lignan complex isolated from flaxseed on inflammation markers in healthy postmenopausal women. Nutr Metab Cardiovasc Dis. 2008;18(7):497-502.

46. Saarinen NM, Abrahamsson A, Dabrosin C. Estrogen-induced angiogenic factors derived from stromal and cancer cells are differently regulated by enterolactone and genistein in human breast cancer in vivo. Int J Cancer. 2010;127(3):737-745.

47. Hu C, Yuan YV, Kitts DD. Antioxidant activities of the flaxseed lignan secoisolariciresinol diglucoside, its aglycone secoisolariciresinol and the mammalian lignans enterodiol and enterolactone in vitro. Food Chem Toxicol. 2007; 45(11):2219-2227.

48. Ingram D, Sanders K, Kolybaba M, Lopez D. Case-control study of phyto-oestrogens and breast cancer. Lancet. 1997;350(9083):990-994.

49. Dai Q, Franke AA, Jin F, et al. Urinary excretion of phytoestrogens and risk of breast cancer among Chinese women in Shanghai. Cancer Epidemiol Biomarkers Prev. 2002;11(9):815-821.

50. Kuijsten A, Arts IC, Hollman PC, van't Veer P, Kampman E. Plasma enterolignans are associated with lower colorectal adenoma risk. Cancer Epidemiol Biomarkers Prev. 2006;15(6):1132-1136. 
51. Hedelin M, Klint A, Chang ET, et al. Dietary phytoestrogen, serum enterolactone and risk of prostate cancer: the cancer prostate Sweden study (Sweden). Cancer Causes Control. 2006;17(2):169-180.

52. Wang LQ. Mammalian phytoestrogens: enterodiol and enterolactone. J Chromatogr B Analyt Technol Biomed Life Sci. 2002;777(1-2):289-309.

53. Linseisen J, Piller R, Hermann S, Chang-Claude J. Dietary phytoestrogen intake and premenopausal breast cancer risk in a German case-control study. Int J Cancer. 2004;110(2):284-290.

54. McCann SE, Muti P, Vito D, Edge SB, Trevisan M, Freudenheim JL. Dietary lignan intakes and risk of pre- and postmenopausal breast cancer. Int J Cancer. 2004; 111(3):440-443.

55. Cotterchio M, Boucher BA, Kreiger N, Mills CA, Thompson LU. Dietary phytoestrogen intake--lignans and isoflavones--and breast cancer risk (Canada). Cancer Causes Control. 2008;19(3):259-272.

56. Suzuki R, Rylander-Rudqvist T, Saji S, Bergkvist L, Adlercreutz H, Wolk A. Dietary lignans and postmenopausal breast cancer risk by oestrogen receptor status: a prospective cohort study of Swedish women. Br J Cancer. 2008;98(3):636-640.

57. McCann SE, Moysich KB, Freudenheim JL, Ambrosone CB, Shields PG. The risk of breast cancer associated with dietary lignans differs by CYP17 genotype in women. $J$ Nutr. 2002;132(10):3036-3041.

58. dos Santos Silva I, Mangtani P, McCormack V, Bhakta D, McMichael AJ, Sevak L. Phyto-oestrogen intake and breast cancer risk in South Asian women in England: findings from a population-based case-control study. Cancer Causes Control. 2004;15(8):805-818.

59. Hedelin M, Lof M, Olsson M, Adlercreutz H, Sandin S, Weiderpass E. Dietary phytoestrogens are not associated with risk of overall breast cancer but diets rich in coumestrol are inversely associated with risk of estrogen receptor and progesterone receptor negative breast tumors in Swedish women. J Nutr. 2008;138(5):938-945.

60. Keinan-Boker L, van Der Schouw YT, Grobbee DE, Peeters PH. Dietary phytoestrogens and breast cancer risk. Am J Clin Nutr. 2004;79(2):282-288.

61. Touillaud MS, Thiebaut AC, Niravong M, Boutron-Ruault MC, Clavel-Chapelon F. No association between dietary phytoestrogens and risk of premenopausal breast cancer in a French cohort study. Cancer Epidemiol Biomarkers Prev.

2006;15(12):2574-2576. 
62. Kilkkinen A, Virtamo J, Vartiainen E, et al. Serum enterolactone concentration is not associated with breast cancer risk in a nested case-control study. Int J Cancer. 2004;108(2):277-280.

63. den Tonkelaar I, Keinan-Boker L, Veer PV, et al. Urinary phytoestrogens and postmenopausal breast cancer risk. Cancer Epidemiol Biomarkers Prev. 2001; 10(3):223-228.

64. Hulten K, Winkvist A, Lenner P, Johansson R, Adlercreutz H, Hallmans G. An incident case-referent study on plasma enterolactone and breast cancer risk. Eur J Nutr. 2002;41(4):168-176.

65. Rickard SE, Yuan YV, Chen J, Thompson LU. Dose effects of flaxseed and its lignan on N-methyl-N-nitrosourea-induced mammary tumorigenesis in rats. Nutr Cancer. 1999;35(1):50-57.

66. Chen J, Tan KP, Ward WE, Thompson LU. Exposure to flaxseed or its purified lignan during suckling inhibits chemically induced rat mammary tumorigenesis. Exp Biol Med (Maywood). 2003;228(8):951-958.

67. Prasad K. Regression of hypercholesterolemic atherosclerosis in rabbits by secoisolariciresinol diglucoside isolated from flaxseed. Atherosclerosis. 2008; 197(1):34-42.

68. Buck K, Zaineddin AK, Vrieling A, Linseisen J, Chang-Claude J. Meta-analyses of lignans and enterolignans in relation to breast cancer risk. Am J Clin Nutr. 2010; 92(1):141-153.

69. Landete JM. Plant and mammalian lignans: A review of source, intake, metabolism, intestinal bacteria and health. Food Research International. 2012;46(1):410-424.

70. Ames BN, Shigenaga MK, Hagen TM. Oxidants, antioxidants, and the degenerative diseases of aging. Proc Natl Acad Sci U S A. 1993;90(17):7915-7922.

71. Hosseinian FS, Muir AD, Westcott ND, Krol ES. Antioxidant capacity of flaxseed lignans in two model systems. J Am Oil Chem Soc. 2006;83(10):835-840.

72. Hosseinian FS, Muir AD, Westcott ND, Krol ES. AAPH-mediated antioxidant reactions of secoisolariciresinol and SDG. Org Biomol Chem. 2007;5(4):644-654.

73. Prasad K. Antioxidant Activity of Secoisolariciresinol Diglucoside-derived Metabolites, Secoisolariciresinol, Enterodiol, and Enterolactone. Int J Angiol. 2000; 9(4):220-225.

74. O'Malley BW. Mechanisms of action of steroid hormones. N Engl J Med. 1971; 284(7):370-377. 
75. Korach KS, Migliaccio S, Davis VL. Estrogens. In: Munson PL (ed). Principles of Pharmacology-Basic Concepts and Clinical Applications. New York:Chapman and Hall. 1995;809-826.

76. Deroo BJ, Korach KS. Estrogen receptors and human disease. J Clin Invest. 2006; 116(3):561-570.

77. Mueller SO, Korach KS. Estrogen receptors and endocrine diseases: lessons from estrogen receptor knockout mice. Curr Opin Pharmacol. 2001;1(6):613-619.

78. Nilsson S, Gustafsson JA. Estrogen receptor transcription and transactivation: Basic aspects of estrogen action. Breast Cancer Res. 2000;2(5):360-366.

79. Mueller SO, Korach KS. Mechanisms of Estrogen Receptor-Mediated Agonistic and Antagonistic Effects. The Handbook of Environmental Chemistry. 2001;3L:545.

80. Ososki AL, Kennelly EJ. Phytoestrogens: a review of the present state of research. Phytother Res. 2003;17(8):845-869.

81. Setchell KD. Phytoestrogens: the biochemistry, physiology, and implications for human health of soy isoflavones. Am J Clin Nutr. 1998;68(6 Suppl):1333S-1346S.

82. Carreau C, Flouriot G, Bennetau-Pelissero C, Potier M. Enterodiol and enterolactone, two major diet-derived polyphenol metabolites have different impact on ERalpha transcriptional activation in human breast cancer cells. $J$ Steroid Biochem Mol Biol. 2008;110(1-2):176-185.

83. Sathyamoorthy N, Wang TT, Phang JM. Stimulation of $\mathrm{pS} 2$ expression by diet-derived compounds. Cancer Res. 1994;54(4):957-961.

84. Mousavi Y, Adlercreutz H. Enterolactone and estradiol inhibit each other's proliferative effect on MCF-7 breast cancer cells in culture. J Steroid Biochem Mol Biol. 1992;41(3-8):615-619.

85. Wang C, Kurzer MS. Phytoestrogen concentration determines effects on DNA synthesis in human breast cancer cells. Nutr Cancer. 1997;28(3):236-247.

86. Westphal U. Steroid-protein interactions II. Monogr Endocrinol. 1986;27:1-603.

87. Martin ME, Haourigui M, Pelissero C, Benassayag C, Nunez EA. Interactions between phytoestrogens and human sex steroid binding protein. Life Sci. 1996;58(5):429-436.

88. Adlercreutz H, Hockerstedt $\mathrm{K}$, Bannwart C, et al. Effect of dietary components, including lignans and phytoestrogens, on enterohepatic circulation and liver metabolism of estrogens and on sex hormone binding globulin (SHBG). J Steroid Biochem. 1987;27(4-6):1135-1144. 
89. Adlercreutz H, Mousavi Y, Clark J, et al. Dietary phytoestrogens and cancer: in vitro and in vivo studies. J Steroid Biochem Mol Biol. 1992;41(3-8):331-337.

90. Kellis JT, Jr., Vickery LE. Purification and characterization of human placental aromatase cytochrome P-450. J Biol Chem. 1987;262(9):4413-4420.

91. Wang C, Makela T, Hase T, Adlercreutz H, Kurzer MS. Lignans and flavonoids inhibit aromatase enzyme in human preadipocytes. J Steroid Biochem Mol Biol. 1994; 50(3-4):205-212.

92. Adlercreutz $\mathrm{H}$, Bannwart $\mathrm{C}$, Wahala $\mathrm{K}$, et al. Inhibition of human aromatase by mammalian lignans and isoflavonoid phytoestrogens. J Steroid Biochem Mol Biol. 1993;44(2):147-153.

93. Evans BA, Griffiths K, Morton MS. Inhibition of 5 alpha-reductase in genital skin fibroblasts and prostate tissue by dietary lignans and isoflavonoids. J Endocrinol. 1995;147(2):295-302.

94. Brooks JD, Thompson LU. Mammalian lignans and genistein decrease the activities of aromatase and 17beta-hydroxysteroid dehydrogenase in MCF-7 cells. J Steroid Biochem Mol Biol. 2005;94(5):461-467.

95. Kamal-Eldin A, Peerlkamp N, Johnsson P, et al. An oligomer from flaxseed composed of secoisolariciresinoldiglucoside and 3-hydroxy-3-methyl glutaric acid residues. Phytochemistry. 2001;58(4):587-590.

96. Li X, Yuan JP, Xu SP, Wang JH, Liu X. Separation and determination of secoisolariciresinol diglucoside oligomers and their hydrolysates in the flaxseed extract by high-performance liquid chromatography. J Chromatogr A. 2008;1185(2):223-232.

97. Jansen GH, Arts IC, Nielen MW, Muller M, Hollman PC, Keijer J. Uptake and metabolism of enterolactone and enterodiol by human colon epithelial cells. Arch Biochem Biophys. 2005;435(1):74-82.

98. Axelson M, Setchell KD. The excretion of lignans in rats -- evidence for an intestinal bacterial source for this new group of compounds. FEBS Lett. 1981;123(2):337-342.

99. Bannwart C, Adlercreutz H, Wahala K, Brunow G, Hase T. Detection and identification of the plant lignans lariciresinol, isolariciresinol and secoisolariciresinol in human urine. Clin Chim Acta. 1989;180(3):293-301.

100. Rickard SE, Thompson LU. Urinary composition and postprandial blood changes in $\mathrm{H}$-secoisolariciresinol diglycoside (SDG) metabolites in rats do not differ between acute and chronic SDG treatments. J Nutr. 2000;130(9):2299-2305. 
101. Wang LQ, Meselhy MR, Li Y, Qin GW, Hattori M. Human intestinal bacteria capable of transforming secoisolariciresinol diglucoside to mammalian lignans, enterodiol and enterolactone. Chem Pharm Bull (Tokyo). 2000;48(11):1606-1610.

102. Mukker JK, Kotlyarova V, Singh RS, Alcorn J. HPLC method with fluorescence detection for the quantitative determination of flaxseed lignans. J Chromatogr B Analyt Technol Biomed Life Sci. 2010;878(30):3076-3082.

103. Kuijsten A, Arts IC, Vree TB, Hollman PC. Pharmacokinetics of enterolignans in healthy men and women consuming a single dose of secoisolariciresinol diglucoside. $J$ Nutr. 2005;135(4):795-801.

104. Mazur WM, Uehara M, Wahala K, Adlercreutz H. Phyto-oestrogen content of berries, and plasma concentrations and urinary excretion of enterolactone after a single strawberry-meal in human subjects. Br J Nutr. 2000;83(4):381-387.

105. Borriello SP, Setchell KD, Axelson M, Lawson AM. Production and metabolism of lignans by the human faecal flora. J Appl Bacteriol. 1985;58(1):37-43.

106. Clavel $\mathrm{T}$, Henderson $\mathrm{G}$, Alpert $\mathrm{CA}$, et al. Intestinal bacterial communities that produce active estrogen-like compounds enterodiol and enterolactone in humans. Appl Environ Microbiol. 2005;71(10):6077-6085.

107. Kilkkinen A, Pietinen P, Klaukka T, Virtamo J, Korhonen P, Adlercreutz H. Use of oral antimicrobials decreases serum enterolactone concentration. Am J Epidemiol. 2002;155(5):472-477.

108. Clavel T, Henderson G, Engst W, Dore J, Blaut M. Phylogeny of human intestinal bacteria that activate the dietary lignan secoisolariciresinol diglucoside. FEMS Microbiol Ecol. 2006;55(3):471-478.

109. Glitso LV, Mazur WM, Adlercreutz H, et al. Intestinal metabolism of rye lignans in pigs. Br J Nutr. 2000;84(4):429-437.

110. Eeckhaut E, Struijs K, Possemiers S, Vincken JP, Keukeleire DD, Verstraete W. Metabolism of the lignan macromolecule into enterolignans in the gastrointestinal lumen as determined in the simulator of the human intestinal microbial ecosystem. $J$ Agric Food Chem. 2008;56(12):4806-4812.

111. Pettersson $D$, Aman $P$, Knudsen KE, et al. Intake of rye bread ileostomists increases ileal excretion of fiber polysaccharide components and organic acids but does not increase plasma or urine lignans and isoflavonoids. J Nutr. 1996;126(6):1594-1600.

112. Laerke HN, Mortensen MA, Hedemann MS, Bach Knudsen KE, Penalvo JL, Adlercreutz H. Quantitative aspects of the metabolism of lignans in pigs fed fibre-enriched rye and wheat bread. Br J Nutr. 2009;102(7):985-994. 
113. Clavel T, Lippman R, Gavini F, Dore J, Blaut M. Clostridium saccharogumia sp. nov. and Lactonifactor longoviformis gen. nov., sp. nov., two novel human faecal bacteria involved in the conversion of the dietary phytoestrogen secoisolariciresinol diglucoside. Syst Appl Microbiol. 2007;30(1):16-26.

114. Niemeyer HB, Honig D, Lange-Bohmer A, Jacobs E, Kulling SE, Metzler M. Oxidative metabolites of the mammalian lignans enterodiol and enterolactone in rat bile and urine. J Agric Food Chem. 2000;48(7):2910-2919.

115. Dean B, Chang S, Doss GA, King C, Thomas PE. Glucuronidation, oxidative metabolism, and bioactivation of enterolactone in rhesus monkeys. Arch Biochem Biophys. 2004;429(2):244-251.

116. Jacobs E, Metzler M. Oxidative metabolism of the mammalian lignans enterolactone and enterodiol by rat, pig, and human liver microsomes. J Agric Food Chem. 1999; 47(3):1071-1077.

117. Jacobs E, Kulling SE, Metzler M. Novel metabolites of the mammalian lignans enterolactone and enterodiol in human urine. J Steroid Biochem Mol Biol. 1999; 68(5-6):211-218.

118. Muir AD, Westcott ND. Mammalian metabolism of flax lignans. Flax. 2003(11).

119. Tukey RH, Strassburg CP. Human UDP-glucuronosyltransferases: metabolism, expression, and disease. Annu Rev Pharmacol Toxicol. 2000;40:581-616.

120. Ritter JK. Roles of glucuronidation and UDP-glucuronosyltransferases in xenobiotic bioactivation reactions. Chem Biol Interact. 2000;129(1-2):171-193.

121. Jancova $P$, Anzenbacher $P$, Anzenbacherova E. Phase II drug metabolizing enzymes. Biomed Pap Med Fac Univ Palacky Olomouc Czech Repub. 2010;154(2):103-116.

122. Axelson M, Setchell KD. Conjugation of lignans in human urine. FEBS Lett. 1980; 122(1):49-53.

123. Adlercreutz H, Fotsis $T$, Lampe $J$, et al. Quantitative determination of lignans and isoflavonoids in plasma of omnivorous and vegetarian women by isotope dilution gas chromatography-mass spectrometry. Scand J Clin Lab Invest Suppl. 1993;215:5-18.

124. Brunner G, Tegtmeier F, Kirk DN, Wynn S, Setchell KD. Enzymatic synthesis and chromatographic purification of lignan glucuronides. Biomed Chromatogr. 1986; 1(2):89-92.

125. Adlercreutz $\mathrm{H}$, van der Wildt J, Kinzel J, et al. Lignan and isoflavonoid conjugates in human urine. J Steroid Biochem Mol Biol. 1995;52(1):97-103. 
126. Adlercreutz H, Borriello SP, Lawson AM. Formation of lignans by intestinal microflora. Falk Symposium 31, Colonic Carcinogenesis. MTP Press Ltd., Lancaster. 1982;93-97.

127. Murray T, Kang J, Astheimer L, Price WE. Tissue distribution of lignans in rats in response to diet, dose-response, and competition with isoflavones. J Agric Food Chem. 2007;55(12):4907-4912.

128. Dehennin L, Reiffsteck A, Jondet M, Thibier M. Identification and quantitative estimation of a lignan in human and bovine semen. J Reprod Fertil. 1982; 66(1):305309.

129. Boccardo F, Lunardi GL, Petti AR, Rubagotti A. Enterolactone in breast cyst fluid: correlation with EGF and breast cancer risk. Breast Cancer Res Treat. 2003; 79(1):17-23.

130. Rickard SE, Thompson LU. Chronic exposure to secoisolariciresinol diglycoside alters lignan disposition in rats. J Nutr. 1998;128(3):615-623.

131. Saarinen NM, Thompson LU. Prolonged administration of secoisolariciresinol diglycoside increases lignan excretion and alters lignan tissue distribution in adult male and female rats. Br J Nutr. 2010;104(6):833-841.

132. Schottner M, Gansser D, Spiteller G. Interaction of lignans with human sex hormone binding globulin (SHBG). Z Naturforsch C. 1997;52(11-12):834-843.

133. Schottner M, Spiteller G. Lignans Interfering with $5 \alpha$-Dihydrotestosterone Binding to Human Sex Hormone-Binding Globulin. [brief-report]. 1998; http://pubs.acs.org/doi/abs/10.1021/np9701743.

134. Adlercreutz H, Fotsis $T$, Bannwart $C$, et al. Determination of urinary lignans and phytoestrogen metabolites, potential antiestrogens and anticarcinogens, in urine of women on various habitual diets. J Steroid Biochem. 1986;25(5B):791-797.

135. Adlercreutz H, Fotsis $\mathrm{T}$, Heikkinen R, et al. Diet and urinary excretion of lignans in female subjects. Med Biol. 1981;59(4):259-261.

136. Rickard SE, Orcheson LJ, Seidl MM, Luyengi L, Fong HH, Thompson LU. Dose-dependent production of mammalian lignans in rats and in vitro from the purified precursor secoisolariciresinol diglycoside in flaxseed. $J$ Nutr. 1996;126(8):2012-2019.

137. Hutchins AM, Martini MC, Olson BA, Thomas W, Slavin JL. Flaxseed influences urinary lignan excretion in a dose-dependent manner in postmenopausal women. Cancer Epidemiol Biomarkers Prev. 2000;9(10):1113-1118. 
138. Kurzer MS, Lampe JW, Martini MC, Adlercreutz H. Fecal lignan and isoflavonoid excretion in premenopausal women consuming flaxseed powder. Cancer Epidemiol Biomarkers Prev. 1995;4(4):353-358.

139. Bach Knudsen KE, Serena A, Kjaer AK, et al. Rye bread in the diet of pigs enhances the formation of enterolactone and increases its levels in plasma, urine and feces. $J$ Nutr. 2003;133(5):1368-1375.

140. Horner NK, Kristal AR, Prunty J, Skor HE, Potter JD, Lampe JW. Dietary determinants of plasma enterolactone. Cancer Epidemiol Biomarkers Prev. 2002;11(1):121-126.

141. Kilkkinen A, Stumpf K, Pietinen P, Valsta LM, Tapanainen H, Adlercreutz H. Determinants of serum enterolactone concentration. Am J Clin Nutr. 2001; 73(6):1094-1100.

142. Johnsen NF, Hausner $\mathrm{H}$, Olsen A, et al. Intake of whole grains and vegetables determines the plasma enterolactone concentration of Danish women. J Nutr. 2004;134(10):2691-2697.

143. Possemiers S, Bolca S, Eeckhaut E, Depypere H, Verstraete W. Metabolism of isoflavones, lignans and prenylflavonoids by intestinal bacteria: producer phenotyping and relation with intestinal community. FEMS Microbiol Ecol. 2007;61(2):372-383.

144. Brownlee IA. The physiological roles of dietary fibre. Food Hydrocolloid. 2011; 25(2):238-250.

145. Kilkkinen A, Valsta LM, Virtamo J, Stumpf K, Adlercreutz H, Pietinen P. Intake of lignans is associated with serum enterolactone concentration in Finnish men and women. J Nutr. 2003;133(6):1830-1833.

146. Pelkonen O, Turpeinen M. In vitro-in vivo extrapolation of hepatic clearance: biological tools, scaling factors, model assumptions and correct concentrations. Xenobiotica. 2007;37(10-11):1066-1089.

147. Donato MT, Castell JV. Strategies and molecular probes to investigate the role of cytochrome P450 in drug metabolism: focus on in vitro studies. Clin Pharmacokinet. 2003;42(2):153-178.

148. Brandon EF, Raap CD, Meijerman I, Beijnen JH, Schellens JH. An update on in vitro test methods in human hepatic drug biotransformation research: pros and cons. Toxicol Appl Pharmacol. 2003;189(3):233-246.

149. Zhang ZY, Wong YN. Enzyme kinetics for clinically relevant CYP inhibition. Curr Drug Metab. 2005;6(3):241-257.

150. Iannaccone PM, Jacob HJ. Rats! Dis Model Mech. 2009;2(5-6):206-210. 
151. Weiser MM. Intestinal epithelial cell surface membrane glycoprotein synthesis. I. An indicator of cellular differentiation. J Biol Chem. 1973;248(7):2536-2541.

152. Kosaka K, Sakai N, Endo Y, et al. Impact of intestinal glucuronidation on the pharmacokinetics of raloxifene. Drug Metab Dispos. 2011;39(9):1495-1502.

153. Nath A, Atkins WM. A theoretical validation of the substrate depletion approach to determining kinetic parameters. Drug Metab Dispos. 2006;34(9):1433-1435.

154. Obach RS, Reed-Hagen AE. Measurement of Michaelis constants for cytochrome P450-mediated biotransformation reactions using a substrate depletion approach. Drug Metab Dispos. 2002;30(7):831-837.

155. Komura H, Iwaki M. Pharmacokinetics and metabolism of metoprolol and propranolol in the female DA and female Wistar rat: the female DA rat is not always an animal model for poor metabolizers of CYP2D6. J Pharm Sci. 2005;94(2):397-408.

156. Strassburg CP, Nguyen N, Manns MP, Tukey RH. UDP-glucuronosyltransferase activity in human liver and colon. Gastroenterology. 1999;116(1):149-160.

157. Bock KW. Functions and transcriptional regulation of adult human hepatic UDP-glucuronosyl-transferases (UGTs): mechanisms responsible for interindividual variation of UGT levels. Biochem Pharmacol. 2010;80(6):771-777.

158. Ohno S, Nakajin S. Determination of mRNA expression of human UDP-glucuronosyltransferases and application for localization in various human tissues by real-time reverse transcriptase-polymerase chain reaction. Drug Metab Dispos. 2009;37(1):32-40.

159. Nakamura A, Nakajima M, Yamanaka H, Fujiwara R, Yokoi T. Expression of UGT1A and UGT2B mRNA in human normal tissues and various cell lines. Drug Metab Dispos. 2008;36(8):1461-1464.

160. Izukawa $T$, Nakajima $M$, Fujiwara $R$, et al. Quantitative analysis of UDP-glucuronosyltransferase (UGT) 1A and UGT2B expression levels in human livers. Drug Metab Dispos. 2009;37(8):1759-1768.

161. Strassburg CP, Kneip S, Topp J, et al. Polymorphic gene regulation and interindividual variation of UDP-glucuronosyltransferase activity in human small intestine. J Biol Chem. 2000;275(46):36164-36171.

162. Ikushiro S, Emi Y, Kato Y, Yamada S, Sakaki T. Monospecific antipeptide antibodies against human hepatic UDP-glucuronosyltransferase 1A subfamily (UGT1A) isoforms. Drug Metab Pharmacokinet. 2006;21(1):70-74. 
163. Gregory PA, Lewinsky RH, Gardner-Stephen DA, Mackenzie PI. Regulation of UDP glucuronosyltransferases in the gastrointestinal tract. Toxicol Appl Pharmacol. 2004;199(3):354-363.

164. Cappiello M, Giuliani L, Pacifici GM. Distribution of UDP-glucuronosyltransferase and its endogenous substrate uridine 5'-diphosphoglucuronic acid in human tissues. Eur J Clin Pharmacol. 1991;41(4):345-350.

165. Cubitt HE, Houston JB, Galetin A. Relative importance of intestinal and hepatic glucuronidation-impact on the prediction of drug clearance. Pharm Res. 2009; 26(5):1073-1083.

166. Emi Y, Ikushiro S, Iyanagi T. Drug-responsive and tissue-specific alternative expression of multiple first exons in rat UDP-glucuronosyltransferase family 1 (UGT1) gene complex. J Biochem. 1995;117(2):392-399.

167. Shelby MK, Cherrington NJ, Vansell NR, Klaassen CD. Tissue mRNA expression of the rat UDP-glucuronosyltransferase gene family. Drug Metab Dispos. 2003; 31(3):326-333.

168. Mackenzie PI. Rat liver UDP-glucuronosyltransferase. Identification of cDNAs encoding two enzymes which glucuronidate testosterone, dihydrotestosterone, and beta-estradiol. J Biol Chem. 1987;262(20):9744-9749.

169. Grams B, Harms A, Braun S, Strassburg CP, Manns MP, Obermayer-Straub P. Distribution and inducibility by 3 -methylcholanthrene of family 1 UDP-glucuronosyltransferases in the rat gastrointestinal tract. Arch Biochem Biophys. 2000;377(2):255-265.

170. Vargas M, Franklin MR. Intestinal UDP-glucuronosyltransferase activities in rat and rabbit. Xenobiotica. 1997;27(5):413-421.

171. Goon D, Klaassen CD. Effects of microsomal enzyme inducers upon UDP-glucuronic acid concentration and UDP-glucuronosyltransferase activity in the rat intestine and liver. Toxicol Appl Pharmacol. 1992;115(2):253-260.

172. Youdim K, Dodia R. Comparison between recombinant P450s and human liver microsomes in the determination of cytochrome P450 Michaelis-Menten constants. Xenobiotica. 2010;40(4):235-244.

173. Ludden TM. Nonlinear pharmacokinetics: clinical Implications. Clin Pharmacokinet. 1991;20(6):429-446.

174. Coughtrie MW, Ask B, Rane A, Burchell B, Hume R. The enantioselective glucuronidation of morphine in rats and humans. Evidence for the involvement of more than one UDP-glucuronosyltransferase isoenzyme. Biochem Pharmacol. 1989;38(19):3273-3280. 
175. Stone AN, Mackenzie PI, Galetin A, Houston JB, Miners JO. Isoform selectivity and kinetics of morphine 3- and 6-glucuronidation by human udp-glucuronosyltransferases: evidence for atypical glucuronidation kinetics by UGT2B7. Drug Metab Dispos. 2003;31(9):1086-1089.

176. Ohno S, Kawana K, Nakajin S. Contribution of UDP-glucuronosyltransferase $1 \mathrm{~A} 1$ and 1A8 to morphine-6-glucuronidation and its kinetic properties. Drug Metab Dispos. 2008;36(4):688-694. 


\section{APPENDIX I}

Table A.1 The natural log percent remaining for EL at multiple time points when a low EL concentration was incubated with rat liver microsomes

\begin{tabular}{|c|c|c|c|c|}
\hline \multicolumn{5}{|c|}{ Rat liver microsomes } \\
\hline $25 \mu \mathrm{M}$ & & $\begin{array}{l}\text { The value of peak area } \\
\text { ratios at multiple time } \\
\text { points was normalized to } \\
\mathrm{t}=0\end{array}$ & Percent & Ln percent \\
\hline $\begin{array}{l}\text { Peak area ratios of analyte/IS } \\
\text { at time } \mathrm{t}=0 \mathrm{~min}\end{array}$ & 1.32 & 1 & 100 & 4.60 \\
\hline $\begin{array}{l}\text { Peak area ratios of analyte/IS } \\
\text { at time } \mathrm{t}=2.5 \mathrm{~min}\end{array}$ & 0.72 & 0.541 & 54.1 & 3.99 \\
\hline $\begin{array}{l}\text { Peak area ratios of analyte/IS } \\
\text { at time } \mathrm{t}=5 \mathrm{~min}\end{array}$ & 0.47 & 0.355 & 35.5 & 3.57 \\
\hline $\begin{array}{l}\text { Peak area ratios of analyte/IS } \\
\text { at time } \mathrm{t}=7.5 \mathrm{~min}\end{array}$ & 0.28 & 0.209 & 20.9 & 3.04 \\
\hline $\begin{array}{l}\text { Peak area ratios of analyte/IS } \\
\text { at time } \mathrm{t}=10 \mathrm{~min}\end{array}$ & 0.14 & 0.109 & 10.9 & 2.39 \\
\hline $\begin{array}{l}\text { Peak area ratios of analyte/IS } \\
\text { at time } \mathrm{t}=12.5 \mathrm{~min}\end{array}$ & 0.07 & 0.054 & 5.4 & 1.69 \\
\hline $\begin{array}{l}\text { Peak area ratios of analyte/IS } \\
\text { at time } \mathrm{t}=15 \mathrm{~min}\end{array}$ & 0.03 & 0.025 & 2.5 & 0.92 \\
\hline
\end{tabular}

A low EL concentration $(25 \mu \mathrm{M})$ was chosen as an example to show the process of generating ln percent remaining, then to further plot a linear line to obtain $k_{\text {dep }}$ value for each EL concentration.

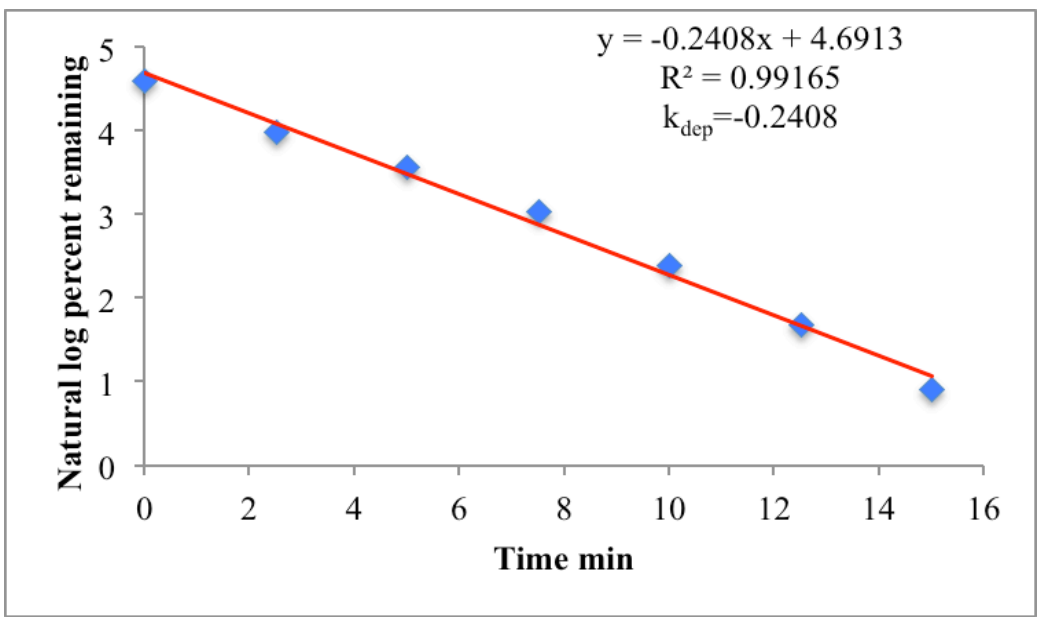

Figure A.1 A plot of natural log percent remaining versus time 
Table A.2 The natural log percent remaining for EL at multiple time points when a high EL concentration was incubated with rat liver microsomes

\begin{tabular}{|c|c|c|c|c|}
\hline \multicolumn{5}{|c|}{ Rat liver microsomes } \\
\hline $300 \mu \mathrm{M}$ & & $\begin{array}{l}\text { The value of peak area } \\
\text { ratios at multiple time } \\
\text { points is normalized to } \\
\mathrm{t}=0\end{array}$ & Percent & Ln percent \\
\hline $\begin{array}{l}\text { Peak area ratios of analyte/IS } \\
\text { at time } \mathrm{t}=0 \mathrm{~min}\end{array}$ & 14.97 & 1 & 100 & 4.60 \\
\hline $\begin{array}{l}\text { Peak area ratios of analyte/IS } \\
\text { at time } \mathrm{t}=5 \mathrm{~min}\end{array}$ & 11.80 & 0.788 & 78.8 & 4.37 \\
\hline $\begin{array}{l}\text { Peak area ratios of analyte/IS } \\
\text { at time } \mathrm{t}=10 \mathrm{~min}\end{array}$ & 10.70 & 0.715 & 71.5 & 4.27 \\
\hline $\begin{array}{l}\text { Peak area ratios of analyte/IS } \\
\text { at time } \mathrm{t}=15 \mathrm{~min}\end{array}$ & 9.62 & 0.643 & 64.3 & 4.16 \\
\hline $\begin{array}{l}\text { Peak area ratios of analyte/IS } \\
\text { at time } \mathrm{t}=20 \mathrm{~min}\end{array}$ & 8.49 & 0.567 & 56.7 & 4.04 \\
\hline $\begin{array}{l}\text { Peak area ratios of analyte/IS } \\
\text { at time } \mathrm{t}=25 \mathrm{~min}\end{array}$ & 7.21 & 0.482 & 48.2 & 3.88 \\
\hline $\begin{array}{l}\text { Peak area ratios of analyte/IS } \\
\text { at time } \mathrm{t}=30 \mathrm{~min}\end{array}$ & 5.77 & 0.385 & 38.5 & 3.65 \\
\hline
\end{tabular}

A high EL concentration $(300 \mu \mathrm{M})$ was chosen as an example to show the process of generating $\ln$ percent remaining, then to further plot a linear line to obtain $k_{\text {dep }}$ value for each EL concentration.

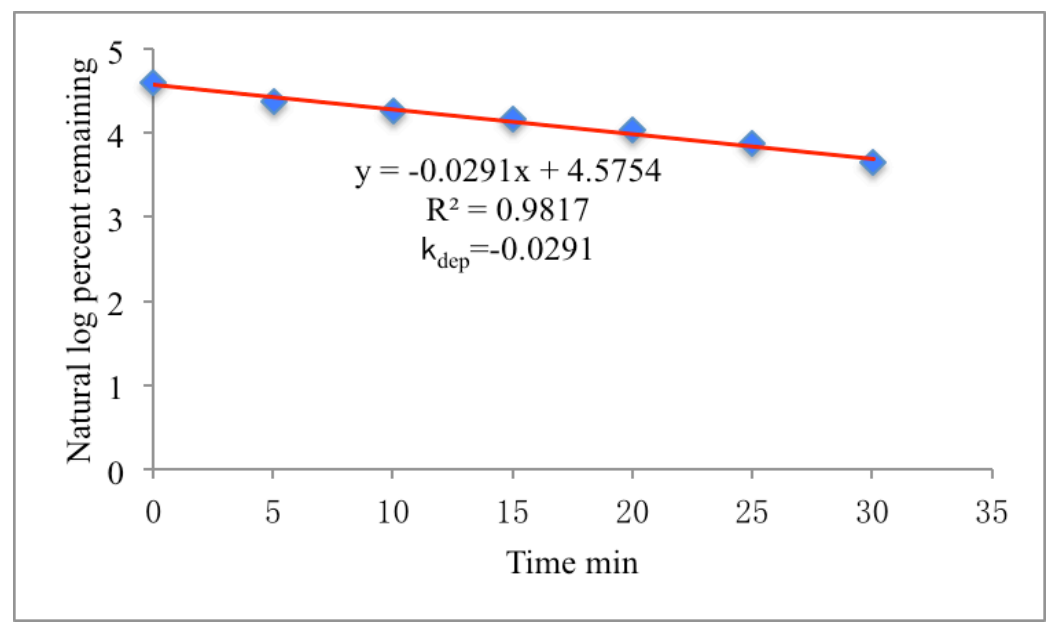

Figure A.2 A plot of natural log percent remaining versus time 
Table A.3 Various EL concentrations $(10-500 \mu \mathrm{M})$ versus percent remaining for rat liver microsomal kinetic study

\begin{tabular}{ccc}
\hline EL Concentration $(\mu \mathrm{M})$ & Final percent remaining & $k_{\mathrm{dep}}\left(\mathrm{min}^{-1}\right)$ \\
10 & 2.6 & 0.362 \\
25 & 2.5 & 0.241 \\
50 & 1.7 & 0.198 \\
75 & 5.5 & 0.141 \\
100 & 5.4 & 0.0916 \\
200 & 35.6 & 0.0335 \\
300 & 38.5 & 0.0291 \\
500 & 70.8 & 0.0110 \\
\hline
\end{tabular}

Table A.4 Various EL concentrations $(1.5-100 \mu \mathrm{M})$ versus percent remaining for rat jejunum microsomal kinetic study

\begin{tabular}{ccc}
\hline EL Concentration $(\mu \mathrm{M})$ & Final percent remaining & $k_{\mathrm{dep}}\left(\mathrm{min}^{-1}\right)$ \\
1.5 & 42.6 & 0.0273 \\
3 & 58.2 & 0.0208 \\
6 & 55.3 & 0.0195 \\
10 & 57.7 & 0.0177 \\
20 & 60.1 & 0.0159 \\
30 & 64.5 & 0.0139 \\
50 & 78.1 & 0.0086 \\
100 & 78.3 & 0.0077 \\
\hline
\end{tabular}

Table A.5 Various EL concentrations $(1.5-100 \mu \mathrm{M})$ versus percent remaining for rat colon microsomal kinetic study

\begin{tabular}{ccc}
\hline EL Concentration $(\mu \mathrm{M})$ & Final percent remaining & $k_{\mathrm{dep}}\left(\mathrm{min}^{-1}\right)$ \\
1.5 & 45.8 & 0.0309 \\
4.5 & 45 & 0.0248 \\
6 & 44.7 & 0.0239 \\
10 & 48.4 & 0.0229 \\
20 & 52.5 & 0.0201 \\
50 & 58 & 0.0164 \\
100 & 77.9 & 0.0077 \\
\hline
\end{tabular}


Table A.6 Various EL concentrations $(10-500 \mu \mathrm{M})$ versus percent remaining for human liver microsomal kinetic study

\begin{tabular}{ccc}
\hline EL Concentration $(\mu \mathrm{M})$ & Final percent remaining & $k_{\text {dep }}\left(\mathrm{min}^{-1}\right)$ \\
10 & 1.5 & 0.276 \\
25 & 1.3 & 0.165 \\
37.5 & 2.3 & 0.117 \\
50 & 5 & 0.0917 \\
75 & 16.2 & 0.0556 \\
100 & 27.5 & 0.0402 \\
150 & 42.3 & 0.0277 \\
200 & 45.3 & 0.0238 \\
300 & 66.5 & 0.0127 \\
500 & 80.7 & 0.0070 \\
\hline
\end{tabular}

Table A.7 Various EL concentrations $(10-300 \mu \mathrm{M})$ versus percent remaining for human intestinal microsomal kinetic study

\begin{tabular}{ccc}
\hline EL Concentration $(\mu \mathrm{M})$ & Final percent remaining & $k_{\mathrm{dep}}\left(\mathrm{min}^{-1}\right)$ \\
10 & 6.4 & 0.0871 \\
17.5 & 14.6 & 0.0601 \\
25 & 17.4 & 0.0557 \\
37.5 & 25.4 & 0.0414 \\
50 & 35.9 & 0.0317 \\
75 & 50.5 & 0.0223 \\
100 & 63.8 & 0.0138 \\
150 & 68.6 & 0.0119 \\
200 & 79 & 0.0077 \\
300 & 84.9 & 0.0051 \\
\hline
\end{tabular}

